\title{
Coincidence point and common fixed point theorems in the product spaces of mixed-monotonically complete quasi-ordered metric spaces and their applications to the systems of integral equations and ordinary differential equations
}

Hsien-Chung Wu*

"Correspondence:

hcwu@nknucc.nknu.edu.tw

Department of Mathematics,

National Kaohsiung Normal

University, Kaohsiung, 802, Taiwan

\begin{abstract}
The main purpose of this paper is to study the coincidence point and common fixed point theorems in the product spaces of mixed-monotonically complete quasi-ordered metric spaces based on some new types of contractive inequalities. In order to investigate the existence and chain-uniqueness of solutions for the systems of integral equations and ordinary differential equations, we shall also study the fixed point theorems for the functions having mixed monotone property or comparable property in the product space of quasi-ordered metric space.
\end{abstract}

MSC: $47 \mathrm{H} 10 ; 54 \mathrm{H} 25$

Keywords: function of contractive factor; coincidence point; common fixed points; chain-uniqueness; system of integral equations; system of ordinary differential equations

\section{Introduction}

The existence of coincidence point has been studied in [1-4] and the references therein. Also, the existence of common fixed point has been studied in [5-15] and the references therein. In this paper, we shall introduce the concepts of mixed-monotonically complete quasi-ordered metric space and monotonically complete quasi-ordered metric space. Based on this completeness, we shall establish some new coincidence point and common fixed point theorems in the product spaces of mixed-monotonically complete quasi-ordered metric spaces in which the fixed points of functions having mixed monotone property or mixed comparable property that are defined in the product space of quasi-ordered metric space can be subsequently obtained. We shall also present the interesting applications to the existence and chain-uniqueness of solutions for the systems of integral equations and ordinary differential equations according to the fixed points of functions having mixed monotone property.

O2014 Wu; licensee Springer. This is an Open Access article distributed under the terms of the Creative Commons Attribution License (http://creativecommons.org/licenses/by/2.0), which permits unrestricted use, distribution, and reproduction in any medium, provided the original work is properly cited. 
In Section 2, we shall derive the coincidence point theorems in the product space of mixed-monotonically complete quasi-ordered metric space. Also, in Section 3, the coincidence point theorems in the product space of monotonically complete quasi-ordered metric space will be studied. On the other hand, in Section 4, we shall study the fixed point theorems for the functions having mixed monotone property in the product space of monotonically complete quasi-ordered metric space. Also, in Section 5, the fixed point theorems for the functions having mixed comparable property in the product space of mixed-monotonically complete quasi-ordered metric space will be derived. In Section 6, we shall present the interesting application to investigate the existence and chainuniqueness of solutions for the system of integral equations. Finally, in Section 7, we shall also present the interesting application to investigate the existence and chain-uniqueness of solutions for the system of ordinary differential equations.

\section{Coincidence point theorems in the mixed-monotonically complete quasi-ordered metric space}

Let $X$ be a nonempty set. We consider the product set

$$
X^{m}=\underbrace{X \times \cdots \times X}_{m \text { times }} .
$$

The element of $X^{m}$ is represented by the vectorial notation $\mathbf{x}=\left(x^{(1)}, \ldots, x^{(m)}\right)$, where $x^{(i)} \in X$ for $i=1, \ldots, m$. We also consider the function $\mathbf{F}: X^{m} \rightarrow X^{m}$ defined by

$$
\mathbf{F}(\mathbf{x})=\left(F_{1}(\mathbf{x}), F_{2}(\mathbf{x}), \ldots, F_{m}(\mathbf{x})\right)
$$

where $F_{k}: X^{m} \rightarrow X$ for all $k=1,2, \ldots, m$. The vectorial element $\hat{\mathbf{x}}=\left(\hat{x}^{(1)}, \hat{x}^{(2)}, \ldots, \hat{x}^{(m)}\right) \in X^{m}$ is a fixed point of $\mathbf{F}$ if and only if $\mathbf{F}(\hat{\mathbf{x}})=\hat{\mathbf{x}}$; that is,

$$
F_{k}\left(\hat{x}^{(1)}, \hat{x}^{(2)}, \ldots, \hat{x}^{(m)}\right)=\hat{x}^{(k)}
$$

for all $k=1,2, \ldots, m$.

Definition 2.1 Let $X$ be a nonempty set. Consider the functions $\mathbf{F}: X^{m} \rightarrow X^{m}$ and $\mathbf{f}$ : $X^{m} \rightarrow X^{m}$ by $\mathbf{F}=\left(F_{1}, F_{2}, \ldots, F_{k}\right)$ and $\mathbf{f}=\left(f_{1}, f_{2}, \ldots, f_{k}\right)$, where $F_{k}: X^{m} \rightarrow X$ and $f_{k}: X^{m} \rightarrow X$ for $k=1,2, \ldots, m$.

- The element $\hat{\mathbf{x}} \in X^{m}$ is a coincidence point of $\mathbf{F}$ and $\mathbf{f}$ if and only if $\mathbf{F}(\hat{\mathbf{x}})=\mathbf{f}(\hat{\mathbf{x}})$, i.e., $F_{k}(\hat{\mathbf{x}})=f_{k}(\hat{\mathbf{x}})$ for all $k=1,2, \ldots, m$.

- The element $\hat{\mathbf{x}}$ is a common fixed point of $\mathbf{F}$ and $\mathbf{f}$ if and only if $\mathbf{F}(\hat{\mathbf{x}})=\mathbf{f}(\hat{\mathbf{x}})=\hat{\mathbf{x}}$, i.e., $F_{k}(\hat{\mathbf{x}})=f_{k}(\hat{\mathbf{x}})=\hat{x}^{(k)}$ for all $k=1,2, \ldots, m$.

- The functions $\mathbf{F}$ and $\mathbf{f}$ are said to be commutative if and only if $\mathbf{f}(\mathbf{F}(\mathbf{x}))=\mathbf{F}(\mathbf{f}(\mathbf{x}))$ for all $\mathbf{x} \in X^{m}$.

Let ' $\leq$ ' be a binary relation defined on $X$. We say that the binary relation ' $\preceq$ ' is a quasiorder (pre-order or pseudo-order) if and only if it is reflexive and transitive. In this case, $(X, \preceq)$ is called a quasi-ordered set.

For any $\mathbf{x}, \mathbf{y} \in X^{m}$, we say that $\mathbf{x}$ and $\mathbf{y}$ are $\preceq$-mixed comparable if and only if, for each $k=1, \ldots, m$, one has either $x^{(k)} \preceq y^{(k)}$ or $y^{(k)} \preceq x^{(k)}$. Let $I$ be a subset of $\{1,2, \ldots, m\}$ and 
$J=\{1,2, \ldots, m\} \backslash I$. In this case, we say that $I$ and $J$ are the disjoint pair of $\{1,2, \ldots, m\}$. We can define a binary relation on $X^{m}$ as follows:

$$
\mathbf{x} \preccurlyeq I \mathbf{y} \quad \text { if and only if } \quad x^{(k)} \preceq y^{(k)} \quad \text { for } k \in I \quad \text { and } \quad y^{(k)} \preceq x^{(k)} \quad \text { for } k \in J .
$$

It is obvious that $\left(X^{m}, \preccurlyeq_{I}\right)$ is a quasi-ordered set that depends on $I$. We also have

$$
\mathbf{x} \preccurlyeq_{I} \mathbf{y} \quad \text { if and only if } \mathbf{y} \preccurlyeq_{J} \mathbf{x} \text {. }
$$

We need to mention that $I$ or $J$ is allowed to be an empty set.

Remark 2.2 For any $\mathbf{x}, \mathbf{y} \in X^{m}$, we have the following observations.

(a) If $\mathbf{x} \preccurlyeq_{I} \mathbf{y}$ for some disjoint pair $I$ and $J$ of $\{1, \ldots, m\}$, then $\mathbf{x}$ and $\mathbf{y}$ are $\preceq$-mixed comparable.

(b) If $\mathbf{x}$ and $\mathbf{y}$ are $\preceq$-mixed comparable, then there exists a disjoint pair $I$ and $J$ of $\{1, \ldots, m\}$ such that $\mathbf{x} \preccurlyeq_{I} \mathbf{y}$.

Definition 2.3 Let $I$ and $J$ be a disjoint pair of $\{1,2, \ldots, m\}$. Given a quasi-ordered set $(X, \preceq)$, we consider the quasi-ordered set $\left(X^{m}, \preccurlyeq_{I}\right)$ defined in (1).

- The sequence $\left\{x_{n}\right\}_{n \in \mathbb{N}}$ in $X$ is said to be a mixed $\preceq$-monotone sequence if and only if $x_{n} \preceq x_{n+1}$ or $x_{n+1} \preceq x_{n}$ (i.e., $x_{n}$ and $x_{n+1}$ are comparable with respect to ' $\preceq$ ') for all $n \in \mathbb{N}$.

- The sequence $\left\{\mathbf{x}_{n}\right\}_{n \in \mathbb{N}}$ in $X^{m}$ is said to be a mixed $\preceq$-monotone sequence if and only if each sequence $\left\{x_{n}^{(k)}\right\}_{n \in \mathbb{N}}$ in $X$ is a mixed $\preceq$-monotone sequence for all $k=1, \ldots, m$.

- The sequence $\left\{\mathbf{x}_{n}\right\}_{n \in \mathbb{N}}$ in $X^{m}$ is said to be a mixed $\preccurlyeq_{I}$-monotone sequence if and only if $\mathbf{x}_{n} \preccurlyeq{ }_{I} \mathbf{x}_{n+1}$ or $\mathbf{x}_{n+1} \preccurlyeq_{I} \mathbf{x}_{n}$ (i.e., $\mathbf{x}_{n}$ and $\mathbf{x}_{n+1}$ are comparable with respect to ' $\preccurlyeq_{I}^{\prime}$ ') for all $n \in \mathbb{N}$.

Remark 2.4 Let $I$ and $J$ be a disjoint pair of $\{1,2, \ldots, m\}$. We have the following observations.

(a) $\left\{\mathbf{x}_{n}\right\}_{n \in \mathbb{N}}$ in $X^{m}$ is a mixed $\preccurlyeq_{I}$-monotone sequence if and only if it is a mixed $\preccurlyeq$-monotone sequence.

(b) If $\left\{\mathbf{x}_{n}\right\}_{n \in \mathbb{N}}$ in $X^{m}$ is a mixed $\preccurlyeq_{I}$-monotone sequence, then it is also a mixed $\preceq$-monotone sequence; that is, each sequence $\left\{x_{n}^{(k)}\right\}_{n \in \mathbb{N}}$ in $X$ is a mixed $\preceq$-monotone sequence for all $k=1, \ldots, m$.

(c) If $\left\{\mathbf{x}_{n}\right\}_{n \in \mathbb{N}}$ in $X^{m}$ is a mixed $\preceq$-monotone sequence, then given any $n \in \mathbb{N}$, there exists a disjoint pair of $I_{n}$ and $J_{n}$ (which depends on $n$ ) of $\{1, \ldots, m\}$ such that $\mathbf{x}_{n} \preccurlyeq I_{n} \mathbf{x}_{n+1}$ or $\mathbf{x}_{n+1} \preccurlyeq I_{n} \mathbf{x}_{n}$.

(d) $\left\{\mathbf{x}_{n}\right\}_{n \in \mathbb{N}}$ in $X^{m}$ is a mixed $\preceq$-monotone sequence if and only if, for each $n \in \mathbb{N}$, $\mathbf{x}_{n}$ and $\mathbf{x}_{n+1}$ are $\preceq$-mixed comparable

Definition 2.5 Let $I$ and $J$ be a disjoint pair of $\{1,2, \ldots, m\}$. Given a quasi-ordered set $(X, \preceq)$, we also consider the quasi-ordered set $\left(X^{m}, \preccurlyeq_{I}\right)$ defined in (1) and the function $\mathbf{f}:\left(X^{m}, \mathfrak{d}\right) \rightarrow\left(X^{m}, \mathfrak{d}\right)$.

- The function $\mathbf{f}$ is said to have the sequentially mixed $\preceq$-monotone property if and only if, given any mixed $\preceq$-monotone sequence $\left\{\mathbf{x}_{n}\right\}_{n \in \mathbb{N}}$ in $X^{m},\left\{\mathbf{f}\left(\mathbf{x}_{n}\right)\right\}_{n \in \mathbb{N}}$ is also a mixed $\preceq$-monotone sequence. 


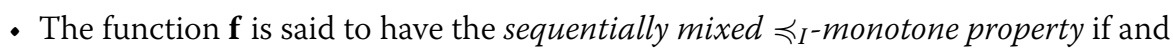
only if, given any mixed $\preccurlyeq_{I}$-monotone sequence $\left\{\mathbf{x}_{n}\right\}_{n \in \mathbb{N}}$ in $X^{m},\left\{\mathbf{f}\left(\mathbf{x}_{n}\right)\right\}_{n \in \mathbb{N}}$ is also a mixed $\preccurlyeq_{I}$-monotone sequence.

It is obvious that the identity function on $X^{m}$ has the sequentially mixed $\preccurlyeq_{I}$-monotone and $\preceq$-monotone property.

Let $X$ be a nonempty set. We consider the functions $\mathbf{F}: X^{m} \rightarrow X^{m}$ and $\mathbf{f}: X^{m} \rightarrow X^{m}$ satisfying $\mathbf{F}^{p}\left(X^{m}\right) \subseteq \mathbf{f}\left(X^{m}\right)$ for some $p \in \mathbb{N}$, where $\mathbf{F}^{p}(\mathbf{x})=\mathbf{F}\left(\mathbf{F}^{p-1}(\mathbf{x})\right)$ for any $\mathbf{x} \in X^{m}$. Therefore, we have $F_{k}^{p}(\mathbf{x})=F_{k}\left(\mathbf{F}^{p-1}(\mathbf{x})\right)$ for $k=1, \ldots, m$. Given an initial element $\mathbf{x}_{0}=$ $\left(x_{0}^{(1)}, x_{0}^{(2)}, \ldots, x_{0}^{(m)}\right) \in X^{m}$, where $x_{0}^{(k)} \in X$ for $k=1, \ldots, m$, since $\mathbf{F}^{p}\left(X^{m}\right) \subseteq \mathbf{f}\left(X^{m}\right)$, there exists $\mathbf{x}_{1} \in X^{m}$ such that $\mathbf{f}\left(\mathbf{x}_{1}\right)=\mathbf{F}^{p}\left(\mathbf{x}_{0}\right)$. Similarly, there also exists $\mathbf{x}_{2} \in X^{m}$ such that $\mathbf{f}\left(\mathbf{x}_{2}\right)=$ $\mathbf{F}^{p}\left(\mathbf{x}_{1}\right)$. Continuing this process, we can construct a sequence $\left\{\mathbf{x}_{n}\right\}_{n \in \mathbb{N}}$ such that

$$
\mathbf{f}\left(\mathbf{x}_{n}\right)=\mathbf{F}^{p}\left(\mathbf{x}_{n-1}\right)
$$

for all $n \in \mathbb{N}$; that is,

$$
f_{k}\left(\mathbf{x}_{n}\right)=f_{k}\left(x_{n}^{(1)}, \ldots, x_{n}^{(k)}, \ldots, x_{n}^{(m)}\right)=F_{k}^{p}\left(x_{n-1}^{(1)}, \ldots, x_{n-1}^{(k)}, \ldots, x_{n-1}^{(m)}\right)=F_{k}^{p}\left(\mathbf{x}_{n-1}\right)
$$

for all $k=1, \ldots, m$. We introduce the concepts of mixed monotone seed element as follows.

(A) We say that the initial element $\mathbf{x}_{0}$ is a mixed $\preceq$-monotone seed element of $X^{m}$ if and only if the sequence $\left\{\mathbf{x}_{n}\right\}_{n \in \mathbb{N}}$ constructed from (3) is a mixed $\preceq$-monotone sequence; that is, each sequence $\left\{x_{n}^{(k)}\right\}_{n \in \mathbb{N}}$ in $X$ is a mixed $\preceq$-monotone sequence for $k=1, \ldots, m$.

(B) Given a disjoint pair $I$ and $J$ of $\{1,2, \ldots, m\}$, we say that the initial element $\mathbf{x}_{0}$ is a mixed $\preccurlyeq_{I}$-monotone seed element of $X^{m}$ if and only if the sequence $\left\{\mathbf{x}_{n}\right\}_{n \in \mathbb{N}}$ constructed from (3) is a mixed $\preccurlyeq_{I}$-monotone sequence.

From observation (b) of Remark 2.4 , it follows that if $\mathbf{x}_{0}$ is a mixed $\preccurlyeq_{I}$-monotone seed element, then it is also a mixed $\preceq$-monotone seed element.

Example 2.6 Suppose that the initial element $\mathbf{x}_{0}$ can generate a sequence $\left\{\mathbf{x}_{n}\right\}_{n \in \mathbb{N}}$ such that, for each $k=1, \ldots, m$, the generated sequence $\left\{x_{n}^{(k)}\right\}_{n \in \mathbb{N}}$ is either $\preceq$-increasing or $\preceq$-decreasing. In this case, we define the disjoint pair $I$ and $J$ of $\{1,2, \ldots, m\}$ as follows:

$$
I=\left\{k \text { : the sequence }\left\{x_{n}^{(k)}\right\}_{n \in \mathbb{N}} \text { is } \preceq \text {-increasing }\right\} \quad \text { and } \quad J=\{1,2, \ldots, m\} \backslash I \text {. }
$$

It means that if $k \in J$, then the sequence $\left\{x_{n}^{(k)}\right\}_{n \in \mathbb{N}}$ is $\preceq$-decreasing. Therefore, the sequence $\left\{\mathbf{x}_{n}\right\}_{n \in \mathbb{N}}$ satisfies $\mathbf{x}_{n} \preccurlyeq_{I} \mathbf{x}_{n+1}$ for any $n \in \mathbb{N}$. In this case, the initial element $\mathbf{x}_{0}$ is a mixed $\preccurlyeq{ }_{I}$-monotone seed element with the disjoint pair $I$ and $J$ defined in (4).

Definition 2.7 Let $(X, d, \preceq)$ be a metric space endowed with a quasi-order ' $\preceq$ '. We say that $(X, d, \preceq)$ is mixed-monotonically complete if and only if each mixed $\preceq$-monotone Cauchy sequence $\left\{x_{n}\right\}_{n \in \mathbb{N}}$ in $X$ is convergent.

It is obvious that if the quasi-ordered metric space $(X, d, \preceq)$ is complete, then it is also mixed-monotonically complete. However, the converse is not necessarily true. 
For the metric space $(X, d)$, we can consider a product metric space $\left(X^{m}, \mathfrak{d}\right)$ in which the metric $\mathfrak{d}$ is induced by the original metric $d$. For example, the following distance functions

$$
\mathfrak{d}(\mathbf{x}, \mathbf{y})=\max _{k=1, \ldots, m}\left\{d\left(x^{(k)}, y^{(k)}\right)\right\}
$$

and

$$
\mathfrak{d}(\mathbf{x}, \mathbf{y})=\sum_{k=1}^{m} d\left(x^{(k)}, y^{(k)}\right)
$$

make $\left(X^{m}, \mathfrak{d}\right)$ to be the product metric spaces. For the general product metric $\mathfrak{d}$, we consider the following concepts.

- We say that the metrics $\mathfrak{d}$ and $d$ are compatible in the sense of preserving convergence if and only if, given a sequence $\left\{\mathbf{x}_{n}\right\}_{n \in \mathbb{N}}$ in $X^{m}$, the following statement holds:

$$
\mathfrak{d}\left(\mathbf{x}_{n}, \hat{\mathbf{x}}\right) \rightarrow 0 \quad \text { if and only if } \quad d\left(x_{n}^{(k)}, \hat{x}^{(k)}\right) \rightarrow 0 \quad \text { for all } k=1, \ldots, m
$$

- We say that the metrics $\mathfrak{d}$ and $d$ are compatible in the sense of preserving continuity if and only if, given any $\epsilon>0$, there exists a positive constant $\mathfrak{k}>0$ (which depends on $\epsilon$ ) such that the following statement holds:

$$
\mathfrak{d}(\mathbf{x}, \mathbf{y})<\epsilon \quad \text { if and only if } \quad d\left(x^{(k)}, y^{(k)}\right)<\mathfrak{k} \cdot \epsilon \quad \text { for all } k=1, \ldots, m
$$

We can check that the product metric $\mathfrak{d}$ defined in (5) or (6) is compatible with $d$ in the sense of preserving convergence and continuity.

Proposition 2.8 If $\mathfrak{d}$ and $d$ are compatible in the sense of preserving continuity, then $\mathfrak{d}$ and $d$ are compatible in the sense of preserving convergence.

Proof Suppose that $\mathfrak{d}\left(\mathbf{x}_{n}, \hat{\mathbf{x}}\right) \rightarrow 0$. By definition, given any $\epsilon>0$, there exists $n_{0} \in \mathbb{N}$ such that $\mathfrak{d}\left(\mathbf{x}_{n}, \hat{\mathbf{x}}\right)<\epsilon / \mathfrak{k}$ for all $n \geq n_{0}$, i.e., $d\left(x_{n}^{(k)}, \hat{x}^{(k)}\right)<\epsilon$ for all $k=1, \ldots, m$ and $n \geq n_{0}$. For the converse, given any $\epsilon>0$, there exist $n_{0}^{(k)} \in \mathbb{N}$ such that $d\left(x_{n}^{(k)}, \hat{x}^{(k)}\right)<\mathfrak{k} \cdot \epsilon$ for all $n \geq n_{0}^{(k)}$, where $k=1, \ldots, m$. Let

$$
n_{0}=\max _{k=1, \ldots, m} n_{0}^{(k)}
$$

It follows that $d\left(x_{n}^{(k)}, \hat{x}^{(k)}\right)<\mathfrak{k} \cdot \epsilon$ for all $n \geq n_{0}$ and all $k=1, \ldots, m$, i.e., $\mathfrak{d}\left(\mathbf{x}_{n}, \hat{\mathbf{x}}\right)<\epsilon$ for all $n \geq n_{0}$. This completes the proof.

Mizoguchi and Takahashi $[16,17]$ considered the mapping $\varphi:[0, \infty) \rightarrow[0,1)$ that satisfies the following condition:

$$
\limsup _{x \rightarrow c+} \varphi(x)<1 \quad \text { for all } c \in[0, \infty)
$$

in the contractive inequality, and generalized Nadler's fixed point theorem as shown in [18]. Suzuki [19] also gave a simple proof of the theorem obtained by Mizoguchi and Takahashi [16]. In this paper, we consider the following definition. 
Definition 2.9 We say that $\varphi:[0, \infty) \rightarrow[0,1)$ is a function of contractive factor if and only if, for any strictly decreasing sequence $\left\{x_{n}\right\}_{n \in \mathbb{N}}$ in $[0, \infty)$, we have

$$
0 \leq \sup _{n} \varphi\left(x_{n}\right)<1
$$

Using the routine arguments, we can show that the function $\varphi:[0, \infty) \rightarrow[0,1)$ satisfies (7) if and only if $\varphi$ is a function of contractive factor. Throughout this paper, we shall assume that the mapping $\varphi$ satisfies (8) in order to prove the various types of coincidence point theorems in the product space. The following lemma is obvious and useful for further discussion.

Lemma 2.10 Let $\varphi$ be a function of contractive factor. We define

$$
\kappa(t)=\frac{1+\varphi(t)}{2} .
$$

Then, for any strictly decreasing sequence $\left\{x_{n}\right\}_{n \in \mathbb{N}}$ in $[0, \infty)$, we have

$$
0 \leq \varphi(t)<\kappa(t)<1 \quad \text { for all } t \in[0, \infty) \text { and } 0<\sup _{n} \kappa\left(x_{n}\right)<1
$$

Let $(X, d)$ be a metric space, and let $\mathbf{F}:\left(X^{m}, \mathfrak{d}\right) \rightarrow\left(X^{m}, \mathfrak{d}\right)$ be a function defined on $\left(X^{m}, \mathfrak{d}\right)$ into itself. If $\mathbf{F}$ is continuous at $\hat{\mathbf{x}} \in X^{m}$, then, given $\epsilon>0$, there exists $\delta>0$ such that $\mathbf{x} \in X^{m}$ with $\mathfrak{d}(\hat{\mathbf{x}}, \mathbf{x})<\delta$ implies $\mathfrak{d}(\mathbf{F}(\hat{\mathbf{x}}), \mathbf{F}(\mathbf{x}))<\epsilon$.

Suppose that $\mathfrak{d}$ and $d$ are compatible in the sense of preserving continuity. Then $\mathbf{F}$ is continuous at $\hat{\mathbf{x}} \in X^{m}$ if and only if each $F_{k}$ is continuous at $\hat{\mathbf{x}}$ for $k=1, \ldots, m$. Indeed, it is obvious that if $\mathbf{F}$ is continuous at $\hat{\mathbf{x}} \in X^{m}$, then each $F_{k}$ is continuous at $\hat{\mathbf{x}}$ for $k=$ $1, \ldots, m$. For the converse, given any $\epsilon>0$, there exists $\delta_{k}>0$ such that $\mathfrak{d}(\hat{\mathbf{x}}, \mathbf{x})<\delta_{k}$ implies $d\left(F_{k}(\hat{\mathbf{x}}), F_{k}(\mathbf{x})\right)<\mathfrak{k} \cdot \epsilon$, where $k=1, \ldots, m$. Let

$$
\delta=\min _{k=1, \ldots, m} \delta_{k}
$$

It follows that $\mathfrak{d}(\hat{\mathbf{x}}, \mathbf{x})<\delta$ implies $d\left(F_{k}(\hat{\mathbf{x}}), F_{k}(\mathbf{x})\right)<\mathfrak{k} \cdot \epsilon$ for all $k=1, \ldots, m$, i.e., $\mathfrak{d}(\mathbf{F}(\hat{\mathbf{x}})$, $\mathbf{F}(\mathbf{x}))<\epsilon$. Next, we propose another concept of continuity.

Definition 2.11 Let $(X, d)$ be a metric space, and let $\left(X^{m}, \mathfrak{d}\right)$ be the corresponding product metric space. Let $\mathbf{F}:\left(X^{m}, \mathfrak{d}\right) \rightarrow\left(X^{m}, \mathfrak{d}\right)$ and $\mathbf{f}:\left(X^{m}, \mathfrak{d}\right) \rightarrow\left(X^{m}, \mathfrak{d}\right)$ be functions defined on $\left(X^{m}, \mathfrak{d}\right)$ into itself. We say that $\mathbf{F}$ is continuous with respect to $\mathbf{f}$ at $\hat{\mathbf{x}} \in X^{m}$ if and only if, given any $\epsilon>0$, there exists $\delta>0$ such that $\mathbf{x} \in X^{m}$ with $\mathfrak{d}(\hat{\mathbf{x}}, \mathbf{f}(\mathbf{x}))<\delta$ implies $\mathfrak{d}(\mathbf{F}(\hat{\mathbf{x}}), \mathbf{F}(\mathbf{x}))<\epsilon$. We say that $\mathbf{F}$ is continuous with respect to $\mathbf{f}$ on $X^{m}$ if and only if it is continuous with respect to $\mathbf{f}$ at each $\hat{\mathbf{x}} \in X^{m}$.

It is obvious that if the function $\mathbf{F}$ is continuous at $\hat{\mathbf{x}}$ with respect to the identity function, then it is also continuous at $\hat{\mathbf{x}}$.

Proposition 2.12 Let $(X, d)$ be a metric space, and let $\mathbf{F}:\left(X^{m}, \mathfrak{d}\right) \rightarrow\left(X^{m}, \mathfrak{d}\right)$ and $\mathbf{f}$ : $\left(X^{m}, \mathfrak{d}\right) \rightarrow\left(X^{m}, \mathfrak{d}\right)$ be functions defined on $\left(X^{m}, \mathfrak{d}\right)$ into itself. Suppose that $\mathfrak{d}$ and $d$ are compatible in the sense of preserving continuity. Then $\mathbf{F}$ is continuous with respect to $\mathbf{f}$ at $\hat{\mathbf{x}} \in X^{m}$ 
if and only if, given any $\epsilon>0$, there exists $\delta>0$ such that $\mathbf{x} \in X^{m}$ with $d\left(\hat{x}^{(k)}, f_{k}(\mathbf{x})\right)<\delta$ for all $k=1, \ldots, m$ implies $d\left(F_{k}(\hat{\mathbf{x}}), F_{k}(\mathbf{x})\right)<\epsilon$ for all $k=1, \ldots, m$.

Proof Suppose that $\mathbf{F}$ is continuous with respect to $\mathbf{f}$ at $\hat{\mathbf{x}}$. By definition, given any $\epsilon>0$, there exists $\hat{\delta}>0$ such that $\mathbf{x} \in X^{m}$ with $\mathfrak{d}(\hat{\mathbf{x}}, \mathbf{f}(\mathbf{x}))<\hat{\delta}$ implies $\mathfrak{d}(\mathbf{F}(\hat{\mathbf{x}}), \mathbf{F}(\mathbf{x}))<\epsilon / \mathfrak{k}$. Let $\delta=\mathfrak{k} \cdot \hat{\delta}$. It follows that $d\left(\hat{x}^{(k)}, f_{k}(\mathbf{x})\right)<\delta$ for all $k=1, \ldots, m$ if and only if $\mathfrak{d}(\hat{\mathbf{x}}, \mathbf{f}(\mathbf{x}))<\hat{\delta}$, which implies $\mathfrak{d}(\mathbf{F}(\hat{\mathbf{x}}), \mathbf{F}(\mathbf{x}))<\epsilon / \mathfrak{k}$, i.e., $d\left(F_{k}(\hat{\mathbf{x}}), F_{k}(\mathbf{x})\right)<\epsilon$ for all $k=1, \ldots, m$. For the converse, given any $\epsilon>$ 0 , there exists $\delta^{*}>0$ such that $d\left(\hat{x}^{(k)}, f_{k}(\mathbf{x})\right)<\delta^{*}$ for all $k=1, \ldots, m$ implies $d\left(F_{k}(\hat{\mathbf{x}}), F_{k}(\mathbf{x})\right)<$ $\mathfrak{k} \cdot \epsilon$ for all $k=1, \ldots, m$. Let $\delta=\delta^{*} / \mathfrak{k}$. It follows that $\mathfrak{d}(\hat{\mathbf{x}}, \mathbf{f}(\mathbf{x}))<\delta$ if and only if $d\left(\hat{x}^{(k)}, f_{k}(\mathbf{x})\right)<$ $\delta^{*}$ for all $k=1, \ldots, m$, which implies $d\left(F_{k}(\hat{\mathbf{x}}), F_{k}(\mathbf{x})\right)<\mathfrak{k} \cdot \epsilon$ for all $k=1, \ldots, m$, i.e., $\mathfrak{d}(\mathbf{F}(\hat{\mathbf{x}})$, $\mathbf{F}(\mathbf{x}))<\epsilon$. This completes the proof.

Lemma 2.13 Let $(X, d)$ be a metric space. If $x_{n} \rightarrow x$ as $n \rightarrow \infty$ with respect to the metric $d$, then, given any fixed $y \in X, d\left(x_{n}, y\right) \rightarrow d(x, y)$ as $n \rightarrow \infty$.

Theorem 2.14 Suppose that the quasi-ordered metric space $(X, d, \preceq)$ is mixed-monotonically complete, and that the metrics $\mathfrak{d}$ and $d$ are compatible in the sense of preserving continuity. Consider the functions $\mathbf{F}:\left(X^{m}, \mathfrak{d}\right) \rightarrow\left(X^{m}, \mathfrak{d}\right)$ and $\mathbf{f}:\left(X^{m}, \mathfrak{d}\right) \rightarrow\left(X^{m}, \mathfrak{d}\right)$ satisfying $\mathbf{F}^{p}\left(X^{m}\right) \subseteq \mathbf{f}\left(X^{m}\right)$ for some $p \in \mathbb{N}$. Let $\mathbf{x}_{0}$ be a mixed $\preceq$-monotone seed element in $X^{m}$. Assume that the functions $\mathbf{F}$ and $\mathbf{f}$ satisfy the following conditions:

- $\mathbf{F}$ and $\mathbf{f}$ are commutative;

- $\mathbf{f}$ has the sequentially mixed $\preceq$-monotone property;

- $\mathbf{F}^{p}$ is continuous with respect to $\mathbf{f}$ on $X^{m}$;

- each $f_{k}$ is continuous on $X^{m}$ for $k=1, \ldots, m$.

Suppose that there exist a function $\rho: X^{m} \times X^{m} \rightarrow \mathbb{R}_{+}$and a function of contractive factor $\varphi:[0, \infty) \rightarrow[0,1)$ such that, for any two $\preceq$-mixed comparable elements $\mathbf{x}$ and $\mathbf{y}$ in $X^{m}$, the following inequalities are satisfied:

$$
\rho(\mathbf{x}, \mathbf{y}) \leq \sum_{k=1}^{m} d\left(x^{(k)}, y^{(k)}\right)
$$

and, for each $k=1, \ldots, m$,

$$
d\left(F_{k}^{p}(\mathbf{x}), F_{k}^{p}(\mathbf{y})\right) \leq \frac{1}{m} \cdot \varphi(\rho(\mathbf{f}(\mathbf{x}), \mathbf{f}(\mathbf{y}))) \cdot \rho(\mathbf{f}(\mathbf{x}), \mathbf{f}(\mathbf{y})) .
$$

Then $\mathbf{F}^{p}$ has a fixed point $\hat{\mathbf{x}}$ such that each component $\hat{x}^{(k)}$ of $\hat{\mathbf{x}}$ is the limit of the sequence $\left\{f_{k}\left(\mathbf{x}_{n}\right)\right\}_{n \in \mathbb{N}}$ constructed in (3) for all $k=1, \ldots, m$.

Proof We consider the sequence $\left\{\mathbf{x}_{n}\right\}_{n \in \mathbb{N}}$ constructed from (3). Since $\mathbf{x}_{0}$ is a mixed $\preceq$-monotone seed element in $X^{m}$, i.e., $\left\{\mathbf{x}_{n}\right\}_{n \in \mathbb{N}}$ is a mixed $\preceq$-monotone sequence, from observation (d) of Remark 2.4, it follows that, for each $n \in \mathbb{N}, \mathbf{x}_{n}$ and $\mathbf{x}_{n+1}$ are $\preceq$-mixed comparable. According to inequalities (10), we obtain

$$
\begin{aligned}
d\left(f_{k}\left(\mathbf{x}_{n+1}\right), f_{k}\left(\mathbf{x}_{n}\right)\right) & =d\left(F_{k}^{p}\left(\mathbf{x}_{n}\right), F_{k}^{P}\left(\mathbf{x}_{n-1}\right)\right) \\
& \leq \frac{1}{m} \varphi\left(\rho\left(\mathbf{f}\left(\mathbf{x}_{n}\right), \mathbf{f}\left(\mathbf{x}_{n-1}\right)\right)\right) \cdot \rho\left(\mathbf{f}\left(\mathbf{x}_{n}\right), \mathbf{f}\left(\mathbf{x}_{n-1}\right)\right) .
\end{aligned}
$$


Since $\mathbf{f}$ has the sequentially mixed $\preceq$-monotone property, we see that $\left\{\mathbf{f}\left(\mathbf{x}_{n}\right)\right\}_{n \in \mathbb{N}}$ is a mixed $\preceq$-monotone sequence. From observation (d) of Remark 2.4, it follows that, for each $n \in \mathbb{N}$, $\mathbf{f}\left(\mathbf{x}_{n}\right)$ and $\mathbf{f}\left(\mathbf{x}_{n+1}\right)$ are $\preceq$-mixed comparable. Then we have

$$
\begin{aligned}
\rho\left(\mathbf{f}\left(\mathbf{x}_{n+1}\right), \mathbf{f}\left(\mathbf{x}_{n}\right)\right) & \leq \sum_{k=1}^{m} d\left(f_{k}\left(\mathbf{x}_{n+1}\right), f_{k}\left(\mathbf{x}_{n}\right)\right) \quad(\text { by }(9)) \\
& \leq \varphi\left(\rho\left(\mathbf{f}\left(\mathbf{x}_{n}\right), \mathbf{f}\left(\mathbf{x}_{n-1}\right)\right)\right) \cdot \rho\left(\mathbf{f}\left(\mathbf{x}_{n}\right), \mathbf{f}\left(\mathbf{x}_{n-1}\right)\right) \quad(\text { by }(11)) .
\end{aligned}
$$

Let

$$
\xi_{n}=\rho\left(\mathbf{f}\left(\mathbf{x}_{n}\right), \mathbf{f}\left(\mathbf{x}_{n-1}\right)\right) \quad \text { and } \quad \kappa(t)=\frac{1+\varphi(t)}{2}
$$

Using (11) and Lemma 2.10, we obtain

$$
d\left(f_{k}\left(\mathbf{x}_{n+1}\right), f_{k}\left(\mathbf{x}_{n}\right)\right) \leq \frac{1}{m} \varphi\left(\xi_{n}\right) \cdot \xi_{n}<\frac{1}{m} \kappa\left(\xi_{n}\right) \cdot \xi_{n}
$$

Using (12) and Lemma 2.10, we also obtain

$$
\xi_{n+1} \leq \varphi\left(\xi_{n}\right) \cdot \xi_{n}<\kappa\left(\xi_{n}\right) \cdot \xi_{n}
$$

Since $0<\gamma=\sup _{n} \kappa\left(\xi_{n}\right)<1$ by Lemma 2.10 again, from (13) and (14), it follows that

$$
d\left(f_{k}\left(\mathbf{x}_{n+1}\right), f_{k}\left(\mathbf{x}_{n}\right)\right)<\frac{\gamma}{m} \cdot \xi_{n} \quad \text { and } \quad \xi_{n+1}<\gamma \cdot \xi_{n}
$$

which implies

$$
d\left(f_{k}\left(\mathbf{x}_{n+1}\right), f_{k}\left(\mathbf{x}_{n}\right)\right)<\frac{\gamma^{n}}{m} \xi_{1}
$$

For $n_{1}, n_{2} \in \mathbb{N}$ with $n_{1}>n_{2}$, since $0<\gamma<1$, from (15) we have

$$
\begin{aligned}
d\left(f_{k}\left(\mathbf{x}_{n_{1}}\right), f_{k}\left(\mathbf{x}_{n_{2}}\right)\right) & \leq \sum_{j=n_{2}}^{n_{1}-1} d\left(f_{k}\left(\mathbf{x}_{j+1}\right), f_{k}\left(\mathbf{x}_{j}\right)\right) \\
& <\frac{\xi_{1}}{m} \cdot \frac{\gamma^{n_{2}}\left(1-\gamma^{n_{1}-n_{2}}\right)}{1-\gamma} \\
& <\frac{\xi_{1}}{m} \cdot \frac{\gamma^{n_{2}}}{1-\gamma} \rightarrow 0 \quad \text { as } n_{2} \rightarrow \infty
\end{aligned}
$$

which also says that $\left\{f_{k}\left(\mathbf{x}_{n}\right)\right\}$ is a Cauchy sequence in $X$ for any fixed $k$. Since $\mathbf{f}$ has the sequentially mixed $\preceq$-monotone property, i.e., $\left\{f_{k}\left(\mathbf{x}_{n}\right)\right\}_{n \in \mathbb{N}}$ is a mixed $\preceq$-monotone Cauchy sequence for $k=1, \ldots, m$, by the mixed $\preceq$-monotone completeness of $X$, there exists $\hat{x}^{(k)} \in X$ such that $f_{k}\left(\mathbf{x}_{n}\right) \rightarrow \hat{x}^{(k)}$ as $n \rightarrow \infty$ for $k=1, \ldots, m$. Since the metrics $\mathfrak{d}$ and $d$ are compatible in the sense of preserving continuity, by Proposition 2.8, it follows that $\mathbf{f}\left(\mathbf{x}_{n}\right) \rightarrow \hat{\mathbf{x}}$ as $n \rightarrow \infty$. Since each $f_{k}$ is continuous on $X^{m}$, we also have

$$
f_{k}\left(\mathbf{f}\left(\mathbf{x}_{n}\right)\right) \rightarrow f_{k}(\hat{\mathbf{x}}) \quad \text { as } n \rightarrow \infty
$$


Since $\mathbf{F}^{p}$ is continuous with respect to $\mathbf{f}$ on $X^{m}$, by Proposition 2.12, given any $\epsilon>0$, there exists $\delta>0$ such that $\mathbf{x} \in X^{m}$ with $d\left(\hat{x}^{(k)}, f_{k}(\mathbf{x})\right)<\delta$ for all $k=1, \ldots, m$ implies

$$
d\left(F_{k}^{p}(\hat{\mathbf{x}}), F_{k}^{p}(\mathbf{x})\right)<\frac{\epsilon}{2} \quad \text { for all } k=1, \ldots, m .
$$

Since $f_{k}\left(\mathbf{x}_{n}\right) \rightarrow \hat{x}^{(k)}$ as $n \rightarrow \infty$ for all $k=1, \ldots, m$, given $\zeta=\min \{\epsilon / 2, \delta\}>0$, there exists $n_{0} \in \mathbb{N}$ such that

$$
d\left(f_{k}\left(\mathbf{x}_{n}\right), \hat{x}^{(k)}\right)<\zeta \leq \delta \quad \text { for all } n \in \mathbb{N} \text { with } n \geq n_{0} \text { and for all } k=1, \ldots, m \text {. }
$$

For each $n \geq n_{0}$, by (16) and (17), it follows that

$$
d\left(F_{k}^{p}(\hat{\mathbf{x}}), F_{k}^{p}\left(\mathbf{x}_{n}\right)\right)<\frac{\epsilon}{2} \quad \text { for all } k=1, \ldots, m
$$

Therefore, we obtain

$$
\begin{aligned}
d\left(F_{k}^{p}(\hat{\mathbf{x}}), \hat{x}^{(k)}\right) & \leq d\left(F_{k}^{p}(\hat{\mathbf{x}}), f_{k}\left(\mathbf{x}_{n_{0}+1}\right)\right)+d\left(f_{k}\left(\mathbf{x}_{n_{0}+1}\right), \hat{x}^{(k)}\right) \\
& =d\left(F_{k}^{p}(\hat{\mathbf{x}}), F_{k}^{p}\left(\mathbf{x}_{n_{0}}\right)\right)+d\left(f_{k}\left(\mathbf{x}_{n_{0}+1}\right), \hat{x}^{(k)}\right) \\
& <\frac{\epsilon}{2}+\zeta \quad(\text { by }(17) \text { and }(18)) \\
& \leq \epsilon \quad \text { for all } k=1, \ldots, m .
\end{aligned}
$$

Since $\epsilon$ is any positive number, we conclude that $d\left(F_{k}^{p}(\hat{\mathbf{x}}), \hat{x}^{(k)}\right)=0$ for all $k=1, \ldots, m$, which also says that $F_{k}^{p}(\hat{\mathbf{x}})=\hat{x}^{(k)}$ for all $k=1, \ldots, m$, i.e., $\mathbf{F}^{p}(\hat{\mathbf{x}})=\hat{\mathbf{x}}$. This completes the proof.

Remark 2.15 We have the following observations.

- In Theorem 2.14, if we assume that the quasi-ordered metric space $(X, d, \preceq)$ is complete (not mixed-monotonically complete), then the assumption for $\mathbf{f}$ having the sequentially mixed $\preceq$-monotone property can be dropped, since the proof is still valid in this case.

- The assumption for inequalities (9) and (10) is weak since we just assume that it is satisfied for $\preceq$-mixed comparable elements. In other words, if $\mathbf{x}$ and $\mathbf{y}$ are not $\preceq$-mixed comparable, we do not need to check inequalities (9) and (10).

By considering the mixed $\preccurlyeq_{I}$-monotone seed element instead of mixed $\preceq$-monotone seed element, the assumptions for inequalities (9) and (10) can be weakened, which is shown below.

Theorem 2.16 Suppose that the quasi-ordered metric space $(X, d, \preceq)$ is mixed-monotonically complete, and that the metrics $\mathfrak{d}$ and $d$ are compatible in the sense of preserving continuity. Let $I$ and $J$ be any disjoint pair of $\{1,2, \ldots, m\}$. Consider the functions $\mathbf{F}:\left(X^{m}, \mathfrak{d}, \preccurlyeq_{I}\right) \rightarrow\left(X^{m}, \mathfrak{d}, \preccurlyeq_{I}\right)$ and $\mathbf{f}:\left(X^{m}, \mathfrak{d}, \preccurlyeq_{I}\right) \rightarrow\left(X^{m}, \mathfrak{d}, \preccurlyeq_{I}\right)$ satisfying $\mathbf{F}^{p}\left(X^{m}\right) \subseteq \mathbf{f}\left(X^{m}\right)$ for some $p \in \mathbb{N}$. Let $\mathbf{x}_{0}$ be a mixed $\preccurlyeq{ }_{I}$-monotone seed element in $X^{m}$. Assume that the functions $\mathbf{F}$ and $\mathbf{f}$ satisfy the following conditions:

- $\mathbf{F}$ and $\mathbf{f}$ are commutative; 
- f has the sequentially mixed $\preccurlyeq I_{\text {-monotone property or the sequentially mixed }}$ $\preceq$-monotone property;

- $\mathbf{F}^{p}$ is continuous with respect to $\mathbf{f}$ on $X^{m}$;

- each $f_{k}$ is continuous on $X^{m}$ for $k=1, \ldots, m$.

Suppose that there exist a function $\rho: X^{m} \times X^{m} \rightarrow \mathbb{R}_{+}$and a function of contractive factor $\varphi:[0, \infty) \rightarrow[0,1)$ such that, for any $\mathbf{x}, \mathbf{y} \in X^{m}$ with $\mathbf{y} \preccurlyeq_{I} \mathbf{x}$ or $\mathbf{x} \preccurlyeq_{I} \mathbf{y}$, the following inequalities are satisfied:

$$
\rho(\mathbf{x}, \mathbf{y}) \leq \sum_{k=1}^{m} d\left(x^{(k)}, y^{(k)}\right)
$$

and, for each $k=1, \ldots, m$,

$$
d\left(F_{k}^{p}(\mathbf{x}), F_{k}^{p}(\mathbf{y})\right) \leq \frac{1}{m} \cdot \varphi(\rho(\mathbf{f}(\mathbf{x}), \mathbf{f}(\mathbf{y}))) \cdot \rho(\mathbf{f}(\mathbf{x}), \mathbf{f}(\mathbf{y})) .
$$

Then $\mathbf{F}^{p}$ has a fixed point $\hat{\mathbf{x}}$ such that each component $\hat{x}^{(k)}$ of $\hat{\mathbf{x}}$ is the limit of the sequence $\left\{f_{k}\left(\mathbf{x}_{n}\right)\right\}_{n \in \mathbb{N}}$ constructed in (3) for all $k=1, \ldots, m$.

Proof We consider the sequence $\left\{\mathbf{x}_{n}\right\}_{n \in \mathbb{N}}$ constructed from (3). Since $\mathbf{x}_{0}$ is a mixed $\preccurlyeq_{I}$-monotone seed element in $X^{m}$, it follows that $\left\{\mathbf{x}_{n}\right\}_{n \in \mathbb{N}}$ is a mixed $\preccurlyeq_{I}$-monotone sequence, i.e., for each $n \in \mathbb{N}, \mathbf{x}_{n-1} \preccurlyeq_{I} \mathbf{x}_{n}$ or $\mathbf{x}_{n} \preccurlyeq_{I} \mathbf{x}_{n-1}$. According to inequalities (20), we obtain

$$
\begin{aligned}
d\left(f_{k}\left(\mathbf{x}_{n+1}\right), f_{k}\left(\mathbf{x}_{n}\right)\right) & =d\left(F_{k}^{p}\left(\mathbf{x}_{n}\right), F_{k}^{P}\left(\mathbf{x}_{n-1}\right)\right) \\
& \leq \frac{1}{m} \varphi\left(\rho\left(\mathbf{f}\left(\mathbf{x}_{n}\right), \mathbf{f}\left(\mathbf{x}_{n-1}\right)\right)\right) \cdot \rho\left(\mathbf{f}\left(\mathbf{x}_{n}\right), \mathbf{f}\left(\mathbf{x}_{n-1}\right)\right) .
\end{aligned}
$$

Using the argument in the proof of Theorem 2.14, we can show that $\left\{f_{k}\left(\mathbf{x}_{n}\right)\right\}_{n \in \mathbb{N}}$ is a Cauchy sequence in $X$ for any fixed $k$. Now, we consider the following cases.

- Suppose that $\mathbf{f}$ has the sequentially mixed $\preccurlyeq_{I}$-monotone property. We see that $\left\{\mathbf{f}\left(\mathbf{x}_{n}\right)\right\}_{n \in \mathbb{N}}$ is a mixed $\preccurlyeq_{I}$-monotone sequence; that is, for each $n \in \mathbb{N}, \mathbf{f}\left(\mathbf{x}_{n}\right) \preccurlyeq_{I} \mathbf{f}\left(\mathbf{x}_{n+1}\right)$ or $\mathbf{f}\left(\mathbf{x}_{n+1}\right) \preccurlyeq{ }_{I} \mathbf{f}\left(\mathbf{x}_{n}\right)$. Since $\left\{f_{k}\left(\mathbf{x}_{n}\right)\right\}_{n \in \mathbb{N}}$ is a Cauchy sequence in $X$ for any fixed $k$, from observation (b) of Remark 2.4, we also see that $\left\{f_{k}\left(\mathbf{x}_{n}\right)\right\}_{n \in \mathbb{N}}$ is a mixed $\preceq$-monotone Cauchy sequence for $k=1, \ldots, m$.

- Suppose that $\mathbf{f}$ has the sequentially mixed $\preceq$-monotone property. Since $\left\{\mathbf{x}_{n}\right\}_{n \in \mathbb{N}}$ is a mixed $\preccurlyeq_{I}$-monotone sequence, by part (b) of Remark 2.4, it follows that $\left\{x_{n}^{(k)}\right\}_{n \in \mathbb{N}}$ in $X$ is a mixed $\preceq$-monotone sequence for all $k=1, \ldots, m$. Therefore, we obtain that $\left\{f_{k}\left(\mathbf{x}_{n}\right)\right\}_{n \in \mathbb{N}}$ is a mixed $\preceq$-monotone Cauchy sequence for $k=1, \ldots, m$.

By the mixed $\preceq$-monotone completeness of $X$, there exists $\hat{x}^{(k)} \in X$ such that $f_{k}\left(\mathbf{x}_{n}\right) \rightarrow \hat{x}^{(k)}$ as $n \rightarrow \infty$ for $k=1, \ldots, m$. The remaining proof follows from the same argument in the proof of Theorem 2.14. This completes the proof.

Remark 2.17 We have the following observations.

- In Theorem 2.16, if we assume that the quasi-ordered metric space $(X, d, \preceq)$ is complete (not mixed-monotonically complete), then the assumption for $\mathbf{f}$ having the sequentially mixed $\preccurlyeq{ }_{I}$-monotone or $\preceq$-monotone property can be dropped, since the proof is still valid in this case. 
- From observation (a) of Remark 2.2, we see that the assumption for inequalities (20) and (19) are indeed weakened by comparing to inequalities (9) and (10).

Next, we shall study the coincidence point without considering the continuity of $\mathbf{F}^{p}$. However, we need to introduce the concept of mixed-monotone convergence given below.

Definition 2.18 Let $(X, d, \preceq)$ be a metric space endowed with a quasi-order ' $\preceq$ '. We say that $(X, d, \preceq)$ preserves the mixed-monotone convergence if and only if, for each mixed $\preceq$-monotone sequence $\left\{x_{n}\right\}_{n \in \mathbb{N}}$ that converges to $\hat{x}$, we have $x_{n} \preceq \hat{x}$ or $\hat{x} \preceq x_{n}$ for each $n \in \mathbb{N}$.

Remark 2.19 Let $(X, d, \preceq)$ be a metric space endowed with a quasi-order ' $\preceq$ ' and preserve the mixed-monotone convergence. Suppose that $\left\{\mathbf{x}_{n}\right\}_{n \in \mathbb{N}}$ is a sequence in the product space $X^{m}$ such that each sequence $\left\{x_{n}^{(k)}\right\}_{n \in \mathbb{N}}$ is a mixed $\preceq$-monotone convergence sequence with limit point $\hat{x}^{(k)}$ for $k=1, \ldots, m$. Then we have the following observations.

(a) For each $n \in \mathbb{N}, \mathbf{x}_{n}$ and $\hat{\mathbf{x}}$ are $\preceq$-mixed comparable.

(b) For each $n \in \mathbb{N}$, there exists a disjoint pair $I_{n}$ and $J_{n}$ (that depend on $n$ ) of $\{1, \ldots, m\}$ such that $\mathbf{x}_{n} \preccurlyeq I_{n} \hat{\mathbf{x}}$ or $\hat{\mathbf{x}} \preccurlyeq I_{n} \mathbf{x}_{n}$, where $I_{n}$ or $J_{n}$ is allowed to be an empty set.

Definition 2.20 Let $I$ and $J$ be a disjoint pair of $\{1,2, \ldots, m\}$. Given a quasi-ordered set $(X, \preceq)$, we also consider the quasi-ordered set $\left(X^{m}, \preccurlyeq_{I}\right)$ defined in (1), and the function $\mathbf{f}:\left(X^{m}, \preccurlyeq I\right) \rightarrow\left(X^{m}, \preccurlyeq{ }_{I}\right)$.

- We say that the function $\mathbf{f}$ has the $\preceq$-comparable property if and only if, given any two $\preceq$-comparable elements $\mathbf{x}$ and $\mathbf{y}$ in $X^{m}$, the function values $\mathbf{f}(\mathbf{x})$ and $\mathbf{f}(\mathbf{y})$ are $\preceq$-comparable.

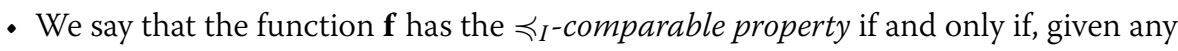
two $\preccurlyeq_{I}$-comparable elements $\mathbf{x}$ and $\mathbf{y}$ in $X^{m}$, the function values $\mathbf{f}(\mathbf{x})$ and $\mathbf{f}(\mathbf{y})$ are $\preccurlyeq I^{\text {-comparable. }}$

Since we shall study the coincidence point without considering the continuity of $\mathbf{F}^{p}$, we can also consider the assumption that the metrics $\mathfrak{d}$ and $d$ are compatible in the sense of preserving convergence, which is weaker than that of preserving continuity considered in the previous theorems.

Theorem 2.21 Suppose that the quasi-ordered metric space $(X, d, \preceq)$ is mixed-monotonically complete and preserves the mixed-monotone convergence. Assume that the metrics $\mathfrak{d}$ and $d$ are compatible in the sense of preserving convergence. Consider the functions $\mathbf{F}$ : $\left(X^{m}, \mathfrak{d}\right) \rightarrow\left(X^{m}, \mathfrak{d}\right)$ and $\mathbf{f}:\left(X^{m}, \mathfrak{d}\right) \rightarrow\left(X^{m}, \mathfrak{d}\right)$ satisfying $\mathbf{F}^{p}\left(X^{m}\right) \subseteq \mathbf{f}\left(X^{m}\right)$ for some $p \in \mathbb{N}$. Let $\mathbf{x}_{0}$ be a mixed $\preceq$-monotone seed element in $X^{m}$. Assume that the functions $\mathbf{F}$ and $\mathbf{f}$ satisfy the following conditions:

- $\mathbf{F}$ and $\mathbf{f}$ are commutative;

- $\mathbf{f}$ has the $\preceq$-comparable property and the sequentially mixed $\preceq$-monotone property;

- each $f_{k}$ is continuous on $X^{m}$ for $k=1, \ldots, m$.

Suppose that there exist a function $\rho: X^{m} \times X^{m} \rightarrow \mathbb{R}_{+}$and a function of contractive factor $\varphi:[0, \infty) \rightarrow[0,1)$ such that, for any two $\preceq$-mixed comparable elements $\mathbf{x}$ and $\mathbf{y}$ in $X^{m}$, the following inequalities are satisfied:

$$
\rho(\mathbf{x}, \mathbf{y}) \leq \sum_{k=1}^{m} d\left(x^{(k)}, y^{(k)}\right)
$$


and, for each $k=1, \ldots, m$,

$$
d\left(F_{k}^{p}(\mathbf{x}), F_{k}^{p}(\mathbf{y})\right) \leq \frac{1}{m} \cdot \varphi(\rho(\mathbf{f}(\mathbf{x}), \mathbf{f}(\mathbf{y}))) \cdot \rho(\mathbf{f}(\mathbf{x}), \mathbf{f}(\mathbf{y})) .
$$

Then the following statements hold true.

(i) There exists $\hat{\mathbf{x}} \in X^{m}$ of $\mathbf{F}$ such that $\mathbf{F}^{p}(\hat{\mathbf{x}})=\mathbf{f}(\hat{\mathbf{x}})$. If $p=1$, then $\hat{\mathbf{x}}$ is a coincidence point of $\mathbf{F}$ and $\mathbf{f}$.

(ii) If there exists $\hat{\mathbf{y}} \in X^{m}$ such that $\hat{\mathbf{x}}$ and $\hat{\mathbf{y}}$ are $\preceq$-mixed comparable satisfying $\mathbf{F}^{p}(\hat{\mathbf{y}})=\mathbf{f}(\hat{\mathbf{y}})$, then $\mathbf{f}(\hat{\mathbf{x}})=\mathbf{f}(\hat{\mathbf{y}})$.

(iii) Suppose that $\hat{\mathbf{x}}$ is obtained from part (i). If $\hat{\mathbf{x}}$ and $\mathbf{F}(\hat{\mathbf{x}})$ are $\preceq$-mixed comparable, then $\mathbf{f}^{q}(\hat{\mathbf{x}})$ is a fixed point of $\mathbf{F}$ for any $q \in \mathbb{N}$.

Moreover, each component $\hat{x}^{(k)}$ of $\hat{\mathbf{x}}$ is the limit of the sequence $\left\{f_{k}\left(\mathbf{x}_{n}\right)\right\}_{n \in \mathbb{N}}$ constructed in (3) for all $k=1, \ldots, m$.

Proof From the proof of Theorem 2.14, we can construct a sequence $\left\{\mathbf{x}_{n}\right\}_{n \in \mathbb{N}}$ in $X^{m}$ such that $f_{k}\left(\mathbf{x}_{n}\right) \rightarrow \hat{x}^{(k)}$ and $f_{k}\left(\mathbf{f}\left(\mathbf{x}_{n}\right)\right) \rightarrow f_{k}(\hat{\mathbf{x}})$ as $n \rightarrow \infty$, where $\left\{f_{k}\left(\mathbf{x}_{n}\right)\right\}_{n \in \mathbb{N}}$ is a mixed $\preceq$-monotone sequence for all $k=1, \ldots, m$. Since $f_{k}\left(\mathbf{f}\left(\mathbf{x}_{n}\right)\right) \rightarrow f_{k}(\hat{\mathbf{x}})$ as $n \rightarrow \infty$, given any $\epsilon>0$, there exists $n_{0} \in \mathbb{N}$ such that

$$
d\left(f_{k}\left(\mathbf{f}\left(\mathbf{x}_{n}\right)\right), f_{k}(\hat{\mathbf{x}})\right)<\frac{\epsilon}{2}
$$

for all $n \in \mathbb{N}$ with $n \geq n_{0}$ and for all $k=1, \ldots, m$. Since $\left\{f_{k}\left(\mathbf{x}_{n}\right)\right\}_{n \in \mathbb{N}}$ is a mixed $\preceq$-monotone convergent sequence for all $k=1, \ldots, m$, from observation (a) of Remark 2.19, we see that, for each $n \in \mathbb{N}, \mathbf{f}\left(\mathbf{x}_{n}\right)$ and $\hat{\mathbf{x}}$ are $\preceq$-mixed comparable. Since $\mathbf{f}$ has the $\preceq$-comparable property, it follows that $\mathbf{f}\left(\mathbf{f}\left(\mathbf{x}_{n}\right)\right)$ and $\mathbf{f}(\hat{\mathbf{x}})$ are $\preceq$-mixed comparable. For each $n \geq n_{0}$, it follows that

$$
\begin{aligned}
d\left(F_{k}^{p}(\hat{\mathbf{x}}), F_{k}^{p}\left(\mathbf{f}\left(\mathbf{x}_{n}\right)\right)\right) & \leq \frac{1}{m} \cdot \varphi\left(\rho\left(\mathbf{f}(\hat{\mathbf{x}}), \mathbf{f}\left(\mathbf{f}\left(\mathbf{x}_{n}\right)\right)\right)\right) \cdot \rho\left(\mathbf{f}(\hat{\mathbf{x}}), \mathbf{f}\left(\mathbf{f}\left(\mathbf{x}_{n}\right)\right)\right) \quad(\text { by }(22)) \\
& <\frac{1}{m} \cdot \rho\left(\mathbf{f}(\hat{\mathbf{x}}), \mathbf{f}\left(\mathbf{f}\left(\mathbf{x}_{n}\right)\right)\right) \leq \frac{1}{m} \cdot \sum_{k=1}^{m} d\left(f_{k}(\hat{\mathbf{x}}), f_{k}\left(\mathbf{f}\left(\mathbf{x}_{n}\right)\right)\right) \quad(\text { by }(21)) \\
& <\frac{\epsilon}{2} \quad(\text { by }(23)) .
\end{aligned}
$$

Since $\mathbf{F}$ and $\mathbf{f}$ are commutative, we have $\mathbf{f}\left(\mathbf{F}^{p}(\mathbf{x})\right)=\mathbf{F}^{p}(\mathbf{f}(\mathbf{x}))$ for all $\mathbf{x} \in X^{m}$, which also implies

$$
f_{k}\left(\mathbf{f}\left(\mathbf{x}_{n}\right)\right)=f_{k}\left(\mathbf{F}^{p}\left(\mathbf{x}_{n-1}\right)\right)=F_{k}^{p}\left(\mathbf{f}\left(\mathbf{x}_{n-1}\right)\right) .
$$

Now, we obtain

$$
\begin{aligned}
d\left(F_{k}^{p}(\hat{\mathbf{x}}), f_{k}(\hat{\mathbf{x}})\right) & \leq d\left(F_{k}^{p}(\hat{\mathbf{x}}), F_{k}^{p}\left(\mathbf{f}\left(\mathbf{x}_{n_{0}}\right)\right)\right)+d\left(F_{k}^{p}\left(\mathbf{f}\left(\mathbf{x}_{n_{0}}\right)\right), f_{k}(\hat{\mathbf{x}})\right) \\
& =d\left(F_{k}^{p}(\hat{\mathbf{x}}), F_{k}^{p}\left(\mathbf{f}\left(\mathbf{x}_{n_{0}}\right)\right)\right)+d\left(f_{k}\left(\mathbf{f}\left(\mathbf{x}_{n_{0}+1}\right)\right), f_{k}(\hat{\mathbf{x}})\right) \\
& <\frac{\epsilon}{2}+\frac{\epsilon}{2} \quad(\text { by }(23) \text { and }(24)) \\
& =\epsilon .
\end{aligned}
$$


Since $\epsilon$ is any positive number, we conclude that $d\left(F_{k}^{p}(\hat{\mathbf{x}}), f_{k}(\hat{\mathbf{x}})\right)=0$, which says that $F_{k}^{p}(\hat{\mathbf{x}})=$ $f_{k}(\hat{\mathbf{x}})$ for all $k=1, \ldots, m$, i.e., $\mathbf{F}^{p}(\hat{\mathbf{x}})=\mathbf{f}(\hat{\mathbf{x}})$. This proves part (i).

To prove part (ii), since $\mathbf{f}$ has the $\preceq$-comparable property, it follows that $\mathbf{f}(\hat{\mathbf{x}})$ and $\mathbf{f}(\hat{\mathbf{y}})$ are $\preceq$-mixed comparable. If $f_{k}(\hat{\mathbf{x}}) \neq f_{k}(\hat{\mathbf{y}})$, i.e., $d\left(f_{k}(\hat{\mathbf{x}}), f_{k}(\hat{\mathbf{y}})\right) \neq 0$ for some $k$, then we obtain

$$
\begin{aligned}
0 & \neq \sum_{k=1}^{m} d\left(f_{k}(\hat{\mathbf{x}}), f_{k}(\hat{\mathbf{y}})\right)=\sum_{k=1}^{m} d\left(F_{k}^{p}(\hat{\mathbf{x}}), F_{k}^{p}(\hat{\mathbf{y}})\right) \\
& \leq \frac{1}{m} \cdot \sum_{k=1}^{m} \varphi(\rho(\mathbf{f}(\hat{\mathbf{x}}), \mathbf{f}(\hat{\mathbf{y}}))) \cdot \rho(\mathbf{f}(\hat{\mathbf{x}}), \mathbf{f}(\hat{\mathbf{y}})) \quad(\text { by }(22)) \\
& <\rho(\mathbf{f}(\hat{\mathbf{x}}), \mathbf{f}(\hat{\mathbf{y}})) \leq \sum_{k=1}^{m} d\left(f_{k}(\hat{\mathbf{x}}), f_{k}(\hat{\mathbf{y}})\right) \quad(\text { by }(21)) .
\end{aligned}
$$

This contradiction says that $f_{k}(\hat{\mathbf{x}})=f_{k}(\hat{\mathbf{y}})$ for all $k=1, \ldots, m$, i.e., $\mathbf{f}(\hat{\mathbf{x}})=\mathbf{f}(\hat{\mathbf{y}})$.

To prove part (iii), using the commutativity of $\mathbf{F}$ and $\mathbf{f}$, we have

$$
\mathbf{f}(\mathbf{F}(\hat{\mathbf{x}}))=\mathbf{F}(\mathbf{f}(\hat{\mathbf{x}}))=\mathbf{F}\left(\mathbf{F}^{p}(\hat{\mathbf{x}})\right)=\mathbf{F}^{p}(\mathbf{F}(\hat{\mathbf{x}}))
$$

By taking $\hat{\mathbf{y}}=\mathbf{F}(\hat{\mathbf{x}})$, equalities (25) say that $\mathbf{f}(\hat{\mathbf{y}})=\mathbf{F}^{p}(\hat{\mathbf{y}})$. Since $\hat{\mathbf{x}}$ and $\hat{\mathbf{y}}=\mathbf{F}(\hat{\mathbf{x}})$ are $\preceq$-mixed comparable by the assumption, part (ii) says that

$$
\mathbf{f}(\hat{\mathbf{x}})=\mathbf{f}(\hat{\mathbf{y}})=\mathbf{f}(\mathbf{F}(\hat{\mathbf{x}}))=\mathbf{F}(\mathbf{f}(\hat{\mathbf{x}}))
$$

which says that $\mathbf{f}(\hat{\mathbf{x}})$ is a fixed point of $\mathbf{F}$. Given any $q \in \mathbb{N}$, we have

$$
\begin{aligned}
\mathbf{F}\left(\mathbf{f}^{q}(\hat{\mathbf{x}})\right) & =\mathbf{f}^{q-1}(\mathbf{F}(\mathbf{f}(\hat{\mathbf{x}}))) \quad(\text { by the commutativity of } \mathbf{F} \text { and } \mathbf{f}) \\
& =\mathbf{f}^{q-1}(\mathbf{f}(\hat{\mathbf{x}}))=\mathbf{f}^{q}(\hat{\mathbf{x}}),
\end{aligned}
$$

which says that $\mathbf{f}^{q}(\hat{\mathbf{x}})$ is a fixed point of $\mathbf{F}$. This completes the proof.

Theorem 2.22 Suppose that the quasi-ordered metric space $(X, d, \preceq)$ is mixed-monotonically complete and preserves the mixed-monotone convergence. Assume that the metrics $\mathfrak{d}$ and $d$ are compatible in the sense of preserving convergence. Let $I$ and $J$ be any disjoint pair of $\{1,2, \ldots, m\}$. Consider the functions $\mathbf{F}:\left(X^{m}, \mathfrak{d}, \preccurlyeq_{I}\right) \rightarrow\left(X^{m}, \mathfrak{d}, \preccurlyeq_{I}\right)$ and $\mathbf{f}:\left(X^{m}, \mathfrak{d}, \preccurlyeq_{I}\right) \rightarrow$ $\left(X^{m}, \mathfrak{d}, \preccurlyeq_{I}\right)$ satisfying $\mathbf{F}^{p}\left(X^{m}\right) \subseteq \mathbf{f}\left(X^{m}\right)$ for some $p \in \mathbb{N}$. Let $\mathbf{x}_{0}$ be a mixed $\preccurlyeq_{I}$-monotone seed element in $X^{m}$. Assume that the functions $\mathbf{F}$ and $\mathbf{f}$ satisfy the following conditions:

- $\mathbf{F}$ and $\mathbf{f}$ are commutative;

- $\mathbf{f}$ has the sequentially mixed $\preccurlyeq$-monotone property or the sequentially mixed $\preceq$-monotone property;

- $\mathbf{f}$ has the $\preccurlyeq I^{\circ}$-comparable property for any disjoint pair $I^{\circ}$ and $J^{\circ}$ of $\{1, \ldots, m\}$;

- each $f_{k}$ is continuous on $X^{m}$ for $k=1, \ldots, m$.

Suppose that there exist a function $\rho: X^{m} \times X^{m} \rightarrow \mathbb{R}_{+}$and a function of contractive factor $\varphi:[0, \infty) \rightarrow[0,1)$ such that, for any $\mathbf{x}, \mathbf{y} \in X^{m}$ and any disjoint pair $I^{\circ}$ and $J^{\circ}$ of $\{1, \ldots, m\}$ with $\mathbf{y} \preccurlyeq I^{\circ} \mathbf{x}$ or $\mathbf{x} \preccurlyeq I_{I^{\circ}} \mathbf{y}$, the following inequalities are satisfied:

$$
\rho(\mathbf{x}, \mathbf{y}) \leq \sum_{k=1}^{m} d\left(x^{(k)}, y^{(k)}\right)
$$


and, for each $k=1, \ldots, m$,

$$
d\left(F_{k}^{p}(\mathbf{x}), F_{k}^{p}(\mathbf{y})\right) \leq \frac{1}{m} \cdot \varphi(\rho(\mathbf{f}(\mathbf{x}), \mathbf{f}(\mathbf{y}))) \cdot \rho(\mathbf{f}(\mathbf{x}), \mathbf{f}(\mathbf{y}))
$$

Then the following statements hold true.

(i) There exists $\hat{\mathbf{x}} \in X^{m}$ of $\mathbf{F}$ such that $\mathbf{F}^{p}(\hat{\mathbf{x}})=\mathbf{f}(\hat{\mathbf{x}})$. If $p=1$, then $\hat{\mathbf{x}}$ is a coincidence point of $\mathbf{F}$ and $\mathbf{f}$.

(ii) If there exist a disjoint pair $I^{\circ}$ and $J^{\circ}$ of $\{1, \ldots, m\}$ and $\hat{\mathbf{y}} \in X^{m} \operatorname{such}$ that $\mathbf{F}^{p}(\hat{\mathbf{y}})=\mathbf{f}(\hat{\mathbf{y}})$ and that $\hat{\mathbf{x}}$ and $\hat{\mathbf{y}}$ are comparable with respect to the quasi-order ' $\preccurlyeq I^{\circ}$ ', then $\mathbf{f}(\hat{\mathbf{x}})=\mathbf{f}(\hat{\mathbf{y}})$.

(iii) Suppose that $\hat{\mathbf{x}}$ is obtained from part (i). If there exists a disjoint pair $I^{\circ}$ and $J^{\circ}$ of $\{1, \ldots, m\}$ such that $\hat{\mathbf{x}}$ and $\mathbf{F}(\hat{\mathbf{x}})$ are comparable with respect to the quasi-order ' $\preccurlyeq I^{\circ}$ ', then $\mathbf{f}^{q}(\hat{\mathbf{x}})$ is a fixed point of $\mathbf{F}$ for any $q \in \mathbb{N}$.

Moreover, each component $\hat{x}^{(k)}$ of $\hat{\mathbf{x}}$ is the limit of the sequence $\left\{f_{k}\left(\mathbf{x}_{n}\right)\right\}_{n \in \mathbb{N}}$ constructed in (3) for all $k=1, \ldots, m$.

Proof From the proof of Theorem 2.16, we can construct a sequence $\left\{\mathbf{x}_{n}\right\}_{n \in \mathbb{N}}$ in $X^{m}$ such that $f_{k}\left(\mathbf{x}_{n}\right) \rightarrow \hat{x}^{(k)}$ and $f_{k}\left(\mathbf{f}\left(\mathbf{x}_{n}\right)\right) \rightarrow f_{k}(\hat{\mathbf{x}})$ as $n \rightarrow \infty$, where $\left\{f_{k}\left(\mathbf{x}_{n}\right)\right\}_{n \in \mathbb{N}}$ is a mixed $\preceq$-monotone sequence for all $k=1, \ldots, m$. Since $f_{k}\left(\mathbf{f}\left(\mathbf{x}_{n}\right)\right) \rightarrow f_{k}(\hat{\mathbf{x}})$ as $n \rightarrow \infty$, given any $\epsilon>0$, there exists $n_{0} \in \mathbb{N}$ such that

$$
d\left(f_{k}\left(\mathbf{f}\left(\mathbf{x}_{n}\right)\right), f_{k}(\hat{\mathbf{x}})\right)<\frac{\epsilon}{2}
$$

for all $n \in \mathbb{N}$ with $n \geq n_{0}$ and for all $k=1, \ldots, m$. Since $\left\{f_{k}\left(\mathbf{x}_{n}\right)\right\}_{n \in \mathbb{N}}$ is a mixed $\preceq$-monotone convergent sequence for all $k=1, \ldots, m$, from observation (b) of Remark 2.19, we see that, for each $n \in \mathbb{N}$, there exists a subset $I_{n}$ of $\{1, \ldots, m\}$ such that

$$
\mathbf{f}\left(\mathbf{x}_{n}\right) \preccurlyeq I_{n} \hat{\mathbf{x}} \quad \text { or } \quad \hat{\mathbf{x}} \preccurlyeq I_{n} \mathbf{f}\left(\mathbf{x}_{n}\right) \text {. }
$$

Since $\mathbf{f}$ has the $\preccurlyeq_{I^{\circ}}$-comparable property for any subset $I^{\circ}$ of $\{1, \ldots, m\}$, it follows that

$$
\mathbf{f}\left(\mathbf{f}\left(\mathbf{x}_{n}\right)\right) \preccurlyeq I_{n} \mathbf{f}(\hat{\mathbf{x}}) \quad \text { or } \quad \mathbf{f}(\hat{\mathbf{x}}) \preccurlyeq I_{n} \mathbf{f}\left(\mathbf{f}\left(\mathbf{x}_{n}\right)\right) \text {. }
$$

For each $n \geq n_{0}$, we obtain

$$
\begin{aligned}
d & \left(F_{k}^{p}(\hat{\mathbf{x}}), F_{k}^{p}\left(\mathbf{f}\left(\mathbf{x}_{n}\right)\right)\right) \\
& \leq \frac{1}{m} \cdot \varphi\left(\rho\left(\mathbf{f}(\hat{\mathbf{x}}), \mathbf{f}\left(\mathbf{f}\left(\mathbf{x}_{n}\right)\right)\right)\right) \cdot \rho\left(\mathbf{f}(\hat{\mathbf{x}}), \mathbf{f}\left(\mathbf{f}\left(\mathbf{x}_{n}\right)\right)\right) \quad(\text { by }(29) \text { and }(27)) \\
& <\frac{1}{m} \cdot \rho\left(\mathbf{f}(\hat{\mathbf{x}}), \mathbf{f}\left(\mathbf{f}\left(\mathbf{x}_{n}\right)\right)\right) \leq \frac{1}{m} \cdot \sum_{k=1}^{m} d\left(f_{k}(\hat{\mathbf{x}}), f_{k}\left(\mathbf{f}\left(\mathbf{x}_{n}\right)\right)\right) \quad(\text { by }(30) \text { and }(26)) \\
& <\frac{\epsilon}{2} \quad(\text { by }(28)) .
\end{aligned}
$$

The remaining proof follows from a similar argument in the proof of Theorem 2.21, and the proof is complete. 
Remark 2.23 Suppose that inequalities (21) and (22) in Theorem 2.21, and that inequalities (26) and (27) in Theorem 2.22 are satisfied for any $\mathbf{x}, \mathbf{y} \in X^{m}$. Then, from the proofs of Theorems 2.21 and 2.22, we can see that parts (ii) and (iii) can be changed as follows.

(ii) $^{\prime} \quad$ If there exists $\hat{\mathbf{y}} \in X^{m}$ such that $\mathbf{F}^{p}(\hat{\mathbf{y}})=\mathbf{f}(\hat{\mathbf{y}})$, then $\mathbf{f}(\hat{\mathbf{x}})=\mathbf{f}(\hat{\mathbf{y}})$.

(iii) $^{\prime}$ Suppose that $\hat{\mathbf{x}}$ is obtained from part (i). Then $\mathbf{f}^{q}(\hat{\mathbf{x}})$ is a fixed point of $\mathbf{F}$ for any $q \in \mathbb{N}$.

The assumption that $\mathbf{f}$ has the $\preccurlyeq I^{\circ}$-comparable property for any disjoint pair $I^{\circ}$ and $J^{\circ}$ of $\{1, \ldots, m\}$ in Theorem 2.22 can be dropped by strengthening inequalities (26) as shown below.

Theorem 2.24 Suppose that the quasi-ordered metric space $(X, d, \preceq)$ is mixed-monotonically complete and preserves the mixed-monotone convergence. Assume that the metrics $\mathfrak{d}$ and $d$ are compatible in the sense of preserving convergence. Consider the functions $\mathbf{F}$ : $\left(X^{m}, \mathfrak{d}, \preccurlyeq_{I}\right) \rightarrow\left(X^{m}, \mathfrak{d}, \preccurlyeq_{I}\right)$ and $\mathbf{f}:\left(X^{m}, \mathfrak{d}, \preccurlyeq_{I}\right) \rightarrow\left(X^{m}, \mathfrak{d}, \preccurlyeq_{I}\right)$ satisfying $\mathbf{F}^{p}\left(X^{m}\right) \subseteq \mathbf{f}\left(X^{m}\right)$ for some $p \in \mathbb{N}$. Let $\mathbf{x}_{0}$ be a mixed $\preccurlyeq_{I}$-monotone seed element in $X^{m}$. Assume that the functions $\mathbf{F}$ and $\mathbf{f}$ satisfy the following conditions:

- $\mathbf{F}$ and $\mathbf{f}$ are commutative;

- $\mathbf{f}$ has the sequentially mixed $\preccurlyeq_{I}$-monotone property or the sequentially mixed $\preceq$-monotone property;

- each $f_{k}$ is continuous on $X^{m}$ for $k=1, \ldots, m$.

Suppose that there exists a function $\rho: X^{m} \times X^{m} \rightarrow \mathbb{R}_{+}$such that, for any $\mathbf{x}, \mathbf{y} \in X^{m}$, the following inequality is satisfied:

$$
\rho(\mathbf{x}, \mathbf{y}) \leq \sum_{k=1}^{m} d\left(x^{(k)}, y^{(k)}\right),
$$

and that there exists a function of contractive factor $\varphi:[0, \infty) \rightarrow[0,1)$ such that, for any $\mathbf{x}, \mathbf{y} \in X^{m}$ and any disjoint pair $I^{\circ}$ and $J^{\circ}$ of $\{1, \ldots, m\}$ with $\mathbf{y} \preccurlyeq I^{\circ} \mathbf{x}$ or $\mathbf{x} \preccurlyeq I^{\circ} \mathbf{y}$, the following inequality is satisfied:

$$
d\left(F_{k}^{p}(\mathbf{x}), F_{k}^{p}(\mathbf{y})\right) \leq \frac{1}{m} \cdot \varphi(\rho(\mathbf{f}(\mathbf{x}), \mathbf{f}(\mathbf{y}))) \cdot \rho(\mathbf{f}(\mathbf{x}), \mathbf{f}(\mathbf{y})) \quad \text { for each } k=1, \ldots, m .
$$

Then the following statements hold true.

(i) There exists $\hat{\mathbf{x}} \in X^{m}$ of $\mathbf{F}$ such that $\mathbf{F}^{p}(\hat{\mathbf{x}})=\mathbf{f}(\hat{\mathbf{x}})$. If $p=1$, then $\hat{\mathbf{x}}$ is a coincidence point of $\mathbf{F}$ and $\mathbf{f}$.

(ii) If there exist a disjoint pair $I^{\circ}$ and $J^{\circ}$ of $\{1, \ldots, m\}$ and $\hat{\mathbf{y}} \in X^{m}$ such that $\mathbf{F}^{p}(\hat{\mathbf{y}})=\mathbf{f}(\hat{\mathbf{y}})$ and that $\hat{\mathbf{x}}$ and $\hat{\mathbf{y}}$ are comparable with respect to the quasi-order ' $\preccurlyeq I^{\circ}$ ', then $\mathbf{f}(\hat{\mathbf{x}})=\mathbf{f}(\hat{\mathbf{y}})$.

(iii) Suppose that $\hat{\mathbf{x}}$ is obtained from part (i). If there exists a disjoint pair $I^{\circ}$ and $J^{\circ}$ of $\{1, \ldots, m\}$ such that $\hat{\mathbf{x}}$ and $\mathbf{F}(\hat{\mathbf{x}})$ are comparable with respect to the quasi-order ' $\preccurlyeq I^{\circ}$, then $\mathbf{f}^{q}(\hat{\mathbf{x}})$ is a fixed point of $\mathbf{F}$ for any $q \in \mathbb{N}$.

Moreover, each component $\hat{x}^{(k)}$ of $\hat{\mathbf{x}}$ is the limit of the sequence $\left\{f_{k}\left(\mathbf{x}_{n}\right)\right\}_{n \in \mathbb{N}}$ constructed in (3) for all $k=1, \ldots, m$.

Proof Since inequalities (31) are satisfied for any $\mathbf{x}$ and $\mathbf{y}$, the arguments in the proof of Theorem 2.22 are still valid without considering (30). This completes the proof. 
Next, we shall consider the uniqueness for a common fixed point in the $\preceq$-mixed comparable sense.

Definition 2.25 Let $(X, \preceq)$ be a quasi-ordered set. Consider the functions $\mathbf{F}: X^{m} \rightarrow X^{m}$ and $\mathbf{f}: X^{m} \rightarrow X^{m}$ defined on the product set $X^{m}$ into itself. The common fixed point $\hat{\mathbf{x}} \in$ $X^{m}$ of $\mathbf{F}$ and $\mathbf{f}$ is unique in the $\preceq$-mixed comparable sense if and only if, for any other common fixed point $\mathbf{x}$ of $\mathbf{F}$ and $\mathbf{f}$, if $\mathbf{x}$ and $\hat{\mathbf{x}}$ are $\preceq$-mixed comparable, then $\mathbf{x}=\hat{\mathbf{x}}$.

Theorem 2.26 Suppose that the quasi-ordered metric space $(X, d, \preceq)$ is mixed-monotonically complete and preserves the mixed-monotone convergence. Assume that the metrics $\mathfrak{d}$ and $d$ are compatible in the sense of preserving continuity. Consider the functions $\mathbf{F}$ : $\left(X^{m}, \mathfrak{d}\right) \rightarrow\left(X^{m}, \mathfrak{d}\right)$ and $\mathbf{f}:\left(X^{m}, \mathfrak{d}\right) \rightarrow\left(X^{m}, \mathfrak{d}\right)$ satisfying $\mathbf{F}^{p}\left(X^{m}\right) \subseteq \mathbf{f}\left(X^{m}\right)$ for some $p \in \mathbb{N}$. Let $\mathbf{x}_{0}$ be a mixed $\preceq$-monotone seed element in $X^{m}$. Assume that the functions $\mathbf{F}$ and $\mathbf{f}$ satisfy the following conditions:

- $\mathbf{F}$ and $\mathbf{f}$ are commutative;

- $\mathbf{f}$ has the $\preceq$-comparable property and the sequentially mixed $\preceq$-monotone property;

- $\mathbf{F}^{p}$ is continuous with respect to $\mathbf{f}$ on $X^{m}$;

- each $f_{k}$ is continuous on $X^{m}$ for $k=1, \ldots, m$.

Suppose that there exist a function $\rho: X^{m} \times X^{m} \rightarrow \mathbb{R}_{+}$and a function of contractive factor $\varphi:[0, \infty) \rightarrow[0,1)$ such that, for any two $\preceq$-mixed comparable elements $\mathbf{x}$ and $\mathbf{y}$ in $X^{m}$, the following inequalities are satisfied:

$$
\rho(\mathbf{x}, \mathbf{y}) \leq \sum_{k=1}^{m} d\left(x^{(k)}, y^{(k)}\right)
$$

and, for each $k=1, \ldots, m$,

$$
d\left(F_{k}^{p}(\mathbf{x}), F_{k}^{p}(\mathbf{y})\right) \leq \frac{1}{m} \cdot \varphi(\rho(\mathbf{f}(\mathbf{x}), \mathbf{f}(\mathbf{y}))) \cdot \rho(\mathbf{f}(\mathbf{x}), \mathbf{f}(\mathbf{y})) .
$$

Then the following statements hold true.

(i) $\mathbf{F}^{p}$ and $\mathbf{f}$ have a unique common fixed point $\hat{\mathbf{x}}$ in the $\preceq$-mixed comparable sense. Equivalently, if $\hat{\mathbf{y}}$ is another common fixed point of $\mathbf{F}^{p}$ and $\mathbf{f}$, and is $\preceq$-mixed comparable with $\hat{\mathbf{x}}$, then $\hat{\mathbf{y}}=\hat{\mathbf{x}}$.

(ii) For $p \neq 1$, suppose that $\mathbf{F}(\hat{\mathbf{x}})$ and $\hat{\mathbf{x}}$ obtained in (i) are $\preceq$-mixed comparable. Then $\mathbf{F}$ and $\mathbf{f}$ have a unique common fixed point $\hat{\mathbf{x}}$ in the $\preceq$-mixed comparable sense.

Moreover, each component $\hat{x}^{(k)}$ of $\hat{\mathbf{x}}$ is the limit of the sequence $\left\{f_{k}\left(\mathbf{x}_{n}\right)\right\}_{n \in \mathbb{N}}$ constructed in (3) for all $k=1, \ldots, m$.

Proof To prove part (i), from Proposition 2.8 and part (i) of Theorem 2.21, we have $\mathbf{f}(\hat{\mathbf{x}})=$ $\mathbf{F}^{p}(\hat{\mathbf{x}})$. From Theorem 2.14 , we also have $\mathbf{F}^{p}(\hat{\mathbf{x}})=\hat{\mathbf{x}}$. Therefore, we obtain

$$
\hat{\mathbf{x}}=\mathbf{f}(\hat{\mathbf{x}})=\mathbf{F}^{p}(\hat{\mathbf{x}}) .
$$

This shows that $\hat{\mathbf{x}}$ is a common fixed point of $\mathbf{F}^{p}$ and $\mathbf{f}$. For the uniqueness in the $\preceq$-mixed comparable sense, let $\hat{\mathbf{y}}$ be another common fixed point of $\mathbf{F}^{p}$ and $\mathbf{f}$ such that $\hat{\mathbf{y}}$ and $\hat{\mathbf{x}}$ are 
$\preceq$-mixed comparable, i.e., $\hat{\mathbf{y}}=\mathbf{f}(\hat{\mathbf{y}})=\mathbf{F}^{p}(\hat{\mathbf{y}})$. By part (ii) of Theorem 2.21, we have $\mathbf{f}(\hat{\mathbf{x}})=\mathbf{f}(\hat{\mathbf{y}})$. Therefore, by the triangle inequality, we obtain

$$
d(\hat{\mathbf{x}}, \hat{\mathbf{y}}) \leq d(\hat{\mathbf{x}}, \mathbf{f}(\hat{\mathbf{x}}))+d(\mathbf{f}(\hat{\mathbf{x}}), \mathbf{f}(\hat{\mathbf{y}}))+d(\mathbf{f}(\hat{\mathbf{y}}), \hat{\mathbf{y}})=0,
$$

which says that $\hat{\mathbf{x}}=\hat{\mathbf{y}}$. This proves part (i).

To prove part (ii), since $\mathbf{F}(\hat{\mathbf{x}})$ and $\hat{\mathbf{x}}$ are $\preceq$-mixed comparable, part (iii) of Theorem 2.21 says that $\mathbf{f}(\hat{\mathbf{x}})$ is a fixed point of $\mathbf{F}$, i.e., $\mathbf{f}(\hat{\mathbf{x}})=\mathbf{F}(\mathbf{f}(\hat{\mathbf{x}}))$, which implies $\hat{\mathbf{x}}=\mathbf{F}(\hat{\mathbf{x}})$, since $\hat{\mathbf{x}}=\mathbf{f}(\hat{\mathbf{x}})$. This shows that $\hat{\mathbf{x}}$ is a common fixed point of $\mathbf{F}$ and $\mathbf{f}$. For the uniqueness in the $\preceq$-mixed comparable sense, let $\hat{\mathbf{y}}$ be another common fixed point of $\mathbf{F}$ and $\mathbf{f}$ such that $\hat{\mathbf{y}}$ and $\hat{\mathbf{x}}$ are $\preceq$-mixed comparable, i.e., $\hat{\mathbf{y}}=\mathbf{f}(\hat{\mathbf{y}})=\mathbf{F}(\hat{\mathbf{y}})$. Then we have

$$
\hat{\mathbf{y}}=\mathbf{f}(\hat{\mathbf{y}})=\mathbf{F}(\hat{\mathbf{y}})=\mathbf{F}(\mathbf{f}(\hat{\mathbf{y}}))=\mathbf{F}^{2}(\hat{\mathbf{y}})=\cdots=\mathbf{F}^{p}(\hat{\mathbf{y}})
$$

By part (ii) of Theorem 2.21, we have $\mathbf{f}(\hat{\mathbf{x}})=\mathbf{f}(\hat{\mathbf{y}})$. From (34), we can similarly obtain $\hat{\mathbf{x}}=\hat{\mathbf{y}}$. This completes the proof.

Since we consider a metric space $(X, d, \preceq)$ endowed with a quasi-order ' $\leq$ ', given any disjoint pair $I$ and $J$ of $\{1, \ldots, p\}$, we can define a quasi-order ' $\preccurlyeq_{I}$ ' on $X^{m}$ as given in (1). Now, given any $\mathbf{x} \in X^{m}$, we define the chain $\mathfrak{C}\left(\preccurlyeq_{I}, \mathbf{x}\right)$ containing $\mathbf{x}$ as follows:

$$
\begin{aligned}
\mathfrak{C}\left(\preccurlyeq_{I}, \mathbf{x}\right) & =\left\{\mathbf{y} \in X^{m}: \mathbf{y} \preccurlyeq_{I} \mathbf{x} \text { or } \mathbf{x} \preccurlyeq_{I} \mathbf{y}\right\} \\
& =\left\{\mathbf{y} \in X^{m}: \mathbf{x} \text { and } \mathbf{y} \text { are comparable with respect to ' } \preccurlyeq_{I} \text { ' }\right\} .
\end{aligned}
$$

Next, we shall introduce the concept of chain-uniqueness for a common fixed point.

Definition 2.27 Let $(X, \preceq)$ be a quasi-ordered set. Consider the functions $\mathbf{F}: X^{m} \rightarrow X^{m}$ and $\mathbf{f}: X^{m} \rightarrow X^{m}$ defined on the product set $X^{m}$ into itself. The common fixed point $\hat{\mathbf{x}} \in$ $X^{m}$ of $\mathbf{F}$ and $\mathbf{f}$ is called chain-unique if and only if, given any other common fixed point $\mathbf{x}$ of $\mathbf{F}$ and $\mathbf{f}$, if $\mathbf{x} \in \mathfrak{C}\left(\preccurlyeq I^{\circ}, \hat{\mathbf{x}}\right)$ for some disjoint pair $I^{\circ}$ and $J^{\circ}$ of $\{1, \ldots, m\}$, then $\mathbf{x}=\hat{\mathbf{x}}$.

Theorem 2.28 Suppose that the quasi-ordered metric space $(X, d, \preceq)$ is mixed-monotonically complete and preserves the mixed-monotone convergence. Assume that the metrics $\mathfrak{d}$ and $d$ are compatible in the sense of preserving continuity. Consider the functions $\mathbf{F}$ : $\left(X^{m}, \mathfrak{d}, \preccurlyeq_{I}\right) \rightarrow\left(X^{m}, \mathfrak{d}, \preccurlyeq_{I}\right)$ and $\mathbf{f}:\left(X^{m}, \mathfrak{d}, \preccurlyeq_{I}\right) \rightarrow\left(X^{m}, \mathfrak{d}, \preccurlyeq_{I}\right)$ satisfying $\mathbf{F}^{p}\left(X^{m}\right) \subseteq \mathbf{f}\left(X^{m}\right)$ for some $p \in \mathbb{N}$. Let $\mathbf{x}_{0}$ be a mixed $\preccurlyeq_{I}$-monotone seed element in $X^{m}$. Assume that the functions $\mathbf{F}$ and $\mathbf{f}$ satisfy the following conditions:

- $\mathbf{F}$ and $\mathbf{f}$ are commutative;

- f has the sequentially mixed $\preccurlyeq I_{\text {-monotone property or the sequentially mixed }}$ $\preceq$-monotone property;

- $\mathbf{F}^{p}$ is continuous with respect to $\mathbf{f}$ on $X^{m}$;

- each $f_{k}$ is continuous on $X^{m}$ for $k=1, \ldots, m$.

Suppose that there exist a function $\rho: X^{m} \times X^{m} \rightarrow \mathbb{R}_{+}$and a function of contractive factor $\varphi:[0, \infty) \rightarrow[0,1)$ such that, for any $\mathbf{x}, \mathbf{y} \in X^{m}$ and any disjoint pair $I^{\circ}$ and $J^{\circ}$ of $\{1, \ldots, m\}$ with $\mathbf{y} \preccurlyeq I^{\circ} \mathbf{x}$ or $\mathbf{x} \preccurlyeq I_{I^{\circ}} \mathbf{y}$, the following inequalities are satisfied:

$$
\rho(\mathbf{x}, \mathbf{y}) \leq \sum_{k=1}^{m} d\left(x^{(k)}, y^{(k)}\right)
$$


and, for each $k=1, \ldots, m$,

$$
d\left(F_{k}^{p}(\mathbf{x}), F_{k}^{p}(\mathbf{y})\right) \leq \frac{1}{m} \cdot \varphi(\rho(\mathbf{f}(\mathbf{x}), \mathbf{f}(\mathbf{y}))) \cdot \rho(\mathbf{f}(\mathbf{x}), \mathbf{f}(\mathbf{y})) .
$$

Then the following statements hold true.

(i) $\mathbf{F}^{p}$ and $\mathbf{f}$ have a chain-unique common fixed point $\hat{\mathbf{x}}$. Equivalently, if $\hat{\mathbf{y}} \in \mathfrak{C}\left(\preccurlyeq I^{\circ}, \hat{\mathbf{x}}\right)$ is another common fixed point of $\mathbf{F}^{p}$ and $\mathbf{f}$ for some disjoint pair $I^{\circ}$ and $J^{\circ}$ of $\{1, \ldots, m\}$, then $\hat{\mathbf{y}}=\hat{\mathbf{x}}$.

(ii) For $p \neq 1$, suppose that $\mathbf{F}(\hat{\mathbf{x}})$ and $\hat{\mathbf{x}}$ obtained in (i) are comparable with respect to the quasi-order ' $\preccurlyeq I^{\circ}$ ' for some disjoint pair $I^{\circ}$ and $J^{\circ}$ of $\{1, \ldots, m\}$. Then $\mathbf{F}$ and $\mathbf{f}$ have a chain-unique common fixed point $\hat{\mathbf{x}}$.

Moreover, each component $\hat{x}^{(k)}$ of $\hat{\mathbf{x}}$ is the limit of the sequence $\left\{f_{k}\left(\mathbf{x}_{n}\right)\right\}_{n \in \mathbb{N}}$ constructed in (3) for all $k=1, \ldots, m$.

Proof To prove part (i), from Proposition 2.8 and part (i) of Theorem 2.22, we can show that $\hat{\mathbf{x}}$ is a common fixed point of $\mathbf{F}^{p}$ and $\mathbf{f}$. For the chain-uniqueness, let $\hat{\mathbf{y}}$ be another common fixed point of $\mathbf{F}^{p}$ and $\mathbf{f}$ with $\hat{\mathbf{y}} \preccurlyeq I_{I^{\circ}} \hat{\mathbf{x}}$ or $\hat{\mathbf{x}} \preccurlyeq I^{\circ} \hat{\mathbf{y}}$ for some disjoint pair $I^{\circ}$ and $J^{\circ}$ of $\{1, \ldots, m\}$, i.e., $\hat{\mathbf{y}}=\mathbf{f}(\hat{\mathbf{y}})=\mathbf{F}^{p}(\hat{\mathbf{y}})$. By part (ii) of Theorem 2.22, we have $\mathbf{f}(\hat{\mathbf{x}})=\mathbf{f}(\hat{\mathbf{y}})$. Therefore, according to (34), we can obtain $\hat{\mathbf{x}}=\hat{\mathbf{y}}$. This proves part (i). Part (ii) can be similarly obtained by applying Theorem 2.22 to the argument in the proof of part (ii) of Theorem 2.26. This completes the proof.

Remark 2.29 We strongly assume that inequalities (32) and (33) in Theorem 2.26, and that inequalities (35) and (36) in Theorem 2.28 are satisfied for any $\mathbf{x}, \mathbf{y} \in X^{m}$. Then, from Remark 2.23 and the proofs of Theorems 2.26 and 2.28, it follows that parts (i) and (ii) can be combined together to conclude that $\mathbf{F}$ and $\mathbf{f}$ have a unique common fixed point $\hat{\mathbf{x}}$.

\section{Coincidence point theorems in the monotonically complete quasi-ordered metric space}

Now, we are going to weaken the concept of mixed-monotone completeness for the quasiordered metric space. Let $(X, d, \preceq)$ be a metric space endowed with a quasi-order ' $\preceq$ '. We say that the sequence $\left\{x_{n}\right\}_{n \in \mathbb{N}}$ in $(X, \preceq)$ is $\preceq$-increasing if and only if $x_{k} \preceq x_{k+1}$ for all $k \in \mathbb{N}$. The concept of $\preceq$-decreasing sequence can be similarly defined. The sequence $\left\{x_{n}\right\}_{n \in \mathbb{N}}$ in $(X, \preceq)$ is called $\preceq$-monotone if and only if $\left\{x_{n}\right\}_{n \in \mathbb{N}}$ is either $\preceq$-increasing or $\preceq$-decreasing.

Let $I$ and $J$ be a disjoint pair of $\{1,2, \ldots, m\}$. We say that the sequence $\left\{\mathbf{x}_{n}\right\}_{n \in \mathbb{N}}$ in $\left(X^{m}, \preccurlyeq_{I}\right)$ is $\preccurlyeq_{I}$-increasing if and only if $\mathbf{x}_{n} \preccurlyeq_{I} \mathbf{x}_{n+1}$ for all $n \in \mathbb{N}$. The concept of $\preccurlyeq_{I}$-decreasing sequence can be similarly defined. The sequence $\left\{\mathbf{x}_{n}\right\}_{n \in \mathbb{N}}$ in $\left(X^{m}, \preccurlyeq_{I}\right)$ is called $\preccurlyeq_{I}$-monotone if and only if $\left\{\mathbf{x}_{n}\right\}_{n \in \mathbb{N}}$ is either $\preccurlyeq_{I}$-increasing or $\preccurlyeq_{I}$-decreasing.

Given a disjoint pair $I$ and $J$ of $\{1,2, \ldots, m\}$, let $\mathbf{f}:\left(X^{m}, \preccurlyeq_{I}\right) \rightarrow\left(X^{m}, \preccurlyeq_{I}\right)$ be a function defined on $\left(X^{m}, \preccurlyeq_{I}\right)$ into itself. We say that $\mathbf{f}$ is $\preccurlyeq_{I}$-increasing if and only if $\mathbf{x} \preccurlyeq_{I} \mathbf{y}$ implies $\mathbf{f}(\mathbf{x}) \preccurlyeq_{I} \mathbf{f}(\mathbf{y})$. The concept of $\preccurlyeq_{I}$-decreasing function can be similarly defined. The function $\mathbf{f}$ is called $\preccurlyeq_{I}$-monotone if and only if $\mathbf{f}$ is either $\preccurlyeq_{I}$-increasing or $\preccurlyeq_{I}$-decreasing.

In the previous section, we consider the mixed $\preccurlyeq_{I}$-monotone seed element. Now, we shall consider another concept of seed element. Given a disjoint pair $I$ and $J$ of $\{1,2, \ldots, m\}$, we say that the initial element $\mathbf{x}_{0}$ is a $\preccurlyeq_{I}$-monotone seed element of $X^{m}$ if and only if the sequence $\left\{\mathbf{x}_{n}\right\}_{n \in \mathbb{N}}$ constructed from (3) is a $\preccurlyeq_{I}$-monotone sequence. It is obvious that if $\mathbf{x}_{0}$ is a $\preccurlyeq_{I}$-monotone seed element, then it is also a mixed $\preccurlyeq_{I}$-monotone seed element. 
Definition 3.1 Let $(X, d, \preceq)$ be a metric space endowed with a quasi-order ' $\preceq$ '. We say that $(X, d, \preceq)$ is monotonically complete if and only if each $\preceq$-monotone Cauchy sequence $\left\{x_{n}\right\}_{n \in \mathbb{N}}$ in $X$ is convergent.

It is obvious that if $(X, d, \preceq)$ is a mixed-monotonically complete quasi-ordered metric space, then it is also a monotonically complete quasi-ordered metric space. However, the converse is not true. In other words, the concept of monotone completeness is weaker than that of mixed-monotone completeness.

Theorem 3.2 Suppose that the quasi-ordered metric space $(X, d, \preceq)$ is monotonically complete, and that the metrics $\mathfrak{d}$ and $d$ are compatible in the sense of preserving continuity. Consider the functions $\mathbf{F}:\left(X^{m}, \mathfrak{d}, \preccurlyeq_{I}\right) \rightarrow\left(X^{m}, \mathfrak{d}, \preccurlyeq_{I}\right)$ and $\mathbf{f}:\left(X^{m}, \mathfrak{d}, \preccurlyeq_{I}\right) \rightarrow\left(X^{m}, \mathfrak{d}, \preccurlyeq_{I}\right)$ satisfying $\mathbf{F}^{p}\left(X^{m}\right) \subseteq \mathbf{f}\left(X^{m}\right)$ for some $p \in \mathbb{N}$. Let $\mathbf{x}_{0}$ be a $\preccurlyeq_{I}$-monotone seed element in $X^{m}$. Assume that the functions $\mathbf{F}$ and $\mathbf{f}$ satisfy the following conditions:

- $\mathbf{F}$ and $\mathbf{f}$ are commutative;

- $\mathbf{f}$ is $\preccurlyeq_{I}$-monotone;

- $\mathbf{F}^{p}$ is continuous with respect to $\mathbf{f}$ on $X^{m}$;

- each $f_{k}$ is continuous on $X^{m}$ for $k=1, \ldots, m$.

Suppose that there exist a function $\rho: X^{m} \times X^{m} \rightarrow \mathbb{R}_{+}$and a function of contractive factor $\varphi:[0, \infty) \rightarrow[0,1)$ such that, for any $\mathbf{x}, \mathbf{y} \in X^{m}$ with $\mathbf{y} \preccurlyeq_{I} \mathbf{x}$ or $\mathbf{x} \preccurlyeq_{I} \mathbf{y}$, the following inequalities

$$
\rho(\mathbf{x}, \mathbf{y}) \leq \sum_{k=1}^{m} d\left(x^{(k)}, y^{(k)}\right)
$$

and

$$
d\left(F_{k}^{p}(\mathbf{x}), F_{k}^{p}(\mathbf{y})\right) \leq \frac{1}{m} \cdot \varphi(\rho(\mathbf{f}(\mathbf{x}), \mathbf{f}(\mathbf{y}))) \cdot \rho(\mathbf{f}(\mathbf{x}), \mathbf{f}(\mathbf{y}))
$$

are satisfied for all $k=1, \ldots, m$. Then $\mathbf{F}^{p}$ has a fixed point $\hat{\mathbf{x}}$ such that each component $\hat{x}^{(k)}$ of $\hat{\mathbf{x}}$ is the limit of the sequence $\left\{f_{k}\left(\mathbf{x}_{n}\right)\right\}_{n \in \mathbb{N}}$ constructed in (3) for all $k=1, \ldots, m$.

Proof We consider the sequence $\left\{\mathbf{x}_{n}\right\}_{n \in \mathbb{N}}$ constructed from (3). Since $\mathbf{x}_{0}$ is a $\preccurlyeq_{I}$-monotone seed element in $X^{m}$, i.e., $\mathbf{x}_{n} \preccurlyeq_{I} \mathbf{x}_{n+1}$ for all $n \in \mathbb{N}$ or $\mathbf{x}_{n+1} \preccurlyeq_{I} \mathbf{x}_{n}$ for all $n \in \mathbb{N}$, according to inequalities (38), we obtain

$$
\begin{aligned}
d\left(f_{k}\left(\mathbf{x}_{n+1}\right), f_{k}\left(\mathbf{x}_{n}\right)\right) & =d\left(F_{k}^{p}\left(\mathbf{x}_{n}\right), F_{k}^{P}\left(\mathbf{x}_{n-1}\right)\right) \\
& \leq \frac{1}{m} \varphi\left(\rho\left(\mathbf{f}\left(\mathbf{x}_{n}\right), \mathbf{f}\left(\mathbf{x}_{n-1}\right)\right)\right) \cdot \rho\left(\mathbf{f}\left(\mathbf{x}_{n}\right), \mathbf{f}\left(\mathbf{x}_{n-1}\right)\right) .
\end{aligned}
$$

Since $\mathbf{f}$ is $\preccurlyeq_{I}$-monotone, it follows that $\mathbf{f}\left(\mathbf{x}_{n}\right) \preccurlyeq_{I} \mathbf{f}\left(\mathbf{x}_{n+1}\right)$ for all $n \in \mathbb{N}$ or $\mathbf{f}\left(\mathbf{x}_{n+1}\right) \preccurlyeq_{I} \mathbf{f}\left(\mathbf{x}_{n}\right)$ for all $n \in \mathbb{N}$. Then we have

$$
\begin{aligned}
\rho\left(\mathbf{f}\left(\mathbf{x}_{n+1}\right), \mathbf{f}\left(\mathbf{x}_{n}\right)\right) & \leq \sum_{k=1}^{m} d\left(f_{k}\left(\mathbf{x}_{n+1}\right), f_{k}\left(\mathbf{x}_{n}\right)\right) \quad(\text { by }(37)) \\
& \leq \varphi\left(\rho\left(\mathbf{f}\left(\mathbf{x}_{n}\right), \mathbf{f}\left(\mathbf{x}_{n-1}\right)\right)\right) \cdot \rho\left(\mathbf{f}\left(\mathbf{x}_{n}\right), \mathbf{f}\left(\mathbf{x}_{n-1}\right)\right) \quad(\text { by (39)) } .
\end{aligned}
$$


According to the proof of Theorem 2.14, we can show that $\left\{f_{k}\left(\mathbf{x}_{n}\right)\right\}_{n \in \mathbb{N}}$ is a Cauchy sequence in $X$ for any fixed $k=1, \ldots, n$. Since $\mathbf{f}$ is $\preccurlyeq_{I}$-monotone and $\left\{\mathbf{x}_{n}\right\}_{n \in \mathbb{N}}$ is a $\preccurlyeq_{I}$-monotone sequence, it follows that $\left\{\mathbf{f}\left(\mathbf{x}_{n}\right)\right\}_{n \in \mathbb{N}}$ is a $\preccurlyeq_{I}$-monotone sequence.

- If $\left\{\mathbf{f}\left(\mathbf{x}_{n}\right)\right\}_{n \in \mathbb{N}}$ is a $\preccurlyeq_{I}$-increasing sequence, then $\left\{f_{k}\left(\mathbf{x}_{n}\right)\right\}_{n \in \mathbb{N}}$ is a $\preceq$-increasing Cauchy sequence for $k \in I$, and is a $\preceq$-decreasing Cauchy sequence for $k \in J$.

- If $\left\{\mathbf{f}\left(\mathbf{x}_{n}\right)\right\}_{n \in \mathbb{N}}$ is a $\preccurlyeq_{I}$-decreasing sequence, then $\left\{f_{k}\left(\mathbf{x}_{n}\right)\right\}_{n \in \mathbb{N}}$ is a $\preceq$-decreasing Cauchy sequence for $k \in I$, and is a $\preceq$-increasing Cauchy sequence for $k \in J$.

By the monotone completeness of $X$, there exists $\hat{x}^{(k)} \in X$ such that $f_{k}\left(\mathbf{x}_{n}\right) \rightarrow \hat{x}^{(k)}$ as $n \rightarrow$ $\infty$ for $k=1, \ldots, m$. The remaining proof follows from the same argument in the proof of Theorem 2.14. This completes the proof.

Next, we shall study the coincidence point without considering the continuity of $\mathbf{F}^{p}$. However, we need to introduce the concept of monotone convergence given below.

Definition 3.3 Let $(X, d, \preceq)$ be a metric space endowed with a quasi-order ' $\preceq$ '. We say that $(X, d, \preceq)$ preserves the monotone convergence if and only if, for each $\preceq$-monotone sequence $\left\{x_{n}\right\}_{n \in \mathbb{N}}$ that converges to $\hat{x}$, either one of the following conditions is satisfied:

- if $\left\{x_{n}\right\}_{n \in \mathbb{N}}$ is a $\preceq$-increasing sequence, then $x_{n} \preceq \hat{x}$ for each $n \in \mathbb{N}$;

- if $\left\{x_{n}\right\}_{n \in \mathbb{N}}$ is a $\preceq$-decreasing sequence, then $\hat{x} \preceq x_{n}$ for each $n \in \mathbb{N}$.

Remark 3.4 Let $(X, d, \preceq)$ be a metric space endowed with a quasi-order ' $\preceq$ ' and preserve the monotone convergence. Given a disjoint pair $I$ and $J$ of $\{1, \ldots, m\}$, suppose that $\left\{\mathbf{x}_{n}\right\}_{n \in \mathbb{N}}$ is a $\preccurlyeq_{I}$-monotone sequence such that each sequence $\left\{x_{n}^{(k)}\right\}_{n \in \mathbb{N}}$ converges to $\hat{x}^{(k)}$ for $k=$ $1, \ldots, m$. We consider the following situation.

- If $\left\{\mathbf{x}_{n}\right\}_{n \in \mathbb{N}}$ is a $\preccurlyeq_{I}$-increasing sequence, then $\left\{x_{n}^{(k)}\right\}_{n \in \mathbb{N}}$ is a $\preceq$-increasing sequence for $k \in I$, and is a $\preceq$-decreasing sequence for $k \in J$. By the monotone convergence, we see that, for each $n \in \mathbb{N}, x_{n}^{(k)} \preceq \hat{x}^{(k)}$ for $k \in I$ and $x_{n}^{(k)} \succeq \hat{x}^{(k)}$ for $k \in J$, which shows that $\mathbf{x}_{n} \preccurlyeq_{I} \hat{\mathbf{x}}$ for all $n \in \mathbb{N}$.

- If $\left\{\mathbf{x}_{n}\right\}_{n \in \mathbb{N}}$ is a $\preccurlyeq_{I}$-decreasing sequence, then $\left\{x_{n}^{(k)}\right\}_{n \in \mathbb{N}}$ is a $\preceq$-decreasing sequence for $k \in I$, and is a $\preceq$-increasing sequence for $k \in J$. By the monotone convergence, we see that, for each $n \in \mathbb{N}, x_{n}^{(k)} \succeq \hat{x}^{(k)}$ for $k \in I$ and $x_{n}^{(k)} \preceq \hat{x}^{(k)}$ for $k \in J$, which shows that $\mathbf{x}_{n} \succcurlyeq_{I} \hat{\mathbf{x}}$ for all $n \in \mathbb{N}$.

Therefore, we conclude that $\mathbf{x}_{n}$ and $\hat{\mathbf{x}}$ are comparable with respect to ' $\preccurlyeq$ ' for all $n \in \mathbb{N}$.

Theorem 3.5 Suppose that the quasi-ordered metric space $(X, d, \preceq)$ is monotonically complete and preserves the monotone convergence. Assume that the metrics $\mathfrak{d}$ and $d$ are compatible in the sense of preserving convergence. Consider the functions $\mathbf{F}:\left(X^{m}, \mathfrak{d}, \preccurlyeq_{I}\right) \rightarrow$ $\left(X^{m}, \mathfrak{d}, \preccurlyeq_{I}\right)$ and $\mathbf{f}:\left(X^{m}, \mathfrak{d}, \preccurlyeq_{I}\right) \rightarrow\left(X^{m}, \mathfrak{d}, \preccurlyeq_{I}\right)$ satisfying $\mathbf{F}^{p}\left(X^{m}\right) \subseteq \mathbf{f}\left(X^{m}\right)$ for some $p \in \mathbb{N}$. Let $\mathbf{x}_{0}$ be $a \preccurlyeq{ }_{I}$-monotone seed element in $X^{m}$. Assume that the functions $\mathbf{F}$ and $\mathbf{f}$ satisfy the following conditions:

- $\mathbf{F}$ and $\mathbf{f}$ are commutative;

- $\mathbf{f}$ is $\preccurlyeq_{I^{-} \text {monotone; }}$

- each $f_{k}$ is continuous on $X^{m}$ for $k=1, \ldots, m$.

Suppose that there exist a function $\rho: X^{m} \times X^{m} \rightarrow \mathbb{R}_{+}$and a function of contractive factor $\varphi:[0, \infty) \rightarrow[0,1)$ such that, for any $\mathbf{x}, \mathbf{y} \in X^{m}$ with $\mathbf{y} \preccurlyeq_{I} \mathbf{x}$ or $\mathbf{x} \preccurlyeq_{I} \mathbf{y}$, the following inequalities

$$
\rho(\mathbf{x}, \mathbf{y}) \leq \sum_{k=1}^{m} d\left(x^{(k)}, y^{(k)}\right)
$$


and

$$
d\left(F_{k}^{p}(\mathbf{x}), F_{k}^{p}(\mathbf{y})\right) \leq \frac{1}{m} \cdot \varphi(\rho(\mathbf{f}(\mathbf{x}), \mathbf{f}(\mathbf{y}))) \cdot \rho(\mathbf{f}(\mathbf{x}), \mathbf{f}(\mathbf{y}))
$$

are satisfied for all $k=1, \ldots, m$. Then the following statements hold true.

(i) There exists $\hat{\mathbf{x}} \in X^{m}$ of $\mathbf{F}$ such that $\mathbf{F}^{p}(\hat{\mathbf{x}})=\mathbf{f}(\hat{\mathbf{x}})$. If $p=1$, then $\hat{\mathbf{x}}$ is a coincidence point of $\mathbf{F}$ and $\mathbf{f}$.

(ii) If there exists $\hat{\mathbf{y}} \in X^{m}$ such that $\mathbf{F}^{p}(\hat{\mathbf{y}})=\mathbf{f}(\hat{\mathbf{y}})$ with $\hat{\mathbf{x}} \preccurlyeq_{I} \hat{\mathbf{y}}$ or $\hat{\mathbf{y}} \preccurlyeq_{I} \hat{\mathbf{x}}$, then $\mathbf{f}(\hat{\mathbf{x}})=\mathbf{f}(\hat{\mathbf{y}})$.

(iii) Suppose that $\hat{\mathbf{x}}$ is obtained from part (i). If $\hat{\mathbf{x}}$ and $\mathbf{F}(\hat{\mathbf{x}})$ are comparable with respect to ' $\preccurlyeq$ ', then $\mathbf{f}^{q}(\hat{\mathbf{x}})$ is a fixed point of $\mathbf{F}$ for any $q \in \mathbb{N}$

Moreover, each component $\hat{x}^{(k)}$ of $\hat{\mathbf{x}}$ is the limit of the sequence $\left\{f_{k}\left(\mathbf{x}_{n}\right)\right\}_{n \in \mathbb{N}}$ constructed in (3) for all $k=1, \ldots, m$.

Proof From the proof of Theorem 3.2, we can construct a sequence $\left\{\mathbf{x}_{n}\right\}_{n \in \mathbb{N}}$ in $X^{m}$ such that $f_{k}\left(\mathbf{x}_{n}\right) \rightarrow \hat{x}^{(k)}$ and $f_{k}\left(\mathbf{f}\left(\mathbf{x}_{n}\right)\right) \rightarrow f_{k}(\hat{\mathbf{x}})$ as $n \rightarrow \infty$ for all $k=1, \ldots, m$, where $\left\{\mathbf{f}\left(\mathbf{x}_{n}\right)\right\}_{n \in \mathbb{N}}$ is $\mathrm{a} \preccurlyeq{ }_{I}$-monotone sequence. From Remark 3.4, it follows that, for each $n \in \mathbb{N}, \mathbf{f}\left(\mathbf{x}_{n}\right) \preccurlyeq_{I} \hat{\mathbf{x}}$ or $\mathbf{f}\left(\mathbf{x}_{n}\right) \succcurlyeq_{I} \hat{\mathbf{x}}$. Since $f_{k}\left(\mathbf{f}\left(\mathbf{x}_{n}\right)\right) \rightarrow f_{k}(\hat{\mathbf{x}})$ as $n \rightarrow \infty$, given any $\epsilon>0$, there exists $n_{0} \in \mathbb{N}$ such that

$$
d\left(f_{k}\left(\mathbf{f}\left(\mathbf{x}_{n}\right)\right), f_{k}(\hat{\mathbf{x}})\right)<\frac{\epsilon}{2}
$$

for all $n \in \mathbb{N}$ with $n \geq n_{0}$ and for all $k=1, \ldots, m$. Since $\mathbf{f}$ is $\preccurlyeq_{I}$-monotone, it follows that $\mathbf{f}\left(\mathbf{f}\left(\mathbf{x}_{n}\right)\right) \preccurlyeq_{I} \mathbf{f}(\hat{\mathbf{x}})$ or $\mathbf{f}\left(\mathbf{f}\left(\mathbf{x}_{n}\right)\right) \succcurlyeq_{I} \mathbf{f}(\hat{\mathbf{x}})$. For each $n \geq n_{0}$, it follows that

$$
\begin{aligned}
d\left(F_{k}^{p}(\hat{\mathbf{x}}), F_{k}^{p}\left(\mathbf{f}\left(\mathbf{x}_{n}\right)\right)\right) & \leq \frac{1}{m} \cdot \varphi\left(\rho\left(\mathbf{f}(\hat{\mathbf{x}}), \mathbf{f}\left(\mathbf{f}\left(\mathbf{x}_{n}\right)\right)\right)\right) \cdot \rho\left(\mathbf{f}(\hat{\mathbf{x}}), \mathbf{f}\left(\mathbf{f}\left(\mathbf{x}_{n}\right)\right)\right) \quad(\text { by }(41)) \\
& <\frac{1}{m} \cdot \rho\left(\mathbf{f}(\hat{\mathbf{x}}), \mathbf{f}\left(\mathbf{f}\left(\mathbf{x}_{n}\right)\right)\right) \leq \frac{1}{m} \cdot \sum_{k=1}^{m} d\left(f_{k}(\hat{\mathbf{x}}), f_{k}\left(\mathbf{f}\left(\mathbf{x}_{n}\right)\right)\right) \quad(\text { by }(40)) \\
& <\frac{\epsilon}{2} \quad(\text { by }(42)) .
\end{aligned}
$$

Using the same argument in the proof of part (i) of Theorem 2.21, part (i) of this theorem follows immediately.

To prove part (ii), since $\mathbf{f}$ is $\preccurlyeq_{I}$-monotone, we immediately have $\mathbf{f}(\mathbf{y}) \preccurlyeq_{I} \mathbf{f}(\mathbf{x})$ or $\mathbf{f}(\mathbf{x}) \preccurlyeq_{I}$ $\mathbf{f}(\mathbf{y})$. If $f_{k}(\hat{\mathbf{x}}) \neq f_{k}(\hat{\mathbf{y}})$, i.e., $d\left(f_{k}(\hat{\mathbf{x}}), f_{k}(\hat{\mathbf{y}})\right) \neq 0$, then we obtain

$$
\begin{aligned}
0 & \neq \sum_{k=1}^{m} d\left(f_{k}(\hat{\mathbf{x}}), f_{k}(\hat{\mathbf{y}})\right)=\sum_{k=1}^{m} d\left(F_{k}^{p}(\hat{\mathbf{x}}), F_{k}^{p}(\hat{\mathbf{y}})\right) \\
& \leq \frac{1}{m} \cdot \sum_{k=1}^{m} \varphi(\rho(\mathbf{f}(\hat{\mathbf{x}}), \mathbf{f}(\hat{\mathbf{y}}))) \cdot \rho(\mathbf{f}(\hat{\mathbf{x}}), \mathbf{f}(\hat{\mathbf{y}})) \quad(\text { by }(41)) \\
& <\rho(\mathbf{f}(\hat{\mathbf{x}}), \mathbf{f}(\hat{\mathbf{y}})) \leq \sum_{k=1}^{m} d\left(f_{k}(\hat{\mathbf{x}}), f_{k}(\hat{\mathbf{y}})\right) \quad(\text { by }(40)) .
\end{aligned}
$$

This contradiction says that $f_{k}(\hat{\mathbf{x}})=f_{k}(\hat{\mathbf{y}})$ for all $k=1, \ldots, m$, i.e., $\mathbf{f}(\hat{\mathbf{x}})=\mathbf{f}(\hat{\mathbf{y}})$. Finally, part (iii) follows from the same argument in the proof of part (iii) of Theorem 2.21 immediately. This completes the proof. 
Remark 3.6 Suppose that inequalities (40) and (41) in Theorem 3.5 are satisfied for any $\mathbf{x}, \mathbf{y} \in X^{m}$. Then, from the proof of Theorem 3.5, we can see that parts (ii) and (iii) can be changed as follows.

(ii) $^{\prime} \quad$ If there exists $\hat{\mathbf{y}} \in X^{m}$ satisfying $\mathbf{F}^{p}(\hat{\mathbf{y}})=\mathbf{f}(\hat{\mathbf{y}})$, then $\mathbf{f}(\hat{\mathbf{x}})=\mathbf{f}(\hat{\mathbf{y}})$.

(iii)' Suppose that $\hat{\mathbf{x}}$ is obtained from part (i). Then $\mathbf{f}^{q}(\hat{\mathbf{x}})$ is a fixed point of $\mathbf{F}$ for any $q \in \mathbb{N}$.

Next, we shall study the $\preccurlyeq_{I}$-chain-uniqueness for the common fixed point, which is different from the chain-uniqueness in Definition 2.27.

Definition 3.7 Let $(X, \preceq)$ be a quasi-ordered set. Consider the functions $\mathbf{F}: X^{m} \rightarrow X^{m}$ and $\mathbf{f}: X^{m} \rightarrow X^{m}$ defined on the product set $X^{m}$ into itself. Given a disjoint pair $I$ and $J$ of $\{1, \ldots, m\}$, we recall that the chain $\mathfrak{C}\left(\preccurlyeq_{I}, \mathbf{x}\right)$ containing $\mathbf{x}$ is given by

$$
\mathfrak{C}\left(\preccurlyeq_{I}, \mathbf{x}\right)=\left\{\mathbf{y} \in X^{m}: \mathbf{y} \preccurlyeq_{I} \mathbf{x} \text { or } \mathbf{x} \preccurlyeq_{I} \mathbf{y}\right\}
$$

The common fixed point $\hat{\mathbf{x}} \in X^{m}$ of $\mathbf{F}$ and $\mathbf{f}$ is called $\preccurlyeq_{I}$-chain-unique if and only if, for any other common fixed point $\mathbf{x}$ of $\mathbf{F}$ and $\mathbf{f}$, if $\mathbf{x} \in \mathfrak{C}\left(\preccurlyeq{ }_{I}, \hat{\mathbf{x}}\right)$, then $\mathbf{x}=\hat{\mathbf{x}}$.

Theorem 3.8 Suppose that the quasi-ordered metric space $(X, d, \preceq)$ is monotonically complete and preserves the monotone convergence. Assume that the metrics $\mathfrak{d}$ and $d$ are compatible in the sense of preserving continuity. Consider the functions $\mathbf{F}:\left(X^{m}, \mathfrak{d}, \preccurlyeq_{I}\right) \rightarrow\left(X^{m}, \mathfrak{d}, \preccurlyeq_{I}\right)$ and $\mathbf{f}:\left(X^{m}, \mathfrak{d}, \preccurlyeq_{I}\right) \rightarrow\left(X^{m}, \mathfrak{d}, \preccurlyeq_{I}\right)$ satisfying $\mathbf{F}^{p}\left(X^{m}\right) \subseteq \mathbf{f}\left(X^{m}\right)$ for some $p \in \mathbb{N}$. Let $\mathbf{x}_{0}$ be a $\preccurlyeq_{I}$-monotone seed element in $X^{m}$. Assume that the functions $\mathbf{F}$ and $\mathbf{f}$ satisfy the following conditions:

- $\mathbf{F}$ and $\mathbf{f}$ are commutative;

- f $i s \preccurlyeq{ }_{I}$-monotone;

- $\mathbf{F}^{p}$ is continuous with respect to $\mathbf{f}$ on $X^{m}$;

- each $f_{k}$ is continuous on $X^{m}$ for $k=1, \ldots, m$.

Suppose that there exist a function $\rho: X^{m} \times X^{m} \rightarrow \mathbb{R}_{+}$and a function of contractive factor $\varphi:[0, \infty) \rightarrow[0,1)$ such that, for any $\mathbf{x}, \mathbf{y} \in X^{m}$ with $\mathbf{y} \preccurlyeq_{I} \mathbf{x}$ or $\mathbf{x} \preccurlyeq_{I} \mathbf{y}$, the following inequalities

$$
\rho(\mathbf{x}, \mathbf{y}) \leq \sum_{k=1}^{m} d\left(x^{(k)}, y^{(k)}\right)
$$

and

$$
d\left(F_{k}^{p}(\mathbf{x}), F_{k}^{p}(\mathbf{y})\right) \leq \frac{1}{m} \cdot \varphi(\rho(\mathbf{f}(\mathbf{x}), \mathbf{f}(\mathbf{y}))) \cdot \rho(\mathbf{f}(\mathbf{x}), \mathbf{f}(\mathbf{y}))
$$

are satisfied for all $k=1, \ldots, m$. Then the following statements hold true.

(i) $\mathbf{F}^{p}$ and $\mathbf{f}$ have $a \preccurlyeq_{I}$-chain-unique common fixed point $\hat{\mathbf{x}}$.

(ii) For $p \neq 1$, suppose that $\mathbf{F}(\hat{\mathbf{x}})$ and $\hat{\mathbf{x}}$ obtained in (i) are comparable with respect to ' $\preccurlyeq I$ '.

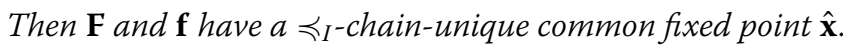

Moreover, each component $\hat{x}^{(k)}$ of $\hat{\mathbf{x}}$ is the limit of the sequence $\left\{f_{k}\left(\mathbf{x}_{n}\right)\right\}_{n \in \mathbb{N}}$ constructed in (3) for all $k=1, \ldots, m$. 
Proof To prove part (i), from Proposition 2.8 and part (i) of Theorem 3.5, we have $\mathbf{f}(\hat{\mathbf{x}})=$ $\mathbf{F}^{p}(\hat{\mathbf{x}})$. From Theorem 3.2, we also have $\mathbf{F}^{p}(\hat{\mathbf{x}})=\hat{\mathbf{x}}$. Therefore, we obtain

$$
\hat{\mathbf{x}}=\mathbf{f}(\hat{\mathbf{x}})=\mathbf{F}^{p}(\hat{\mathbf{x}})
$$

This shows that $\hat{\mathbf{x}}$ is a common fixed point of $\mathbf{F}^{p}$ and $\mathbf{f}$. For the $\preccurlyeq_{I}$-chain-uniqueness, let $\hat{\mathbf{y}}$ be another common fixed point of $\mathbf{F}^{p}$ and $\mathbf{f}$ such that $\hat{\mathbf{y}}$ and $\hat{\mathbf{x}}$ are comparable with respect to ' $\preccurlyeq I^{\prime}$, i.e., $\hat{\mathbf{y}}=\mathbf{f}(\hat{\mathbf{y}})=\mathbf{F}^{p}(\hat{\mathbf{y}})$. By part (ii) of Theorem 3.5 , we have $\mathbf{f}(\hat{\mathbf{x}})=\mathbf{f}(\hat{\mathbf{y}})$. Therefore, by the triangle inequality, we have

$$
d(\hat{\mathbf{x}}, \hat{\mathbf{y}}) \leq d(\hat{\mathbf{x}}, \mathbf{f}(\hat{\mathbf{x}}))+d(\mathbf{f}(\hat{\mathbf{x}}), \mathbf{f}(\hat{\mathbf{y}}))+d(\mathbf{f}(\hat{\mathbf{y}}), \hat{\mathbf{y}})=0
$$

which says that $\hat{\mathbf{x}}=\hat{\mathbf{y}}$. This proves part (i). Part (ii) can be obtained by applying part (iii) of Theorem 3.5 to a similar argument in the proof of Theorem 2.26. This completes the proof.

Theorem 3.9 Suppose that the quasi-ordered metric space $(X, d, \preceq)$ is monotonically complete and preserves the monotone convergence. Assume that the metrics $\mathfrak{d}$ and $d$ are compatible in the sense of preserving continuity. Consider the functions $\mathbf{F}:\left(X^{m}, \mathfrak{d}, \preccurlyeq_{I}\right) \rightarrow\left(X^{m}, \mathfrak{d}, \preccurlyeq_{I}\right)$ and $\mathbf{f}:\left(X^{m}, \mathfrak{d}, \preccurlyeq_{I}\right) \rightarrow\left(X^{m}, \mathfrak{d}, \preccurlyeq_{I}\right)$ satisfying $\mathbf{F}^{p}\left(X^{m}\right) \subseteq \mathbf{f}\left(X^{m}\right)$ for some $p \in \mathbb{N}$. Let $\mathbf{x}_{0}$ be a $\preccurlyeq_{I}$-monotone seed element in $X^{m}$. Assume that the functions $\mathbf{F}$ and $\mathbf{f}$ satisfy the following conditions:

- $\mathbf{F}$ and $\mathbf{f}$ are commutative;

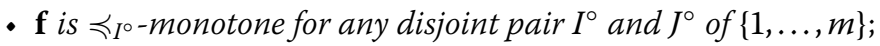

- $\mathbf{F}^{p}$ is continuous with respect to $\mathbf{f}$ on $X^{m}$;

- each $f_{k}$ is continuous on $X^{m}$ for $k=1, \ldots, m$.

Suppose that there exist a function $\rho: X^{m} \times X^{m} \rightarrow \mathbb{R}_{+}$and a function of contractive factor $\varphi:[0, \infty) \rightarrow[0,1)$ such that, for any $\mathbf{x}, \mathbf{y} \in X^{m}$ and any disjoint pair $I^{\circ}$ and $J^{\circ}$ of $\{1, \ldots, m\}$ with $\mathbf{y} \preccurlyeq I_{I^{\circ}} \mathbf{x}$ or $\mathbf{x} \preccurlyeq I_{I^{\circ}} \mathbf{y}$, the following inequalities

$$
\rho(\mathbf{x}, \mathbf{y}) \leq \sum_{k=1}^{m} d\left(x^{(k)}, y^{(k)}\right)
$$

and

$$
d\left(F_{k}^{p}(\mathbf{x}), F_{k}^{p}(\mathbf{y})\right) \leq \frac{1}{m} \cdot \varphi(\rho(\mathbf{f}(\mathbf{x}), \mathbf{f}(\mathbf{y}))) \cdot \rho(\mathbf{f}(\mathbf{x}), \mathbf{f}(\mathbf{y}))
$$

are satisfied for all $k=1, \ldots, m$. Then the following statements hold true.

(i) $\mathbf{F}^{p}$ and $\mathbf{f}$ have a chain-unique common fixed point $\hat{\mathbf{x}}$.

(ii) For $p \neq 1$, suppose that $\mathbf{F}(\hat{\mathbf{x}})$ and $\hat{\mathbf{x}}$ obtained in (i) are comparable with respect to the quasi-order ' $\preccurlyeq I^{\circ}$ ' for some disjoint pair $I^{\circ}$ and $J^{\circ}$ of $\{1, \ldots, m\}$. Then $\mathbf{F}$ and $\mathbf{f}$ have a chain-unique common fixed point $\hat{\mathbf{x}}$.

Moreover, each component $\hat{x}^{(k)}$ of $\hat{\mathbf{x}}$ is the limit of the sequence $\left\{f_{k}\left(\mathbf{x}_{n}\right)\right\}_{n \in \mathbb{N}}$ constructed in (3) for all $k=1, \ldots, m$.

Proof To prove part (i), from the proof of Theorem 3.8, we can show that $\hat{\mathbf{x}}$ is a common fixed point of $\mathbf{F}^{p}$ and $\mathbf{f}$. For the chain-uniqueness, let $\hat{\mathbf{y}}$ be another common fixed point of 
$\mathbf{F}^{p}$ and $\mathbf{f}$ with $\hat{\mathbf{y}} \preccurlyeq I^{\circ} \hat{\mathbf{x}}$ or $\hat{\mathbf{x}} \preccurlyeq I^{\circ} \hat{\mathbf{y}}$ for some disjoint pair $I^{\circ}$ and $J^{\circ}$ of $\{1, \ldots, m\}$, i.e., $\hat{\mathbf{y}}=\mathbf{f}(\hat{\mathbf{y}})=$ $\mathbf{F}^{p}(\hat{\mathbf{y}})$. Since $\mathbf{f}$ is $\preccurlyeq I^{\circ}$-monotone for any disjoint pair $I^{\circ}$ and $J^{\circ}$ of $\{1, \ldots, m\}$, we also have $\mathbf{f}(\hat{\mathbf{y}}) \preccurlyeq I_{I^{\circ}} \mathbf{f}(\hat{\mathbf{x}})$ or $\mathbf{f}(\hat{\mathbf{x}}) \preccurlyeq I^{\circ} \mathbf{f}(\hat{\mathbf{y}})$. If $f_{k}(\hat{\mathbf{x}}) \neq f_{k}(\hat{\mathbf{y}})$, i.e., $d\left(f_{k}(\hat{\mathbf{x}}), f_{k}(\hat{\mathbf{y}})\right) \neq 0$, then we obtain

$$
\begin{aligned}
0 & \neq \sum_{k=1}^{m} d\left(f_{k}(\hat{\mathbf{x}}), f_{k}(\hat{\mathbf{y}})\right)=\sum_{k=1}^{m} d\left(F_{k}^{p}(\hat{\mathbf{x}}), F_{k}^{p}(\hat{\mathbf{y}})\right) \\
& \leq \frac{1}{m} \cdot \sum_{k=1}^{m} \varphi(\rho(\mathbf{f}(\hat{\mathbf{x}}), \mathbf{f}(\hat{\mathbf{y}}))) \cdot \rho(\mathbf{f}(\hat{\mathbf{x}}), \mathbf{f}(\hat{\mathbf{y}})) \quad(\text { by }(47)) \\
& <\rho(\mathbf{f}(\hat{\mathbf{x}}), \mathbf{f}(\hat{\mathbf{y}})) \leq \sum_{k=1}^{m} d\left(f_{k}(\hat{\mathbf{x}}), f_{k}(\hat{\mathbf{y}})\right) \quad(\text { by }(46)) .
\end{aligned}
$$

This contradiction says that $f_{k}(\hat{\mathbf{x}})=f_{k}(\hat{\mathbf{y}})$ for all $k=1, \ldots, m$, i.e., $\mathbf{f}(\hat{\mathbf{x}})=\mathbf{f}(\hat{\mathbf{y}})$. Therefore, according to (45), we also have $\hat{\mathbf{x}}=\hat{\mathbf{y}}$. This proves part (i). Part (ii) can be similarly obtained by applying Theorem 2.22 to the argument in the proof of part (ii) of Theorem 2.26. This completes the proof.

Remark 3.10 We strongly assume that inequalities (43) and (44) in Theorem 3.8 and inequalities (46) and (47) in Theorem 3.9 are satisfied for any $\mathbf{x}, \mathbf{y} \in X^{m}$. Then, from Remark 3.6 and the proofs of Theorems 3.8 and 3.9, we can see that parts (i) and (ii) can be combined as $\mathbf{F}$ and $\mathbf{f}$ have a unique common fixed point $\hat{\mathbf{x}}$.

\section{Fixed points of functions having mixed monotone property in the product spaces}

We shall study the fixed points of functions having mixed monotone property in the product space. The concept of mixed monotone property for functions was adopted for presenting the coupled fixed point theorems.

Let $(X, \preceq)$ be a quasi-ordered set, and let $I$ and $J$ be the disjoint pair of $\{1,2, \ldots, m\}$. Consider the function $F: X^{m} \rightarrow X$.

- We say that $F$ is $\left(\preccurlyeq_{I}, \preceq\right)$-increasing if and only if $\mathbf{x} \preccurlyeq_{I} \mathbf{y}$ implies $F(\mathbf{x}) \preceq F(\mathbf{y})$.

- We say that $F$ is $\left(\preccurlyeq_{I}, \preceq\right)$-decreasing if and only if $\mathbf{x} \preccurlyeq_{I} \mathbf{y}$ implies $F(\mathbf{x}) \succeq F(\mathbf{y})$.

From (2), we see that $F$ is $(\preccurlyeq I, \preceq)$-decreasing if and only if it is $(\preccurlyeq J, \preceq)$-increasing, and $F$ is $\left(\preccurlyeq_{I}, \preceq\right)$-increasing if and only if it is $\left(\preccurlyeq_{J}, \preceq\right)$-decreasing.

Example 4.1 For $m=5$, we take the disjoint pair $I=\{1,2,4\}$ and $J=\{3,5\}$ of $\{1,2,3,4,5\}$. We say that the function $F: X^{5} \rightarrow X$ has the $I$-mixed monotone property if and only if the following conditions are satisfied:

- $F\left(x_{1}, x_{2}, x_{3}, x_{4}, x_{5}\right)$ is increasing in the corresponding variables $x_{1}, x_{2}, x_{4}$;

- $F\left(x_{1}, x_{2}, x_{3}, x_{4}, x_{5}\right)$ is decreasing in the corresponding variables $x_{3}, x_{5}$.

According to (1), it follows that $\mathbf{x} \preccurlyeq_{I} \mathbf{y}$ implies $F(\mathbf{x}) \preceq F(\mathbf{y})$, i.e., $F$ is $I$-increasing. Now, we assume that the function $F: X^{m} \rightarrow X$ has the $J$-mixed monotone property, i.e., the following conditions are satisfied:

- $F\left(x_{1}, x_{2}, x_{3}, x_{4}, x_{5}\right)$ is increasing in the corresponding variables $x_{3}, x_{5}$.

- $F\left(x_{1}, x_{2}, x_{3}, x_{4}, x_{5}\right)$ is decreasing in the corresponding variables $x_{1}, x_{2}, x_{4}$.

It follows that $\mathbf{x} \preccurlyeq_{J} \mathbf{y}$ implies $F(\mathbf{x}) \succeq F(\mathbf{y})$, i.e., $F$ is $J$-decreasing on $X^{m}$.

Based on Example 4.1, the general definition is given below. 
Definition 4.2 Let $(X, \preceq)$ be a quasi-ordered set, and let $I=\left\{r_{1}, \ldots, r_{u}\right\}$ and $J=\left\{s_{1}, \ldots, s_{v}\right\}$ be the disjoint pair of $\{1, \ldots, m\}$. We say that the function $F: X^{m} \rightarrow X$ has the $I$-mixed monotone property if and only if the following conditions are satisfied:

- $F$ is increasing in the $r_{1}$ th, $r_{2}$ th, $\ldots, r_{u}$ th positions, respectively; that is, if $x_{1}^{\left(r_{j}\right)} \preceq x_{2}^{\left(r_{j}\right)}$ for $j=1, \ldots, u$, then

$$
F\left(y^{(1)}, \ldots, y^{\left(r_{j}-1\right)}, x_{1}^{\left(r_{j}\right)}, y^{\left(r_{j}+1\right)}, \ldots, y^{(m)}\right) \preceq F\left(y^{(1)}, \ldots, y^{\left(r_{j}-1\right)}, x_{2}^{\left(r_{j}\right)}, y^{\left(r_{j}+1\right)}, \ldots, y^{(m)}\right)
$$

for all fixed $y^{(k)}$ with $k \neq r_{j}$ and $k=1, \ldots, m$;

- $F$ is decreasing in the $s_{1}$ th, $s_{2}$ th, .., $s_{v}$ th positions, respectively; that is, if $x_{1}^{\left(s_{i}\right)} \preceq x_{2}^{\left(s_{i}\right)}$ for $i=1, \ldots, v$, then

$$
F\left(y^{(1)}, \ldots, y^{\left(s_{i}-1\right)}, x_{2}^{\left(s_{i}\right)}, y^{\left(s_{i}+1\right)}, \ldots, y^{(m)}\right) \preceq F\left(y^{(1)}, \ldots, y^{\left(s_{i}-1\right)}, x_{1}^{\left(s_{i}\right)}, y^{\left(s_{i}+1\right)}, \ldots, y^{(m)}\right)
$$

for all fixed $y^{(k)}$ with $k \neq s_{i}$ and $k=1, \ldots, m$.

It can be realized that the function $F: X^{m} \rightarrow X$ has the $J$-mixed monotone property if and only if the following conditions are satisfied:

- $F$ is increasing in the $s_{1}$ th, $s_{2}$ th, .., $s_{\nu}$ th positions, respectively; that is, if $y_{1}^{\left(s_{i}\right)} \preceq y_{2}^{\left(s_{i}\right)}$, then

$$
F\left(x^{(1)}, \ldots, x^{\left(s_{i}-1\right)}, y_{1}^{\left(s_{i}\right)}, x^{\left(s_{i}+1\right)}, \ldots, x^{(m)}\right) \preceq F\left(x^{(1)}, \ldots, x^{\left(s_{i}-1\right)}, y_{2}^{\left(s_{i}\right)}, x^{\left(s_{i}+1\right)}, \ldots, x^{(m)}\right)
$$

for each $i=1, \ldots, v$;

- $F$ is decreasing in the $r_{1}$ th,$r_{2}$ th, $\ldots, r_{u}$ th positions, respectively; that is, if $y_{1}^{\left(r_{j}\right)} \preceq y_{2}^{\left(r_{j}\right)}$, then

$$
F\left(x^{(1)}, \ldots, x^{\left(r_{j}-1\right)}, y_{2}^{\left(r_{j}\right)}, x^{\left(r_{j}+1\right)}, \ldots, x^{(m)}\right) \preceq F\left(x^{(1)}, \ldots, x^{\left(r_{j}-1\right)}, y_{1}^{\left(r_{j}\right)}, x^{\left(r_{j}+1\right)}, \ldots, x^{(m)}\right)
$$

for each $j=1, \ldots, u$.

Remark 4.3 According to (1), if the function $F: X^{m} \rightarrow X$ has the $I$-mixed monotone property, then $F$ is $\left(\preccurlyeq_{I}, \preceq\right)$-increasing. Also, if the function $F: X^{m} \rightarrow X$ has the $J$-mixed monotone property, then $F$ is $(\preccurlyeq J, \preceq)$-increasing $\left(i . e .,(\preccurlyeq I, \preceq)\right.$-decreasing) on $X^{m}$.

Now, considering the function $\mathbf{F}: X^{m} \rightarrow X^{m}$, we are going to define many monotonic concepts of $\mathbf{F}$ on $X^{m}$ as follows.

Definition 4.4 Let $(X, \preceq)$ be a quasi-ordered set, and let $I$ and $J$ be the disjoint pair of $\{1,2, \ldots, m\}$. Consider the quasi-ordered set $\left(X^{m}, \preccurlyeq_{I}\right)$ and the function $\mathbf{F}:\left(X^{m}, \preccurlyeq_{I}\right) \rightarrow$ $\left(X^{m}, \preccurlyeq_{I}\right)$.

- We say that $\mathbf{F}$ is $\left(\preccurlyeq_{I}, \preccurlyeq_{I}\right)$-increasing if and only if $\mathbf{x} \preccurlyeq{ }_{I} \mathbf{y}$ implies $\mathbf{F}(\mathbf{x}) \preccurlyeq_{I} \mathbf{F}(\mathbf{y})$.

- We say that $\mathbf{F}$ is $\left(\preccurlyeq_{I}, \preccurlyeq_{J}\right)$-increasing if and only if $\mathbf{x} \preccurlyeq_{I} \mathbf{y}$ implies $\mathbf{F}(\mathbf{x}) \preccurlyeq_{J} \mathbf{F}(\mathbf{y})$.

- We say that $\mathbf{F}$ is $\left(\preccurlyeq_{J}, \preccurlyeq_{I}\right)$-increasing if and only if $\mathbf{x} \preccurlyeq J \mathbf{y}$ implies $\mathbf{F}(\mathbf{x}) \preccurlyeq_{I} \mathbf{F}(\mathbf{y})$.

- We say that $\mathbf{F}$ is $\left(\preccurlyeq_{J}, \preccurlyeq_{J}\right)$-increasing if and only if $\mathbf{x} \preccurlyeq_{J} \mathbf{y}$ implies $\mathbf{F}(\mathbf{x}) \preccurlyeq_{J} \mathbf{F}(\mathbf{y})$.

- We say that $\mathbf{F}$ is $\left(\preccurlyeq_{I}, \preccurlyeq_{I}\right)$-decreasing if and only if $\mathbf{x} \preccurlyeq_{I} \mathbf{y}$ implies $\mathbf{F}(\mathbf{x}) \succcurlyeq_{I} \mathbf{F}(\mathbf{y})$.

- We say that $\mathbf{F}$ is $\left(\preccurlyeq_{I}, \preccurlyeq_{J}\right)$-decreasing if and only if $\mathbf{x} \preccurlyeq_{I} \mathbf{y}$ implies $\mathbf{F}(\mathbf{x}) \succcurlyeq_{J} \mathbf{F}(\mathbf{y})$. 
- We say that $\mathbf{F}$ is $\left(\preccurlyeq_{J}, \preccurlyeq_{I}\right)$-decreasing if and only if $\mathbf{x} \preccurlyeq_{J} \mathbf{y}$ implies $\mathbf{F}(\mathbf{x}) \succcurlyeq_{I} \mathbf{F}(\mathbf{y})$.

- We say that $\mathbf{F}$ is $\left(\preccurlyeq_{J}, \preccurlyeq_{J}\right)$-decreasing if and only if $\mathbf{x} \preccurlyeq_{J} \mathbf{y}$ implies $\mathbf{F}(\mathbf{x}) \succcurlyeq_{J} \mathbf{F}(\mathbf{y})$.

Remark 4.5 From (2), we see that it suffices to consider the increasing cases. On the other hand, we also see that $\mathbf{F}$ is $\left(\preccurlyeq_{I}, \preccurlyeq_{I}\right)$-increasing if and only if it is $\left(\preccurlyeq_{J}, \preccurlyeq_{J}\right)$-increasing, and $\mathbf{F}$ is $\left(\preccurlyeq_{I}, \preccurlyeq_{J}\right)$-increasing if and only if it is $\left(\preccurlyeq_{J}, \preccurlyeq_{I}\right)$-increasing. Therefore, the cases in Definition 4.4 can be reduced to only consider the $\left(\preccurlyeq_{I}, \preccurlyeq_{I}\right)$-increasing and $\left(\preccurlyeq_{I}, \preccurlyeq_{J}\right)$-increasing cases. Since the $\left(\preccurlyeq_{I}, \preccurlyeq_{J}\right)$-increasing case is equivalent to the $\left(\preccurlyeq_{I}, \preccurlyeq_{I}\right)$-decreasing case, it follows that the cases in Definition 4.4 can be reduced to only consider the $\left(\preccurlyeq_{I}, \preccurlyeq_{I}\right)$ increasing and $\left(\preccurlyeq_{I}, \preccurlyeq_{I}\right)$-decreasing cases.

Definition 4.6 Let $(X, \preceq)$ be a quasi-ordered set, and let $I=\left\{r_{1}, \ldots, r_{u}\right\}$ and $J=\left\{v_{1}, \ldots, v_{s}\right\}$ be the disjoint pair of $\{1,2, \ldots, m\}$. We say that the function $\mathbf{F}: X^{m} \rightarrow X^{m}$ has the $I$-mixed monotone property on $X^{m}$ if and only if its $k$ th component function $F_{k}$ satisfies the following conditions:

- $F_{k}$ has the $I$-mixed monotone property for $k \in I$; in other words, $F_{k}$ is increasing in the $r_{1}$ th, $r_{2}$ th $, \ldots, r_{u}$ th positions and is decreasing in the $s_{1}$ th, $s_{2}$ th, $\ldots, s_{\nu}$ th positions, respectively;

- $F_{k}$ has the $J$-mixed monotone property for $k \in J$; in other words, $F_{k}$ is decreasing in the $r_{1}$ th, $r_{2}$ th, $\ldots, r_{u}$ th positions and is increasing in the $s_{1}$ th, $s_{2}$ th, $\ldots, s_{v}$ th positions, respectively.

The meaning of the $J$-mixed monotone property for $\mathbf{F}$ can be similarly realized.

Remark 4.7 According to (1), if the function $\mathbf{F}: X^{m} \rightarrow X^{m}$ has the $I$-mixed monotone property, then $\mathbf{F}$ is $\left(\preccurlyeq_{I}, \preccurlyeq_{I}\right)$-increasing. Also, if the function $\mathbf{F}: X^{m} \rightarrow X^{m}$ has the $J$-mixed monotone property, then $\mathbf{F}$ is $\left(\preccurlyeq_{I}, \preccurlyeq_{J}\right)$-increasing, i.e., $\left(\preccurlyeq_{I}, \preccurlyeq_{I}\right)$-decreasing.

Example 4.8 Continued from Example 4.1, the function $\mathbf{F}: X^{5} \rightarrow X^{5}$ has the $I$-mixed monotone property if and only if the component functions $F_{k}$ of $\mathbf{F}$ for $k=1, \ldots, 5$ satisfy the following conditions:

- the component functions $F_{k}$ have the $I$-mixed monotone property for $k \in I$;

- the component functions $F_{k}$ have the $J$-mixed monotone property for $k \in J$.

Example 4.9 Continued from Example 4.8, given the initial element $\mathbf{x}_{0} \in X^{5}$, we consider the iteration $\mathbf{x}_{n}=\mathbf{F}\left(\mathbf{x}_{n-1}\right)$. Then, according to (4), we can define two quasi-ordered sets $\left(X^{m}, \preccurlyeq_{I}\right)$ and $\left(X^{m}, \preccurlyeq_{J}\right)$ as follows.

(i) Assume that $\mathbf{x}_{0} \preccurlyeq_{I} \mathbf{x}_{1}$, i.e., $x_{0}^{(k)} \preceq x_{1}^{(k)}$ for $k \in I$ and $x_{1}^{(k)} \preceq x_{0}^{(k)}$ for $k \in J$.

- For $k \in I$, we are going to claim $x_{1}^{(k)} \preceq x_{2}^{(k)}$. This can be realized by simply checking the case of $k=2$. By definition, we see that $F_{2}\left(x_{1}, x_{2}, x_{3}, x_{4}, x_{5}\right)$ is increasing in the corresponding variables $x_{1}, x_{2}, x_{4}$, and is decreasing in the corresponding variables $x_{3}, x_{5}$. Therefore, we obtain

$$
\begin{aligned}
x_{1}^{(2)} & =F_{2}\left(x_{0}^{(1)}, x_{0}^{(2)}, x_{0}^{(3)}, x_{0}^{(4)}, x_{0}^{(5)}\right) \preceq F_{2}\left(x_{1}^{(1)}, x_{1}^{(2)}, x_{0}^{(3)}, x_{1}^{(4)}, x_{0}^{(5)}\right) \\
& \preceq F_{2}\left(x_{1}^{(1)}, x_{1}^{(2)}, x_{1}^{(3)}, x_{1}^{(4)}, x_{1}^{(5)}\right)=x_{2}^{(2)}
\end{aligned}
$$

- For $k \in J$, we are going to claim $x_{1}^{(k)} \succeq x_{2}^{(k)}$. This can be realized by simply checking the case of $k=5$. By definition, we see that $F_{5}\left(x_{1}, x_{2}, x_{3}, x_{4}, x_{5}\right)$ is 
increasing in the corresponding variables $x_{3}, x_{5}$, and is decreasing in the corresponding variables $x_{1}, x_{2}, x_{4}$. Therefore, we obtain

$$
\begin{aligned}
x_{1}^{(5)} & =F_{5}\left(x_{0}^{(1)}, x_{0}^{(2)}, x_{0}^{(3)}, x_{0}^{(4)}, x_{0}^{(5)}\right) \succeq F_{5}\left(x_{0}^{(1)}, x_{0}^{(2)}, x_{1}^{(3)}, x_{0}^{(4)}, x_{1}^{(5)}\right) \\
& \succeq F_{5}\left(x_{1}^{(1)}, x_{1}^{(2)}, x_{1}^{(3)}, x_{1}^{(4)}, x_{1}^{(5)}\right)=x_{2}^{(5)}
\end{aligned}
$$

By induction, we can show that the sequences $\left\{x_{n}^{(k)}\right\}$ are $\preceq$-increasing for $k \in I$, and are $\preceq$-decreasing for $k \in J$. Therefore, according to (4), we can induce a quasi-ordered set $\left(X^{m}, \preccurlyeq_{I}\right)$.

(ii) Assume that $\mathbf{x}_{1} \preccurlyeq_{I} \mathbf{x}_{0}$, i.e., $x_{0}^{(k)} \succeq x_{1}^{(k)}$ for $k \in I$ and $x_{1}^{(k)} \succeq x_{0}^{(k)}$ for $k \in J$. Equivalently, we have $\mathbf{x}_{0} \preccurlyeq_{J} \mathbf{x}_{1}$ by (2). We can similarly show that the sequences $\left\{x_{n}^{(k)}\right\}$ are $\preceq$-decreasing for $k \in I$, and are $\preceq$-increasing for $k \in J$. Therefore, according to (4), we can induce a quasi-ordered set $\left(X^{m}, \preccurlyeq J\right)$.

By referring to Example 4.9, we have the following general result.

Lemma 4.10 Let $(X, \preceq)$ be a quasi-ordered set, and let $I$ and $J$ be the disjoint pair of $\{1,2, \ldots, m\}$. Assume that the function $\mathbf{F}:\left(X^{m}, \preccurlyeq_{I}\right) \rightarrow\left(X^{m}, \preccurlyeq_{I}\right)$ has the I-mixed monotone property on $X^{m}$. Given the initial element $\mathbf{x}_{0} \in X^{m}$, we define the sequence $\left\{\mathbf{x}_{n}\right\}_{n \in \mathbb{N}}$ by $\mathbf{x}_{n}=\mathbf{F}\left(\mathbf{x}_{n-1}\right)$. Then the following statements hold true.

(i) Suppose that $\mathbf{x}_{0} \preccurlyeq_{I} \mathbf{x}_{1}$. Then the sequences $\left\{x_{n}^{(k)}\right\}_{n \in \mathbb{N}}$ are $\preceq$-increasing for each $k \in I$ and are $\preceq$-decreasing for each $k \in J$. In other words, $\left\{\mathbf{x}_{n}\right\}_{n \in \mathbb{N}}$ is a $\preccurlyeq_{I}$-increasing sequence.

(ii) Suppose that $\mathbf{x}_{0} \succcurlyeq_{I} \mathbf{x}_{1}$. Then the sequences $\left\{x_{n}^{(k)}\right\}_{n \in \mathbb{N}}$ are $\preceq$-decreasing for each $k \in I$ and are $\preceq$-increasing for each $k \in J$. In other words, $\left\{\mathbf{x}_{n}\right\}_{n \in \mathbb{N}}$ is $a \preccurlyeq{ }_{I}$-decreasing sequence.

Moreover, the initial element $\mathbf{x}_{0}$ is $a \preccurlyeq_{I}$-monotone seed element in $X^{m}$.

Proof To prove part (i), we have the following cases:

- If $k \in I$, then $F_{k}$ has the $I$-mixed monotone property. Therefore, we have

$$
x_{1}^{(k)}=F_{k}\left(x_{0}^{(1)}, \ldots, x_{0}^{(k)}, \ldots, x_{0}^{(m)}\right) \preceq \cdots \preceq F_{k}\left(x_{1}^{(1)}, \ldots, x_{1}^{(k)}, \ldots, x_{1}^{(m)}\right)=x_{2}^{(k)}
$$

by checking one component at each time.

- If $k \in J$, then $F_{k}$ has the $J$-mixed monotone property. Therefore, we have

$$
x_{1}^{(k)}=F_{k}\left(x_{0}^{(1)}, \ldots, x_{0}^{(k)}, \ldots, x_{0}^{(m)}\right) \succeq \cdots \succeq F_{k}\left(x_{1}^{(1)}, \ldots, x_{1}^{(k)}, \ldots, x_{1}^{(m)}\right)=x_{2}^{(k)}
$$

by checking one component at each time.

Therefore, by induction, part (i) follows immediately. A similar argument can also apply to part (ii), and the proof is complete.

Theorem 4.11 Suppose that the quasi-ordered metric space $(X, d, \preceq)$ is monotonically complete, and that the metrics $\mathfrak{d}$ and $d$ are compatible in the sense of preserving continuity. Given a disjoint pair I and J of $\{1,2, \ldots, m\}$, assume that the function $\mathbf{F}:\left(X^{m}, \mathfrak{d}, \preccurlyeq_{I}\right) \rightarrow$ $\left(X^{m}, \mathfrak{d}, \preccurlyeq_{I}\right)$ is continuous on $X^{m}$ and has the I-mixed monotone property, and that there 
exist a function $\rho: X^{m} \times X^{m} \rightarrow \mathbb{R}_{+}$and a function of contractive factor $\varphi:[0, \infty) \rightarrow[0,1)$ such that, for any $\mathbf{x}, \mathbf{y} \in X^{m}$ with $\mathbf{y} \preccurlyeq_{I} \mathbf{x}$ or $\mathbf{x} \preccurlyeq_{I} \mathbf{y}$, the following inequalities are satisfied:

$$
\rho(\mathbf{x}, \mathbf{y}) \leq \sum_{k=1}^{m} d\left(x^{(k)}, y^{(k)}\right)
$$

and

$$
d\left(F_{k}^{p}(\mathbf{x}), F_{k}^{p}(\mathbf{y})\right) \leq \frac{1}{m} \cdot \varphi(\rho(\mathbf{f}(\mathbf{x}), \mathbf{f}(\mathbf{y}))) \cdot \rho(\mathbf{f}(\mathbf{x}), \mathbf{f}(\mathbf{y}))
$$

for all $k=1, \ldots, m$ and for some $p \in \mathbb{N}$. If there exists $\mathbf{x}_{0} \in X^{m}$ such that $\mathbf{x}_{0} \preccurlyeq{ }_{I} \mathbf{F}^{p}\left(\mathbf{x}_{0}\right)$ or $\mathbf{x}_{0} \succcurlyeq_{I} \mathbf{F}^{p}\left(\mathbf{x}_{0}\right)$, then the function $\mathbf{F}^{p}$ has a fixed point $\hat{\mathbf{x}} \in X^{m}$, where each component $\hat{x}^{(k)}$ of $\hat{\mathbf{x}}$ is the limit of the sequence $\left\{x_{n}^{(k)}\right\}_{n \in \mathbb{N}}$ constructed below

$$
\mathbf{x}_{n}=\mathbf{F}^{p}\left(\mathbf{x}_{n-1}\right)
$$

for all $k=1, \ldots, m$.

Proof According to (48), we have $\mathbf{x}_{0} \preccurlyeq_{I} \mathbf{x}_{1}$ or $\mathbf{x}_{0} \succcurlyeq_{I} \mathbf{x}_{1}$. From Lemma 4.10, the initial element $\mathbf{x}_{0}$ is a $\preccurlyeq_{I}$-monotone seed element in $X^{m}$. Therefore, the results follow immediately from Theorem 3.2 by taking $\mathbf{f}$ as the identity function. This completes the proof.

Next, we can consider the chain-uniqueness and drop the assumption of continuity of $\mathbf{F}$ by assuming that $(X, d, \preceq)$ preserves the monotone convergence.

Theorem 4.12 Suppose that the quasi-ordered metric space $(X, d, \preceq)$ is monotonically complete and preserves the monotone convergence, and that the metrics $\mathfrak{d}$ and $d$ are compatible in the sense of preserving convergence. Given a disjoint pair I and $J$ of $\{1,2, \ldots, m\}$, assume that the function $\mathbf{F}:\left(X^{m}, \mathfrak{d}, \preccurlyeq_{I}\right) \rightarrow\left(X^{m}, \mathfrak{d}, \preccurlyeq_{I}\right)$ has the I-mixed monotone property, and that there exist a function $\rho: X^{m} \times X^{m} \rightarrow \mathbb{R}_{+}$and a function of contractive factor $\varphi:[0, \infty) \rightarrow[0,1)$ such that, for any $\mathbf{x}, \mathbf{y} \in X^{m}$ with $\mathbf{y} \preccurlyeq_{I} \mathbf{x}$ or $\mathbf{x} \preccurlyeq_{I} \mathbf{y}$, the following inequalities are satisfied:

$$
\rho(\mathbf{x}, \mathbf{y}) \leq \sum_{k=1}^{m} d\left(x^{(k)}, y^{(k)}\right)
$$

and

$$
d\left(F_{k}^{p}(\mathbf{x}), F_{k}^{p}(\mathbf{y})\right) \leq \frac{1}{m} \cdot \varphi(\rho(\mathbf{f}(\mathbf{x}), \mathbf{f}(\mathbf{y}))) \cdot \rho(\mathbf{f}(\mathbf{x}), \mathbf{f}(\mathbf{y}))
$$

for all $k=1, \ldots, m$ and for some $p \in \mathbb{N}$. If there exists $\mathbf{x}_{0} \in X^{m}$ such that $\mathbf{x}_{0} \preccurlyeq{ }_{I} \mathbf{F}^{p}\left(\mathbf{x}_{0}\right)$ or $\mathbf{x}_{0} \succcurlyeq_{I} \mathbf{F}^{p}\left(\mathbf{x}_{0}\right)$, then the function $\mathbf{F}^{p}$ has a $\preccurlyeq_{I}$-chain-unique fixed point $\hat{\mathbf{x}} \in X^{m}$, where each component $\hat{x}^{(k)}$ of $\hat{\mathbf{x}}$ is the limit of the sequence $\left\{x_{n}^{(k)}\right\}_{n \in \mathbb{N}}$ constructed from (48) for all $k=$ $1, \ldots, m$.

Proof According to (48), we have $\mathbf{x}_{0} \preccurlyeq_{I} \mathbf{x}_{1}$ or $\mathbf{x}_{0} \succcurlyeq_{I} \mathbf{x}_{1}$. From Lemma 4.10, the initial element $\mathbf{x}_{0}$ is a $\preccurlyeq_{I}$-monotone seed element in $X^{m}$. Therefore, the results follow immediately from Theorem 3.5 by taking $\mathbf{f}$ as the identity function. This completes the proof. 
The following fixed point theorem considers the different monotone conditions for $\mathbf{F}$.

Theorem 4.13 Suppose that the quasi-ordered metric space $(X, d, \preceq)$ is monotonically complete, and that the metrics $\mathfrak{d}$ and $d$ are compatible in the sense of preserving continuity. Given a disjoint pair $I$ and $J$ of $\{1,2, \ldots, m\}$, assume that the function $\mathbf{F}:\left(X^{m}, \mathfrak{d}, \preccurlyeq_{I}\right) \rightarrow$ $\left(X^{m}, \mathfrak{d}, \preccurlyeq_{I}\right)$ is continuous on $X^{m}$ and satisfies any one of the following conditions:

(a) $\mathbf{F}$ is $\left(\preccurlyeq_{I}, \preccurlyeq_{I}\right)$-increasing;

(b) $p$ is an even integer and $\mathbf{F}$ is $\left(\preccurlyeq_{I}, \preccurlyeq_{I}\right)$-decreasing.

Assume that there exist a function $\rho: X^{m} \times X^{m} \rightarrow \mathbb{R}_{+}$and a function of contractive factor $\varphi$ : $[0, \infty) \rightarrow[0,1)$ such that, for any $\mathbf{x}, \mathbf{y} \in X^{m}$ with $\mathbf{y} \preccurlyeq_{I} \mathbf{x}$ or $\mathbf{x} \preccurlyeq_{I} \mathbf{y}$, the following inequalities

$$
\rho(\mathbf{x}, \mathbf{y}) \leq \sum_{k=1}^{m} d\left(x^{(k)}, y^{(k)}\right)
$$

and

$$
d\left(F_{k}^{p}(\mathbf{x}), F_{k}^{p}(\mathbf{y})\right) \leq \frac{1}{m} \cdot \varphi(\rho(\mathbf{f}(\mathbf{x}), \mathbf{f}(\mathbf{y}))) \cdot \rho(\mathbf{f}(\mathbf{x}), \mathbf{f}(\mathbf{y}))
$$

are satisfied for all $k=1, \ldots, m$ and for some $p \in \mathbb{N}$. If there exists $\mathbf{x}_{0} \in X^{m}$ such that $\mathbf{x}_{0} \preccurlyeq_{I}$ $\mathbf{F}^{p}\left(\mathbf{x}_{0}\right)$ or $\mathbf{x}_{0} \succcurlyeq_{I} \mathbf{F}^{p}\left(\mathbf{x}_{0}\right)$, then the function $\mathbf{F}^{p}$ has a fixed point $\hat{\mathbf{x}} \in X^{m}$, where each component $\hat{x}^{(k)}$ of $\hat{\mathbf{x}}$ is the limit of the sequence $\left\{x_{n}^{(k)}\right\}_{n \in \mathbb{N}}$ constructed from (48) for all $k=1, \ldots, m$.

Proof We consider the following cases.

- If $\mathbf{F}$ is $\left(\preccurlyeq_{I}, \preccurlyeq_{I}\right)$-increasing, then it follows that $\mathbf{F}^{p}$ is $\left(\preccurlyeq_{I}, \preccurlyeq_{I}\right)$-increasing.

- If $\mathbf{F}$ is $\left(\preccurlyeq_{I}, \preccurlyeq_{I}\right)$-decreasing and $p$ is an even integer, then $\mathbf{F}^{p}$ is also $\left(\preccurlyeq_{I}, \preccurlyeq_{I}\right)$-increasing. According to (48), we have $\mathbf{x}_{0} \preccurlyeq_{I} \mathbf{x}_{1}$ or $\mathbf{x}_{0} \succcurlyeq_{I} \mathbf{x}_{1}$. Since $\mathbf{x}_{1}=\mathbf{F}^{p}\left(\mathbf{x}_{0}\right)$ and $\mathbf{x}_{2}=\mathbf{F}^{p}\left(\mathbf{x}_{1}\right)$, it follows that $\mathbf{x}_{0} \preccurlyeq_{I} \mathbf{x}_{1}$ implies $\mathbf{x}_{1} \preccurlyeq_{I} \mathbf{x}_{2}$, and $\mathbf{x}_{0} \succcurlyeq_{I} \mathbf{x}_{1}$ implies $\mathbf{x}_{1} \succcurlyeq_{I} \mathbf{x}_{2}$. Therefore, if $\mathbf{x}_{0} \preccurlyeq_{I} \mathbf{x}_{1}$, then we can generate a $\preccurlyeq_{I}$-increasing sequence $\left\{\mathbf{x}_{n}\right\}_{n \in \mathbb{N}}$, and if $\mathbf{x}_{0} \succcurlyeq_{I} \mathbf{x}_{1}$, then we can generate a $\preccurlyeq_{I}$-decreasing sequence $\left\{\mathbf{x}_{n}\right\}_{n \in \mathbb{N}}$, which also says that the initial element $\mathbf{x}_{0}$ is a $\preccurlyeq_{I}$-monotone seed element in $X^{m}$. Therefore, the results follow immediately from Theorem 3.2 by taking $\mathbf{f}$ as the identity function. This completes the proof.

Next, we can consider the chain-uniqueness and drop the assumption of continuity of $\mathbf{F}$ by assuming that $(X, d, \preceq)$ preserves the monotone convergence.

Theorem 4.14 Suppose that the quasi-ordered metric space $(X, d, \preceq)$ is monotonically complete and preserves the monotone convergence, and that the metrics $\mathfrak{d}$ and $d$ are compatible in the sense of preserving convergence. Given a disjoint pair I and $J$ of $\{1,2, \ldots, m\}$, assume that the function $\mathbf{F}:\left(X^{m}, \mathfrak{d}, \preccurlyeq_{I}\right) \rightarrow\left(X^{m}, \mathfrak{d}, \preccurlyeq_{I}\right)$ satisfies any one of the following conditions:

(a) $\mathbf{F}$ is $\left(\preccurlyeq_{I}, \preccurlyeq_{I}\right)$-increasing;

(b) $p$ is an even integer and $\mathbf{F}$ is $\left(\preccurlyeq_{I}, \preccurlyeq_{I}\right)$-decreasing.

Assume that there exist a function $\rho: X^{m} \times X^{m} \rightarrow \mathbb{R}_{+}$and a function of contractive factor $\varphi$ : $[0, \infty) \rightarrow[0,1)$ such that, for any $\mathbf{x}, \mathbf{y} \in X^{m}$ with $\mathbf{y} \preccurlyeq_{I} \mathbf{x}$ or $\mathbf{x} \preccurlyeq_{I} \mathbf{y}$, the following inequalities

$$
\rho(\mathbf{x}, \mathbf{y}) \leq \sum_{k=1}^{m} d\left(x^{(k)}, y^{(k)}\right)
$$


and

$$
d\left(F_{k}^{p}(\mathbf{x}), F_{k}^{p}(\mathbf{y})\right) \leq \frac{1}{m} \cdot \varphi(\rho(\mathbf{f}(\mathbf{x}), \mathbf{f}(\mathbf{y}))) \cdot \rho(\mathbf{f}(\mathbf{x}), \mathbf{f}(\mathbf{y}))
$$

are satisfied for all $k=1, \ldots, m$ and for some $p \in \mathbb{N}$. If there exists $\mathbf{x}_{0} \in X^{m}$ such that $\mathbf{x}_{0} \preccurlyeq_{I}$ $\mathbf{F}^{p}\left(\mathbf{x}_{0}\right)$ or $\mathbf{x}_{0} \succcurlyeq_{I} \mathbf{F}^{p}\left(\mathbf{x}_{0}\right)$, then the function $\mathbf{F}^{p}$ has a $\preccurlyeq_{I}$-chain-unique fixed point $\hat{\mathbf{x}} \in X^{m}$, where each component $\hat{x}^{(k)}$ of $\hat{\mathbf{x}}$ is the limit of the sequence $\left\{x_{n}^{(k)}\right\}_{n \in \mathbb{N}}$ constructed from (48) for all $k=1, \ldots, m$.

Proof From the proof of Theorem 4.13, we see that the initial element $\mathbf{x}_{0}$ is a $\preccurlyeq_{I}$-monotone seed element in $X^{m}$. Therefore, the results follow immediately from Theorem 3.5 by taking f as the identity function. This completes the proof.

The following fixed point theorem considers the odd integer $p \in \mathbb{N}$ and the different monotone conditions for $\mathbf{F}$.

Theorem 4.15 Suppose that the quasi-ordered metric space $(X, d, \preceq)$ is mixed-monotonically complete, and that the metrics $\mathfrak{d}$ and $d$ are compatible in the sense of preserving continuity. Given a disjoint pair $I$ and $J$ of $\{1,2, \ldots, m\}$, assume that the function $\mathbf{F}:\left(X^{m}, \mathfrak{d}, \preccurlyeq_{I}\right) \rightarrow\left(X^{m}, \mathfrak{d}, \preccurlyeq_{I}\right)$ is continuous on $X^{m}$ and $\left(\preccurlyeq_{I}, \preccurlyeq_{I}\right)$-decreasing, and that there exist a function $\rho: X^{m} \times X^{m} \rightarrow \mathbb{R}_{+}$and a function of contractive factor $\varphi:[0, \infty) \rightarrow[0,1)$ such that, for any $\mathbf{x}, \mathbf{y} \in X^{m}$ with $\mathbf{y} \preccurlyeq_{I} \mathbf{x}$ or $\mathbf{x} \preccurlyeq_{I} \mathbf{y}$, the following inequalities are satisfied:

$$
\rho(\mathbf{x}, \mathbf{y}) \leq \sum_{k=1}^{m} d\left(x^{(k)}, y^{(k)}\right)
$$

and

$$
d\left(F_{k}^{p}(\mathbf{x}), F_{k}^{p}(\mathbf{y})\right) \leq \frac{1}{m} \cdot \varphi(\rho(\mathbf{f}(\mathbf{x}), \mathbf{f}(\mathbf{y}))) \cdot \rho(\mathbf{f}(\mathbf{x}), \mathbf{f}(\mathbf{y}))
$$

for all $k=1, \ldots, m$ and for some odd integer $p \in \mathbb{N}$. If there exists $\mathbf{x}_{0} \in X^{m}$ such that $\mathbf{x}_{0} \preccurlyeq I$ $\mathbf{F}^{p}\left(\mathbf{x}_{0}\right)$ or $\mathbf{x}_{0} \succcurlyeq_{I} \mathbf{F}^{p}\left(\mathbf{x}_{0}\right)$, then the function $\mathbf{F}^{p}$ has a fixed point $\hat{\mathbf{x}} \in X^{m}$, where each component $\hat{x}^{(k)}$ of $\hat{\mathbf{x}}$ is the limit of the sequence $\left\{x_{n}^{(k)}\right\}_{n \in \mathbb{N}}$ constructed from (48) for all $k=1, \ldots, m$.

Proof Since $\mathbf{F}$ is $\left(\preccurlyeq_{I}, \preccurlyeq_{I}\right)$-decreasing and $p$ is an odd integer, we see that $\mathbf{F}^{p}$ is $\left(\preccurlyeq_{I}, \preccurlyeq_{I}\right)$ decreasing. It follows that $\mathbf{x}_{0} \preccurlyeq_{I} \mathbf{x}_{1}$ implies $\mathbf{x}_{1} \succcurlyeq_{I} \mathbf{x}_{2}$, and $\mathbf{x}_{0} \succcurlyeq_{I} \mathbf{x}_{1}$ implies $\mathbf{x}_{1} \preccurlyeq_{I} \mathbf{x}_{2}$. Therefore, we can generate a $\preccurlyeq_{I}$-mixed monotone sequence $\left\{\mathbf{x}_{n}\right\}_{n \in \mathbb{N}}$, which also says that the initial element $\mathbf{x}_{0}$ is a mixed $\preccurlyeq_{I}$-monotone seed element in $X^{m}$. Therefore, the results follow immediately from Theorem 2.16 by taking $\mathbf{f}$ as the identity function. This completes the proof.

Next, we can consider the chain-uniqueness and drop the assumption of continuity of $\mathbf{F}$ by assuming that $(X, d, \preceq)$ preserves the mixed-monotone convergence.

Theorem 4.16 Suppose that the quasi-ordered metric space $(X, d, \preceq)$ is mixed-monotonically complete and preserves the mixed-monotone convergence, and that the metrics $\mathfrak{d}$ and $d$ are compatible in the sense of preserving convergence. Given a disjoint pair I and J of 
$\{1,2, \ldots, m\}$, suppose that there exists $\mathbf{x}_{0} \in X^{m}$ such that the following conditions are satisfied:

- the function $\mathbf{F}:\left(X^{m}, \mathfrak{d}, \preccurlyeq_{I}\right) \rightarrow\left(X^{m}, \mathfrak{d}, \preccurlyeq_{I}\right)$ is $\left(\preccurlyeq_{I}, \preccurlyeq_{I}\right)$-decreasing;

- $\mathbf{x}_{0} \preccurlyeq{ }_{I} \mathbf{F}^{p}\left(\mathbf{x}_{0}\right)$ or $\mathbf{x}_{0} \succcurlyeq_{I} \mathbf{F}^{p}\left(\mathbf{x}_{0}\right)$.

Assume that there exist a function $\rho: X^{m} \times X^{m} \rightarrow \mathbb{R}_{+}$and a function of contractive factor $\varphi:[0, \infty) \rightarrow[0,1)$ such that, for any $\mathbf{x}, \mathbf{y} \in X^{m}$ and any disjoint pair $I^{\circ}$ and $J^{\circ}$ of $\{1, \ldots, m\}$ with $\mathbf{y} \preccurlyeq_{I^{\circ}} \mathbf{x}$ or $\mathbf{x} \preccurlyeq I^{\circ} \mathbf{y}$, the following inequalities

$$
\rho(\mathbf{x}, \mathbf{y}) \leq \sum_{k=1}^{m} d\left(x^{(k)}, y^{(k)}\right)
$$

and

$$
d\left(F_{k}^{p}(\mathbf{x}), F_{k}^{p}(\mathbf{y})\right) \leq \frac{1}{m} \cdot \varphi(\rho(\mathbf{f}(\mathbf{x}), \mathbf{f}(\mathbf{y}))) \cdot \rho(\mathbf{f}(\mathbf{x}), \mathbf{f}(\mathbf{y}))
$$

are satisfied for all $k=1, \ldots, m$ and for some odd integer $p \in \mathbb{N}$. Then the function $\mathbf{F}^{p}$ has a chain-unique fixed point $\hat{\mathbf{x}} \in X^{m}$, where each component $\hat{x}^{(k)}$ of $\hat{\mathbf{x}}$ is the limit of the sequence $\left\{x_{n}^{(k)}\right\}_{n \in \mathbb{N}}$ constructed from (48) for all $k=1, \ldots, m$.

Proof From the proof of Theorem 4.15, we see that the initial element $\mathbf{x}_{0}$ is a mixed $\preccurlyeq_{I}$-monotone seed element in $X^{m}$. Therefore, the results follow immediately from Theorem 2.22 by taking $\mathbf{f}$ as the identity function. This completes the proof.

\section{Fixed points of functions having mixed comparable property in the product spaces}

We shall study the fixed points of functions having mixed comparable property in the product space. Here, we shall consider the mixed-monotonically complete quasi-ordered metric space.

Definition 5.1 Let $I$ and $J$ be a disjoint pair of $\{1,2, \ldots, m\}$. Given a quasi-ordered set $(X, \preceq)$, we consider the corresponding quasi-ordered set $\left(X^{m}, \preccurlyeq_{I}\right)$.

- We say that the function $\mathbf{F}: X^{m} \rightarrow X^{m}$ has the $\preceq$-mixed comparable property if and only if, for any two $\preceq$-mixed comparable elements $\mathbf{x}$ and $\mathbf{y}$ in $X^{m}$, the function values $\mathbf{F}(\mathbf{x})$ and $\mathbf{F}(\mathbf{y})$ in $X^{m}$ are $\preceq$-mixed comparable.

- We say that the function $\mathbf{F}:\left(X^{m}, \preccurlyeq_{I}\right) \rightarrow\left(X^{m}, \preccurlyeq_{I}\right)$ has the $\preccurlyeq_{I}$-comparable property if and only if, for any two elements $\mathbf{x}, \mathbf{y} \in X^{m}$ with $\mathbf{x} \preccurlyeq_{I} \mathbf{y}$ or $\mathbf{y} \preccurlyeq_{I} \mathbf{x}$ (i.e., $\mathbf{x}$ and $\mathbf{y}$ are comparable with respect to ' $\preccurlyeq_{I}$ '), one has either $\mathbf{F}(\mathbf{x}) \preccurlyeq_{I} \mathbf{F}(\mathbf{y})$ or $\mathbf{F}(\mathbf{y}) \preccurlyeq_{I} \mathbf{F}(\mathbf{x})$ (i.e., the function values $\mathbf{F}(\mathbf{x})$ and $\mathbf{F}(\mathbf{y})$ in $X^{m}$ are comparable with respect to ' ${ }_{I}$ ').

It is obvious that if $\mathbf{F}$ is $\left(\preccurlyeq_{I}, \preccurlyeq_{I}\right)$-increasing or $\left(\preccurlyeq_{I}, \preccurlyeq_{I}\right)$-decreasing, then it also has the $\preccurlyeq_{I}$-comparable property.

Theorem 5.2 Suppose that the quasi-ordered metric space $(X, d, \preceq)$ is mixed-monotonically complete, and that the metrics $\mathfrak{d}$ and $d$ are compatible in the sense of preserving continuity. Assume that the function $\mathbf{F}:\left(X^{m}, \mathfrak{d}\right) \rightarrow\left(X^{m}, \mathfrak{d}\right)$ is continuous on $X^{m}$ and has the $\preceq$-mixed comparable property, and that there exist a function $\rho: X^{m} \times X^{m} \rightarrow \mathbb{R}_{+}$and a 
function of contractive factor $\varphi:[0, \infty) \rightarrow[0,1)$ such that, for any two $\preceq$-mixed comparable elements $\mathbf{x}$ and $\mathbf{y}$ in $X^{m}$, the following inequalities

$$
\rho(\mathbf{x}, \mathbf{y}) \leq \sum_{k=1}^{m} d\left(x^{(k)}, y^{(k)}\right)
$$

and

$$
d\left(F_{k}^{p}(\mathbf{x}), F_{k}^{p}(\mathbf{y})\right) \leq \frac{1}{m} \cdot \varphi(\rho(\mathbf{f}(\mathbf{x}), \mathbf{f}(\mathbf{y}))) \cdot \rho(\mathbf{f}(\mathbf{x}), \mathbf{f}(\mathbf{y}))
$$

are satisfied for all $k=1, \ldots, m$ and for some $p \in \mathbb{N}$. If there exists $\mathbf{x}_{0} \in X^{m}$ such that $\mathbf{x}_{0}$ and $\mathbf{F}^{p}\left(\mathbf{x}_{0}\right)$ are $\preceq$-mixed comparable, then $\mathbf{F}^{p}$ has a fixed point $\hat{\mathbf{x}}$ such that each component $\hat{x}^{(k)}$ of $\hat{\mathbf{x}}$ is the limit of the sequence $\left\{x_{n}^{(k)}\right\}_{n \in \mathbb{N}}$ constructed from (48) for all $k=1, \ldots, m$.

Proof According to (48), we see that $\mathbf{x}_{0}$ and $\mathbf{x}_{1}$ are $\preceq$-mixed comparable. Since $\mathbf{F}$ has the $\preceq$-mixed comparable property, we see that $\mathbf{F}^{p}$ has also the $\preceq$-mixed comparable property. It follows that $\mathbf{x}_{1}=\mathbf{F}^{p}\left(\mathbf{x}_{0}\right)$ and $\mathbf{x}_{2}=\mathbf{F}^{p}\left(\mathbf{x}_{1}\right)$ are also $\preceq$-mixed comparable. Therefore, we can generate a mixed $\preceq$-monotone sequence $\left\{\mathbf{x}_{n}\right\}_{n \in \mathbb{N}}$ by observation (d) of Remark 2.4, which also says that the initial element $\mathbf{x}_{0}$ is a mixed $\preceq$-monotone seed element in $X^{m}$. Since $\mathbf{F}$ is continuous on $X^{m}$, it follows that $\mathbf{F}^{p}$ is also continuous on $X^{m}$. Therefore, the result follows from Theorem 2.14 immediately by taking $\mathbf{f}$ as the identity function. This completes the proof.

Next, we can drop the assumption of continuity of $\mathbf{F}$ by assuming that $(X, d, \preceq)$ preserves the mixed-monotone convergence.

Theorem 5.3 Suppose that the quasi-ordered metric space $(X, d, \preceq)$ is mixed-monotonically complete and preserves the mixed-monotone convergence, and that the metrics $\mathfrak{d}$ and $d$ are compatible in the sense of preserving convergence. Assume that the function $\mathbf{F}:\left(X^{m}, \mathfrak{d}\right) \rightarrow\left(X^{m}, \mathfrak{d}\right)$ has the $\preceq$-mixed comparable property, and that there exist a function $\rho: X^{m} \times X^{m} \rightarrow \mathbb{R}_{+}$and a function of contractive factor $\varphi:[0, \infty) \rightarrow[0,1)$ such that, for any two $\preceq$-mixed comparable elements $\mathbf{x}$ and $\mathbf{y}$ in $X^{m}$, the following inequalities

$$
\rho(\mathbf{x}, \mathbf{y}) \leq \sum_{k=1}^{m} d\left(x^{(k)}, y^{(k)}\right)
$$

and

$$
d\left(F_{k}^{p}(\mathbf{x}), F_{k}^{p}(\mathbf{y})\right) \leq \frac{1}{m} \cdot \varphi(\rho(\mathbf{f}(\mathbf{x}), \mathbf{f}(\mathbf{y}))) \cdot \rho(\mathbf{f}(\mathbf{x}), \mathbf{f}(\mathbf{y}))
$$

are satisfied for all $k=1, \ldots, m$ and for some $p \in \mathbb{N}$. Suppose that there exists $\mathbf{x}_{0} \in X^{m}$ such that $\mathbf{x}_{0}$ and $\mathbf{F}^{p}\left(\mathbf{x}_{0}\right)$ are $\preceq$-mixed comparable. Then the following statements hold true.

(i) There exists a unique fixed point $\hat{\mathbf{x}}$ of $\mathbf{F}^{p}$ in the $\preceq$-mixed comparable sense.

(ii) For $p \neq 1$, we further assume that the metrics $\mathfrak{d}$ and $d$ are compatible in the sense of preserving continuity, that the function $\mathbf{F}^{p}$ is continuous on $X^{m}$, and that $\mathbf{F}(\hat{\mathbf{x}})$ and $\hat{\mathbf{x}}$ obtained in (i) are $\preceq$-mixed comparable. Then $\hat{\mathbf{x}}$ is a unique fixed point of $\mathbf{F}$ in the $\preceq$-mixed monotone sense. 
Moreover, each component $\hat{x}^{(k)}$ of $\hat{\mathbf{x}}$ is the limit of the sequence $\left\{x_{n}^{(k)}\right\}_{n \in \mathbb{N}}$ constructed from (48) for all $k=1, \ldots, m$ for all $k=1, \ldots, m$.

Proof According to argument in the proof of Theorem 5.2, we see that the initial element $\mathbf{x}_{0}$ is a mixed $\preceq$-monotone seed element in $X^{m}$. Therefore, part (i) follows from Theorem 2.21 immediately by taking $\mathbf{f}$ as the identity function. Also, part (ii) follows from Theorem 2.26 immediately by taking $\mathbf{f}$ as the identity function. This completes the proof.

Theorem 5.4 Suppose that the quasi-ordered metric space $(X, d, \preceq)$ is mixed-monotonically complete, and that the metrics $\mathfrak{d}$ and $d$ are compatible in the sense of preserving continuity. Given a disjoint pair I and $J$ of $\{1, \ldots, m\}$, assume that the following conditions are satisfied:

- the function $\mathbf{F}:\left(X^{m}, \mathfrak{d}, \preccurlyeq_{I}\right) \rightarrow\left(X^{m}, \mathfrak{d}, \preccurlyeq_{I}\right)$ is continuous on $X^{m}$ and has the $\preccurlyeq_{I}$-comparable property;

- there exists $\mathbf{x}_{0} \in X^{m}$ such that $\mathbf{x}_{0}$ and $\mathbf{F}^{p}\left(\mathbf{x}_{0}\right)$ are comparable with respect to the quasi-order ' $\preccurlyeq$ ' for some $p \in \mathbb{N}$.

Assume that there exist a function $\rho: X^{m} \times X^{m} \rightarrow \mathbb{R}_{+}$and a function of contractive factor $\varphi$ : $[0, \infty) \rightarrow[0,1)$ such that, for any $\mathbf{x}, \mathbf{y} \in X^{m}$ with $\mathbf{y} \preccurlyeq_{I} \mathbf{x}$ or $\mathbf{x} \preccurlyeq_{I} \mathbf{y}$, the following inequalities

$$
\rho(\mathbf{x}, \mathbf{y}) \leq \sum_{k=1}^{m} d\left(x^{(k)}, y^{(k)}\right)
$$

and

$$
d\left(F_{k}^{p}(\mathbf{x}), F_{k}^{p}(\mathbf{y})\right) \leq \frac{1}{m} \cdot \varphi(\rho(\mathbf{f}(\mathbf{x}), \mathbf{f}(\mathbf{y}))) \cdot \rho(\mathbf{f}(\mathbf{x}), \mathbf{f}(\mathbf{y}))
$$

are satisfied for all $k=1, \ldots, m$. Then $\mathbf{F}^{p}$ has a fixed point $\hat{\mathbf{x}}$ such that each component $\hat{x}^{(k)}$ of $\hat{\mathbf{x}}$ is the limit of the sequence $\left\{x_{n}^{(k)}\right\}_{n \in \mathbb{N}}$ constructed from (48) for all $k=1, \ldots, m$.

Proof According to (48), we see that $\mathbf{x}_{0}$ and $\mathbf{x}_{1}$ are comparable with respect to ' $\preccurlyeq{ }_{I}$ '. Since $\mathbf{F}$ has the $\preccurlyeq_{I}$-comparable property, we see that $\mathbf{F}^{p}$ has also the $\preccurlyeq_{I}$-comparable property. It follows that $\mathbf{x}_{1}=\mathbf{F}^{p}\left(\mathbf{x}_{0}\right)$ and $\mathbf{x}_{2}=\mathbf{F}^{p}\left(\mathbf{x}_{1}\right)$ are also comparable with respect to ' $\preccurlyeq I_{I}$. Therefore, we can generate a mixed $\preccurlyeq_{I}$-monotone sequence $\left\{\mathbf{x}_{n}\right\}_{n \in \mathbb{N}}$, which also says that the initial element $\mathbf{x}_{0}$ is a mixed $\preccurlyeq_{I}$-monotone seed element in $X^{m}$. Since $\mathbf{F}$ is continuous on $X^{m}$, it follows that $\mathbf{F}^{p}$ is also continuous on $X^{m}$. Therefore, the result follows from Theorem 2.16 immediately by taking $\mathbf{f}$ as the identity function. This completes the proof.

Next, we can drop the assumption of continuity of $\mathbf{F}$ by assuming that $(X, d, \preceq)$ preserves the mixed-monotone convergence.

Theorem 5.5 Suppose that the quasi-ordered metric space $(X, d, \preceq)$ is mixed-monotonically complete and preserves the mixed-monotone convergence, and that the metrics $\mathfrak{d}$ and $d$ are compatible in the sense of preserving convergence. Given a disjoint pair I and $J$ of $\{1, \ldots, m\}$, assume that the following conditions are satisfied:

- the function $\mathbf{F}:\left(X^{m}, \mathfrak{d}, \preccurlyeq_{I}\right) \rightarrow\left(X^{m}, \mathfrak{d}, \preccurlyeq_{I}\right)$ has the $\preccurlyeq_{I}$-comparable property;

- there exists $\mathbf{x}_{0} \in X^{m}$ such that $\mathbf{x}_{0}$ and $\mathbf{F}^{p}\left(\mathbf{x}_{0}\right)$ are comparable with respect to the quasi-order ' $\preccurlyeq$ ' for some $p \in \mathbb{N}$. 
Suppose that there exist a function $\rho: X^{m} \times X^{m} \rightarrow \mathbb{R}_{+}$and a function of contractive factor $\varphi:[0, \infty) \rightarrow[0,1)$ such that, for any $\mathbf{x}, \mathbf{y} \in X^{m}$ and any disjoint pair $I^{\circ}$ and $J^{\circ}$ of $\{1, \ldots, m\}$ with $\mathbf{y} \preccurlyeq I^{\circ} \mathbf{x}$ or $\mathbf{x} \preccurlyeq I^{\circ} \mathbf{y}$, the following inequalities

$$
\rho(\mathbf{x}, \mathbf{y}) \leq \sum_{k=1}^{m} d\left(x^{(k)}, y^{(k)}\right)
$$

and

$$
d\left(F_{k}^{p}(\mathbf{x}), F_{k}^{p}(\mathbf{y})\right) \leq \frac{1}{m} \cdot \varphi(\rho(\mathbf{f}(\mathbf{x}), \mathbf{f}(\mathbf{y}))) \cdot \rho(\mathbf{f}(\mathbf{x}), \mathbf{f}(\mathbf{y}))
$$

are satisfied for all $k=1, \ldots, m$. Then the following statements hold true.

(i) There exists a chain-unique fixed point $\hat{\mathbf{x}}$ of $\mathbf{F}^{p}$.

(ii) For $p \neq 1$, we further assume that the metrics $\mathfrak{d}$ and $d$ are compatible in the sense of preserving continuity, that the function $\mathbf{F}^{p}$ is continuous on $X^{m}$, and that $\mathbf{F}(\hat{\mathbf{x}})$ and $\hat{\mathbf{x}}$ obtained in (i) are comparable with respect to ' $\preccurlyeq I^{\circ}$ ' for some disjoint pair $I^{\circ}$ and $J^{\circ}$ of $\{1, \ldots, m\}$. Then $\hat{\mathbf{x}}$ is a chain-unique fixed point of $\mathbf{F}$.

Moreover, each component $\hat{x}^{(k)}$ of $\hat{\mathbf{x}}$ is the limit of the sequence $\left\{x_{n}^{(k)}\right\}_{n \in \mathbb{N}}$ constructed from (48) for all $k=1, \ldots, m$.

Proof According to the argument in the proof of Theorem 5.4, we see that the initial element $\mathbf{x}_{0}$ is a mixed $\preccurlyeq_{I}$-monotone seed element in $X^{m}$. Therefore, part (i) follows from Theorem 2.22 immediately by taking $\mathbf{f}$ as the identity function. Also, part (ii) follows from Theorem 2.28 immediately by taking $\mathbf{f}$ as the identity function. This completes the proof.

\section{Applications to the system of integral equations}

Let $\mathcal{C}([0, T], \mathbb{R})$ be the space of all continuous functions from $[0, T]$ into $\mathbb{R}$. We also denote by $\mathcal{C}^{m}([0, T], \mathbb{R})$ the product space of $\mathcal{C}([0, T], \mathbb{R})$ for $m$ times. In the sequel, we shall consider a metric $d$ and a quasi-order ' $\preceq$ ' on $\mathcal{C}([0, T], \mathbb{R})$ such that $(\mathcal{C}([0, T], \mathbb{R}), d, \preceq)$ is monotonically complete or mixed-monotonically complete and preserves the monotone convergence.

Given continuous functions $G:[0, T] \times[0, T] \rightarrow \mathbb{R}_{+}$and $g^{(k)}:[0, T] \times \mathbb{R}^{m} \rightarrow \mathbb{R}$ for $k=$ $1, \ldots, m$, we consider the following system of integral equations:

$$
\int_{0}^{T} G(s, t)\left[g^{(k)}(s, \mathbf{w}(s))+\lambda w^{(k)}(s)\right] d s=w^{(k)}(t)
$$

for $k=1, \ldots, m$, where $\lambda \geq 0$. We shall find $\mathbf{w}^{*} \in \mathcal{C}^{m}([0, T], \mathbb{R})$ such that the system of integral equations (49) are all satisfied, where $w^{(k *)} \in \mathcal{C}([0, T], \mathbb{R})$ is the $k$ th component of $\mathbf{w}^{*}$ for $k=1, \ldots, m$. The solution $\mathbf{w}^{*}$ will be in the sense of chain-uniqueness.

For the vector-valued function $\mathbf{h}:[0, T] \rightarrow \mathbb{R}^{m}$ defined on $[0, T]$, the $k$ th component function of $\mathbf{h}$ is denoted by $h^{(k)}$ for $k=1, \ldots, m$. The integral of $\mathbf{h}$ on $[0, T]$ is defined as the following vector in $\mathbb{R}^{m}$ :

$$
\int_{0}^{T} \mathbf{h}(s) d s=\left(\int_{0}^{T} h^{(1)}(s) d s, \int_{0}^{T} h^{(2)}(s) d s, \ldots, \int_{0}^{T} h^{(m)}(s) d s\right) \in \mathbb{R}^{m} .
$$


Now, we define a vector-valued function $\mathbf{g}:[0, T] \times \mathbb{R}^{m} \rightarrow \mathbb{R}^{m}$ by $\mathbf{g}=\left(g^{(1)}, g^{(2)}, \ldots, g^{(m)}\right)$. Then the system of integral equations as shown in (49) can be written as the following vectorial form of integral equation:

$$
\int_{0}^{T} G(s, t)[\mathbf{g}(s, \mathbf{w}(s))+\lambda \mathbf{w}(s)] d s=\mathbf{w}(t)
$$

where $\lambda \geq 0$. Equivalently, we shall find $\mathbf{w}^{*} \in \mathcal{C}^{m}([0, T], \mathbb{R})$ such that (50) is satisfied, which also says that $\mathbf{w}^{*}$ is a solution of (50).

Definition 6.1 Consider the quasi-ordered metric space $(\mathcal{C}([0, T], \mathbb{R}), d, \preceq)$.

(a) We say that $\mathbf{w}^{*}$ is a unique solution of the system of integral equations (50) in the $\preceq$-mixed comparable sense if and only if the following conditions are satisfied:

- $\mathbf{w}^{*}$ is a solution of (50);

- if $\overline{\mathbf{w}}$ is another solution of (50) such that $\mathbf{w}^{*}$ and $\overline{\mathbf{w}}$ are $\preceq$-mixed comparable, then $\mathbf{w}^{*}=\overline{\mathbf{w}}$.

Given a disjoint pair $I$ and $J$ of $\{1, \ldots, m\}$, consider the product space $\left(\mathcal{C}^{m}([0, T], \mathbb{R}), \mathfrak{d}, \preccurlyeq_{I}\right)$.

(b) We say that $\mathbf{w}^{*}$ is a $\preccurlyeq_{I}$-chain-unique solution of the system of integral equations

(50) if and only if the following conditions are satisfied:

- $\mathbf{w}^{*}$ is a solution of (50);

- if $\overline{\mathbf{w}}$ is another solution of (50) satisfying $\mathbf{w}^{*} \preccurlyeq_{I} \overline{\mathbf{w}}$ or $\overline{\mathbf{w}} \preccurlyeq_{I} \mathbf{w}^{*}$ (i.e., $\mathbf{w}^{*}$ and $\overline{\mathbf{w}}$ are comparable with respect to $\preccurlyeq)$, then $\mathbf{w}^{*}=\overline{\mathbf{w}}$.

Theorem 6.2 Suppose that the quasi-ordered metric space $(\mathcal{C}([0, T], \mathbb{R}), d, \preceq)$ is monotonically complete and preserves the monotone convergence. Let $I$ and $J$ be a disjoint pair of $\{1,2, \ldots, m\}$. Define the function $\mathbf{F}:\left(\mathcal{C}^{m}([0, T], \mathbb{R}), \mathfrak{d}, \preccurlyeq_{I}\right) \rightarrow\left(\mathcal{C}^{m}([0, T], \mathbb{R}), \mathfrak{d}, \preccurlyeq_{I}\right)$ by

$$
\mathbf{F}(\mathbf{w})(t)=\int_{0}^{T} G(s, t)[\mathbf{g}(s, \mathbf{w}(s))+\lambda \mathbf{w}(s)] d s,
$$

where $\mathfrak{d}$ is defined in (5) or (6). Suppose that the following conditions are satisfied:

- $\mathbf{F}$ is $\left(\preccurlyeq_{I}, \preccurlyeq_{I}\right)$-increasing;

- there exist a function $\rho: \mathcal{C}^{m}([0, T], \mathbb{R}) \times \mathcal{C}^{m}([0, T], \mathbb{R}) \rightarrow \mathbb{R}_{+}$and a function of contractive factor $\varphi:[0, \infty) \rightarrow[0,1)$ such that, for any $\mathbf{w}, \overline{\mathbf{w}} \in \mathcal{C}^{m}([0, T], \mathbb{R})$ with $\mathbf{w} \preccurlyeq_{I} \overline{\mathbf{w}}$ or $\overline{\mathbf{w}} \preccurlyeq_{I} \mathbf{w}$, the following inequalities

$$
\rho(\mathbf{w}, \overline{\mathbf{w}}) \leq \sum_{k=1}^{m} d\left(w^{(k)}, \bar{w}^{(k)}\right)
$$

and

$$
d\left(F_{k}(\mathbf{w}), F_{k}(\overline{\mathbf{w}})\right) \leq \frac{1}{m} \cdot \varphi(\rho(\mathbf{w}, \overline{\mathbf{w}})) \cdot \rho(\mathbf{w}, \overline{\mathbf{w}})
$$

are satisfied for all $k=1, \ldots, m$;

- there exists $\mathbf{w}_{0} \in \mathcal{C}^{m}([0, T], \mathbb{R})$ such that $\mathbf{w}_{0} \preccurlyeq_{I} \mathbf{F}\left(\mathbf{w}_{0}\right)$ or $\mathbf{w}_{0} \succcurlyeq_{I} \mathbf{F}\left(\mathbf{w}_{0}\right)$.

Then there exists $a \preccurlyeq_{I}$-chain-unique solution of the system of integral equations (50). 
Proof Since $\mathfrak{d}$ is defined in (5) or (6), we immediately have that the metrics $\mathfrak{d}$ and $d$ are compatible in the sense of preserving convergence. Using condition (a) and considering $p=1$ in Theorem 4.14, we see that $\mathbf{F}$ has $\mathbf{a} \preccurlyeq_{I}$-chain-unique fixed point $\mathbf{w}^{*}$ in $\mathcal{C}^{m}([0, T], \mathbb{R})$. In other words, we have

$$
\int_{0}^{T} G(s, t)\left[\mathbf{g}\left(s, \mathbf{f}\left(\mathbf{w}^{*}(s)\right)\right)+\lambda \mathbf{f}\left(\mathbf{w}^{*}(s)\right)\right] d s=\mathbf{F}\left(\mathbf{w}^{*}\right)=\mathbf{w}^{*},
$$

which says that $\mathbf{w}^{*}$ is a $\preccurlyeq_{I}$-chain-unique solution of the vectorial form of integral equation (50). This completes the proof.

Corollary 6.3 Suppose that the quasi-ordered metric space $(\mathcal{C}([0, T], \mathbb{R}), d, \preceq)$ is monotonically complete and preserves the monotone convergence. Let $I$ and $J$ be a disjoint pair of $\{1,2, \ldots, m\}$. Define the function $\mathbf{F}:\left(\mathcal{C}^{m}([0, T], \mathbb{R}), \mathfrak{d}, \preccurlyeq_{I}\right) \rightarrow\left(\mathcal{C}^{m}([0, T], \mathbb{R}), \mathfrak{d}, \preccurlyeq_{I}\right)$ by

$$
\mathbf{F}(\mathbf{w})(t)=\int_{0}^{T} G(s, t)[\mathbf{g}(s, \mathbf{w}(s))+\lambda \mathbf{w}(s)] d s,
$$

where $\mathfrak{d}$ is defined in (5) or (6). Suppose that the following conditions are satisfied:

- $\mathbf{F}$ is $\left(\preccurlyeq_{I}, \preccurlyeq_{I}\right)$-increasing;

- there exists a function of contractive factor $\varphi:[0, \infty) \rightarrow[0,1)$ such that, for any $\mathbf{w}, \overline{\mathbf{w}} \in \mathcal{C}^{m}([0, T], \mathbb{R})$ with $\mathbf{w} \preccurlyeq_{I} \overline{\mathbf{w}}$ or $\overline{\mathbf{w}} \preccurlyeq_{I} \mathbf{w}$, the following inequality

$$
d\left(F_{k}(\mathbf{w}), F_{k}(\overline{\mathbf{w}})\right) \leq \varphi\left(m \cdot\left(\min _{k=1, \ldots, m} d\left(w^{(k)}, \bar{w}^{(k)}\right)\right)\right) \cdot\left(\min _{k=1, \ldots, m} d\left(w^{(k)}, \bar{w}^{(k)}\right)\right)
$$

is satisfied for all $k=1, \ldots, m$;

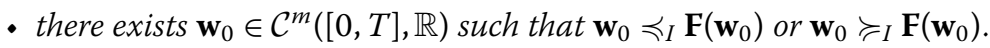

Then there exists $a \preccurlyeq_{I}$-chain-unique solution of the system of integral equations (50).

Proof For $\mathbf{a}, \mathbf{b} \in \mathcal{C}^{m}([0, T], \mathbb{R})$, we define the function $\rho: \mathcal{C}^{m}([0, T], \mathbb{R}) \times \mathcal{C}^{m}([0, T], \mathbb{R}) \rightarrow$ $\mathbb{R}_{+}$by

$$
\rho(\mathbf{a}, \mathbf{b})=m \cdot\left(\min _{k=1, \ldots, m} d\left(a^{(k)}, b^{(k)}\right)\right) \leq \sum_{k=1}^{m} d\left(a^{(k)}, b^{(k)}\right) .
$$

Then the desired result follows from Theorem 6.2 immediately.

Remark 6.4 We have the following observations.

- The assumptions for inequalities (51) and (52) are really weak, since we just assume that they are satisfied for $\preccurlyeq_{I}$-comparable elements. In other words, if $\mathbf{x}$ and $\mathbf{y}$ are not $\preccurlyeq I_{I}$-comparable, we do not need to check inequalities (51) and (52).

- In Theorem 6.2, according to Remark 4.7, if the function $\mathbf{F}$ is assumed to have the $I$-mixed monotone property on $\mathcal{C}^{m}([0, T], \mathbb{R})$ instead of assuming it to be $\left(\preccurlyeq_{I}, \preccurlyeq_{I}\right.$ )-increasing, then the results are still valid.

Lemma 6.5 For any $a, b \in \mathcal{C}([0, T], \mathbb{R})$, we define

$a \preceq^{*} b \quad$ if and only if $a(s) \leq b(s)$ for all $s \in[0, T]$. 
Then the quasi-ordered metric space $\left(\mathcal{C}([0, T], \mathbb{R}), d, \preceq^{*}\right)$ preserves the monotone convergence.

Proof Let $\left\{a_{n}\right\}_{n \in \mathbb{N}}$ be a $\preceq$-increasing sequence in $(\mathcal{C}([0, T], \mathbb{R}), d, \preceq)$, and let $\hat{a}$ be the $d$-limit of $\left\{a_{n}\right\}_{n \in \mathbb{N}}$. Suppose that there exists $n_{1} \in \mathbb{N}$ such that $a_{n_{1}} \npreceq \hat{a}$; that is, there exists $s_{0} \in$ $[0, T]$ such that $a_{n_{1}}\left(s_{0}\right)>\hat{a}\left(s_{0}\right)$. Since $a_{n}(s) \leq a_{n+1}(s)$ for all $s \in[0, T]$ and $n \in \mathbb{N}$, it follows that $a_{n+1}\left(s_{0}\right) \geq a_{n}\left(s_{0}\right)>\hat{a}\left(s_{0}\right)$ for all $n \geq n_{1}$, which contradicts the convergence $a_{n}\left(s_{0}\right) \rightarrow$ $\hat{a}\left(s_{0}\right)$. Therefore, we must have $a_{n}(s) \leq \hat{a}(s)$ for all $s \in[0, T]$, i.e., $a_{n} \preceq \hat{a}$. If $\left\{a_{n}\right\}_{n \in \mathbb{N}}$ is a $\preceq$-decreasing sequence in $(\mathcal{C}([0, T], \mathbb{R}), d, \preceq)$ and converges to $\hat{a}$, then we can similarly show that $a_{n} \succeq \hat{a}$ for all $n \in \mathbb{N}$. This completes the proof.

The following result is well known.

Lemma 6.6 For any $a, b \in \mathcal{C}([0, T], \mathbb{R})$, we define

$$
d^{*}(a, b)=\sup _{s \in[0, T]}|a(s)-b(s)|
$$

Then the quasi-ordered metric space $\left(\mathcal{C}([0, T], \mathbb{R}), d^{*}, \preceq\right)$ is complete.

Given a disjoint pair $I$ and $J$ of $\{1,2, \ldots, m\}$, we can consider a quasi-ordered set $\left(\mathbb{R}^{m}, \preccurlyeq_{I}^{(m)}\right)$ that depends on $I$, where, for any $\mathbf{x}, \mathbf{y} \in \mathbb{R}^{m}$,

$$
\mathbf{x} \preccurlyeq_{I}^{(m)} \mathbf{y} \quad \text { if and only if } \quad x^{(k)} \leq y^{(k)} \quad \text { for } k \in I \quad \text { and } \quad y^{(k)} \leq x^{(k)} \quad \text { for } k \in J \text {. }
$$

Then we have the following interesting existence.

Theorem 6.7 Let $\left(\mathcal{C}([0, T], \mathbb{R}), d^{*}, \preceq^{*}\right)$ be a quasi-ordered metric space with the metric $d^{*}$ and the quasi-order $\preceq^{*}$ defined in (54) and (53), respectively. Let I and J be a disjoint pair of $\{1,2, \ldots, m\}$. Define the function $\mathbf{F}:\left(\mathcal{C}^{m}([0, T], \mathbb{R}), \mathfrak{d}^{*}, \preccurlyeq_{I}^{*}\right) \rightarrow\left(\mathcal{C}^{m}([0, T], \mathbb{R}), \mathfrak{d}^{*}, \preccurlyeq_{I}^{*}\right)$ by

$$
\mathbf{F}(\mathbf{w})(t)=\int_{0}^{T} G(s, t)[\mathbf{g}(s, \mathbf{w}(s))+\lambda \mathbf{w}(s)] d s,
$$

where $\preccurlyeq_{I}^{*}$ is defined in (1) according to $\preceq^{*}$, and $\mathfrak{d}^{*}$ is defined in (5) or (6) according to $d^{*}$. Suppose that the following conditions are satisfied:

- $\mathbf{F}$ is $\left(\preccurlyeq_{I}^{*}, \preccurlyeq_{I}^{*}\right)$-increasing;

- there exists a function $\rho: \mathcal{C}^{m}([0, T], \mathbb{R}) \times \mathcal{C}^{m}([0, T], \mathbb{R}) \rightarrow \mathbb{R}_{+}$such that, for any $\mathbf{a}, \mathbf{b} \in \mathcal{C}^{m}([0, T], \mathbb{R})$ with $\mathbf{a} \preccurlyeq_{I}^{*} \mathbf{b}$ or $\mathbf{b} \preccurlyeq_{I}^{*} \mathbf{a}$, the following inequality

$$
\rho(\mathbf{a}, \mathbf{b}) \leq \sum_{k=1}^{m} d^{*}\left(a^{(k)}, b^{(k)}\right)
$$

is satisfied;

- there exists a function of contractive factor $\varphi:[0, \infty) \rightarrow[0,1)$ such that, for any $\mathbf{x}, \mathbf{y} \in \mathbb{R}^{m}$ with $\mathbf{x} \preccurlyeq_{I}^{(m)} \mathbf{y}$ or $\mathbf{y} \preccurlyeq_{I}^{(m)} \mathbf{x}$, the following inequality

$$
\left|g^{(k)}(s, \mathbf{x})+\lambda x^{(k)}-g^{(k)}(s, \mathbf{y})-\lambda y^{(k)}\right| \leq \bar{\phi}(\mathbf{x}, \mathbf{y}) \cdot \hat{\phi}(\mathbf{x}, \mathbf{y})
$$


is satisfied for $k=1, \ldots, m$, where $\lambda \geq 0$, and the functions $\bar{\phi}: \mathbb{R}^{2 m} \rightarrow \mathbb{R}_{+}$and $\hat{\phi}: \mathbb{R}^{2 m} \rightarrow \mathbb{R}_{+}$satisfy the following inequalities: for $\mathbf{a}, \mathbf{b} \in \mathcal{C}^{m}([0, T], \mathbb{R})$,

$$
\hat{\phi}(\mathbf{a}(s), \mathbf{b}(s)) \leq \rho(\mathbf{a}, \mathbf{b}) \quad \text { for all } s \in[0, T]
$$

and

$$
\sup _{t \in[0, T]} \int_{0}^{T} G(s, t) \cdot \bar{\phi}(\mathbf{a}(s), \mathbf{b}(s)) d s \leq \frac{1}{m} \cdot \varphi(\rho(\mathbf{a}, \mathbf{b}))
$$

- there exists $\mathbf{w}_{0} \in \mathcal{C}^{m}([0, T], \mathbb{R})$ such that $\mathbf{w}_{0} \preccurlyeq_{I}^{*} \mathbf{F}\left(\mathbf{w}_{0}\right)$ or $\mathbf{F}\left(\mathbf{w}_{0}\right) \preccurlyeq_{I}^{*} \mathbf{w}_{0}$.

Then there exists $a \preccurlyeq_{I}^{*}$-chain-unique solution of the system of integral equations (50).

Proof Lemmas 6.5 and 6.6 say that the quasi-ordered metric space $\left(\mathcal{C}([0, T], \mathbb{R}), d^{*}, \preceq^{*}\right)$ is complete and preserves the monotone convergence. For $\mathbf{w} \preccurlyeq_{I}^{*} \overline{\mathbf{w}}$ or $\overline{\mathbf{w}} \preccurlyeq_{I}^{*} \mathbf{w}$, it means, for each $s \in[0, T]$,

$$
w^{(k)}(s) \leq \bar{w}^{(k)}(s) \quad \text { for } k \in I \quad \text { and } \quad \bar{w}^{(k)}(s) \leq w^{(k)}(s) \quad \text { for } k \in J
$$

or

$$
w^{(k)}(s) \leq \bar{w}^{(k)}(s) \quad \text { for } k \in I \quad \text { and } \quad \bar{w}^{(k)}(s) \leq w^{(k)}(s) \quad \text { for } k \in J
$$

which also says that

$$
\mathbf{w}(s) \preccurlyeq_{I}^{(m)} \overline{\mathbf{w}}(s) \quad \text { or } \quad \overline{\mathbf{w}}(s) \preccurlyeq_{I}^{(m)} \mathbf{w}(s) \quad \text { for each } s \in[0, T] .
$$

Then we have

$$
\begin{aligned}
& d\left(F_{k}(\mathbf{w}), F_{k}(\overline{\mathbf{w}})\right) \\
& \quad=\sup _{t \in[0, T]}\left|F_{k}(\mathbf{w})(t)-F_{k}(\overline{\mathbf{w}})(t)\right| \\
& \quad=\sup _{t \in[0, T]} \int_{0}^{T} G(s, t) \cdot\left|g^{(k)}(s, \mathbf{w}(s))+\lambda w^{(k)}(s)-g^{(k)}(s, \overline{\mathbf{w}}(s))-\lambda \bar{w}^{(k)}(s)\right| d s \\
& \quad \leq \sup _{t \in[0, T]} \int_{0}^{T} G(s, t) \cdot \bar{\phi}(\mathbf{w}(s), \overline{\mathbf{w}}(s)) \cdot \hat{\phi}(\mathbf{w}(s), \overline{\mathbf{w}}(s)) d s \quad \text { by (55) and (58)) } \\
& \leq \sup _{t \in[0, T]} \int_{0}^{T} G(s, t) \cdot \bar{\phi}(\mathbf{w}(s), \overline{\mathbf{w}}(s)) \cdot \rho(\mathbf{w}, \overline{\mathbf{w}}) d s \quad(\text { by }(56)) \\
& \quad \leq \frac{1}{m} \cdot \varphi(\rho(\mathbf{w}, \overline{\mathbf{w}})) \cdot \rho(\mathbf{w}, \overline{\mathbf{w}}) \quad(\text { by }(57)) .
\end{aligned}
$$

Using Theorem 6.2, we complete the proof.

Corollary 6.8 Let $\left(\mathcal{C}([0, T], \mathbb{R}), d^{*}, \preceq^{*}\right)$ be a quasi-ordered metric space with the metric $d^{*}$ and the quasi-order $\preceq^{*}$ defined in (54) and (53), respectively. Let I and J be a disjoint pair 
of $\{1,2, \ldots, m\}$. Define the function $\mathbf{F}:\left(\mathcal{C}^{m}([0, T], \mathbb{R}), \mathfrak{d}^{*}, \preccurlyeq_{I}^{*}\right) \rightarrow\left(\mathcal{C}^{m}([0, T], \mathbb{R}), \mathfrak{d}^{*}, \preccurlyeq_{I}^{*}\right)$ by

$$
\mathbf{F}(\mathbf{w})(t)=\int_{0}^{T} G(s, t)[\mathbf{g}(s, \mathbf{w}(s))+\lambda \mathbf{w}(s)] d s
$$

where $\preccurlyeq_{I}^{*}$ is defined in (1) according to $\preceq^{*}$, and $\mathfrak{d}^{*}$ is defined in (5) or (6) according to $d^{*}$. Suppose that the following conditions are satisfied:

- $\mathbf{F}$ is $\left(\preccurlyeq_{I}^{*}, \preccurlyeq_{I}^{*}\right)$-increasing;

- there exists a function of contractive factor $\varphi:[0, \infty) \rightarrow[0,1)$ such that, for any $\mathbf{x}, \mathbf{y} \in \mathbb{R}^{m}$ with $\mathbf{x} \preccurlyeq_{I}^{(m)} \mathbf{y}$ or $\mathbf{y} \preccurlyeq_{I}^{(m)} \mathbf{x}$, the following inequality

$$
\left|g^{(k)}(s, \mathbf{x})+\lambda x^{(k)}-g^{(k)}(s, \mathbf{y})-\lambda y^{(k)}\right| \leq m \cdot\left(\min _{k=1, \ldots, m}\left|x^{(k)}-y^{(k)}\right|\right) \cdot \bar{\phi}(\mathbf{x}, \mathbf{y})
$$

is satisfied for $k=1, \ldots, m$, where $\lambda \geq 0$, and the function $\bar{\phi}: \mathbb{R}^{2 m} \rightarrow \mathbb{R}_{+}$satisfies the following inequality

$$
\sup _{t \in[0, T]} \int_{0}^{T} G(s, t) \cdot \bar{\phi}(\mathbf{a}, \mathbf{b}) d s \leq \frac{1}{m} \cdot \varphi\left(m \cdot\left(\min _{k=1, \ldots, m} d^{*}\left(a^{(k)}, b^{(k)}\right)\right)\right)
$$

- there exists $\mathbf{w}_{0} \in \mathcal{C}^{m}([0, T], \mathbb{R})$ such that $\mathbf{w}_{0} \preccurlyeq_{I}^{*} \mathbf{F}\left(\mathbf{w}_{0}\right)$ or $\mathbf{F}\left(\mathbf{w}_{0}\right) \preccurlyeq_{I}^{*} \mathbf{w}_{0}$.

Then there exists $a \preccurlyeq_{I}^{*}$-chain-unique solution of the system of integral equations (50).

Proof For $\mathbf{a}, \mathbf{b} \in \mathcal{C}^{m}([0, T], \mathbb{R})$, we define the function $\rho: \mathcal{C}^{m}([0, T], \mathbb{R}) \times \mathcal{C}^{m}([0, T], \mathbb{R}) \rightarrow$ $\mathbb{R}_{+}$by

$$
\rho(\mathbf{a}, \mathbf{b})=m \cdot\left(\min _{k=1, \ldots, m} d^{*}\left(a^{(k)}, b^{(k)}\right)\right) \leq \sum_{k=1}^{m} d^{*}\left(a^{(k)}, b^{(k)}\right)
$$

and the function $\hat{\phi}: \mathbb{R}^{2 m} \rightarrow \mathbb{R}_{+}$by

$$
\hat{\phi}(\mathbf{x}, \mathbf{y})=m \cdot\left(\min _{k=1, \ldots, m}\left|x^{(k)}-y^{(k)}\right|\right) .
$$

Since

$$
\left|a^{(k)}(s)-b^{(k)}(s)\right| \leq d^{*}\left(a^{(k)}, b^{(k)}\right) \quad \text { for all } s \in[0, T],
$$

we have

$$
\hat{\phi}(\mathbf{a}(s), \mathbf{b}(s))=m \cdot\left(\min _{k=1, \ldots, m}\left|a^{(k)}(s)-b^{(k)}(s)\right|\right) \leq m \cdot\left(\min _{k=1, \ldots, m} d^{*}\left(a^{(k)}, b^{(k)}\right)\right)=\rho(\mathbf{a}, \mathbf{b})
$$

for $s \in[0, T]$. The desired result follows from Theorem 6.7 immediately, and the proof is complete.

Compared to Corollary 6.8, we consider a different type of inequalities below.

Theorem 6.9 Let $\left(\mathcal{C}([0, T], \mathbb{R}), d^{*}, \preceq^{*}\right)$ be a quasi-ordered metric space with the metric $d^{*}$ and the quasi-order $\preceq^{*}$ defined in (54) and (53), respectively. Let I and J be a disjoint pair 
of $\{1,2, \ldots, m\}$. Define the function $\mathbf{F}:\left(\mathcal{C}^{m}([0, T], \mathbb{R}), \mathfrak{d}^{*}, \preccurlyeq_{I}^{*}\right) \rightarrow\left(\mathcal{C}^{m}([0, T], \mathbb{R}), \mathfrak{d}^{*}, \preccurlyeq_{I}^{*}\right)$ by

$$
\mathbf{F}(\mathbf{w})(t)=\int_{0}^{T} G(s, t)[\mathbf{g}(s, \mathbf{w}(s))+\lambda \mathbf{w}(s)] d s
$$

where $\preccurlyeq_{I}^{*}$ is defined in (1) according to $\preceq^{*}$, and $\mathfrak{d}^{*}$ is defined in (5) or (6) according to $d^{*}$. Suppose that the following conditions are satisfied:

- $\mathbf{F}$ is $\left(\preccurlyeq_{I}^{*}, \preccurlyeq_{I}^{*}\right)$-increasing;

- there exists a function of contractive factor $\varphi:[0, \infty) \rightarrow[0,1)$ such that, for any $\mathbf{x}, \mathbf{y} \in \mathbb{R}^{m}$ with $\mathbf{x} \preccurlyeq_{I}^{(m)} \mathbf{y}$ or $\mathbf{y} \preccurlyeq_{I}^{(m)} \mathbf{x}$, the following inequality

$$
\left|g^{(k)}(s, \mathbf{x})+\lambda x^{(k)}-g^{(k)}(s, \mathbf{y})-\lambda y^{(k)}\right| \leq m \cdot\left(\min _{k=1, \ldots, m}\left|x^{(k)}-y^{(k)}\right|\right) \cdot \bar{\phi}^{*}\left(x^{(k)}, y^{(k)}\right)
$$

is satisfied for $k=1, \ldots, m$, where $\lambda \geq 0$, and the function $\bar{\phi}^{*}: \mathbb{R}^{2} \rightarrow \mathbb{R}_{+}$satisfies the following inequality: for $\mathbf{a}, \mathbf{b} \in \mathcal{C}^{m}([0, T], \mathbb{R})$,

$$
\sup _{t \in[0, T]} \int_{0}^{T} G(s, t) \cdot \bar{\phi}^{*}\left(a^{(k)}(s), b^{(k)}(s)\right) d s \leq \frac{1}{m} \cdot \varphi\left(m \cdot\left(\min _{k=1, \ldots, m} d^{*}\left(a^{(k)}, b^{(k)}\right)\right)\right)
$$

for $k=1, \ldots, m$;

- there exists $\mathbf{w}_{0} \in \mathcal{C}^{m}([0, T], \mathbb{R})$ such that $\mathbf{w}_{0} \preccurlyeq_{I}^{*} \mathbf{F}\left(\mathbf{w}_{0}\right)$ or $\mathbf{F}\left(\mathbf{w}_{0}\right) \preccurlyeq_{I}^{*} \mathbf{w}_{0}$.

Then there exists $a \preccurlyeq_{I}^{*}$-chain-unique solution of the system of integral equations (50).

Proof For $\mathbf{a}, \mathbf{b} \in \mathcal{C}^{m}([0, T], \mathbb{R})$, we define a function $\rho: \mathcal{C}^{m}([0, T], \mathbb{R}) \times \mathcal{C}^{m}([0, T], \mathbb{R}) \rightarrow \mathbb{R}_{+}$ by (59). Now, we have

$$
\begin{aligned}
& d\left(F_{k}(\mathbf{w}), F_{k}(\overline{\mathbf{w}})\right) \\
& \quad=\sup _{t \in[0, T]}\left|F_{k}(\mathbf{w})(t)-F_{k}(\overline{\mathbf{w}})(t)\right| \\
& \quad=\sup _{t \in[0, T]} \int_{0}^{T} G(s, t) \cdot\left|g^{(k)}(s, \mathbf{w}(s))+\lambda w^{(k)}(s)-g^{(k)}(s, \overline{\mathbf{w}}(s))-\lambda \bar{w}^{(k)}(s)\right| d s \\
& \quad \leq \sup _{t \in[0, T]} \int_{0}^{T} G(s, t) \cdot \bar{\phi}^{*}\left(w^{(k)}(s), \bar{w}^{(k)}(s)\right) \cdot m \cdot\left(\min _{k=1, \ldots, m}\left|w^{(k)}(s)-\bar{w}^{(k)}(s)\right|\right) d s
\end{aligned}
$$

(by (58) and (61))

$$
\begin{aligned}
& \left.\leq \sup _{t \in[0, T]} \int_{0}^{T} G(s, t) \cdot \bar{\phi}^{*}\left(w^{(k)}(s), \bar{w}^{(k)}(s)\right) \cdot \rho(\mathbf{w}, \overline{\mathbf{w}}) d s \quad \text { (by }(60)\right) \\
& \leq \frac{1}{m} \cdot \varphi(\rho(\mathbf{w}, \overline{\mathbf{w}})) \cdot \rho(\mathbf{w}, \overline{\mathbf{w}}) \quad(\text { by }(62) \text { and }(59)) .
\end{aligned}
$$

Using Theorem 6.2, we complete the proof.

\section{Applications to the system of ordinary differential equations}

We consider the quasi-ordered metric space $(\mathcal{C}([0, T], \mathbb{R}), d, \preceq)$ and the following system of ordinary differential equations:

$$
\left\{\begin{array}{l}
w_{k}^{\prime}(t)=g^{(k)}(t, \mathbf{w}(t)) \quad \text { for } t \in[0, T], \\
w_{k}(0)=w_{k}(T)
\end{array} \quad \text { for } k=1, \ldots, m,\right.
$$


where $T>0, w_{k} \in \mathcal{C}([0, T], \mathbb{R})$ and $g^{(k)}:[0, T] \times \mathbb{R}^{m} \rightarrow \mathbb{R}$ are continuous functions for $k=1, \ldots, m$.

Definition 7.1 Consider the quasi-ordered metric space $(\mathcal{C}([0, T], \mathbb{R}), d, \preceq)$ and the product space $\mathcal{C}^{m}([0, T], \mathbb{R})$.

(a) We say that $\mathbf{w}^{*}$ is a unique solution of the system of boundary value problem (63) in the $\preceq$-mixed comparable sense if and only if the following conditions are satisfied:

- $\mathbf{w}^{*}$ is a solution of (63);

- if $\overline{\mathbf{w}}$ is another solution of (63) such that $\mathbf{w}^{*}$ and $\overline{\mathbf{w}}$ are $\preceq$-mixed comparable, then $\mathbf{w}^{*}=\overline{\mathbf{w}}$.

Let $I$ and $J$ be the disjoint pair of $\{1, \ldots, m\}$.

(b) We say that $\mathbf{w}^{*}$ is a $\preccurlyeq_{I}$-chain-unique solution of the system of boundary value problem (63) if and only if the following conditions are satisfied:

- $\mathbf{w}^{*}$ is a solution of (63);

- if $\overline{\mathbf{w}}$ is another solution of (63) satisfying $\mathbf{w}^{*} \preccurlyeq_{I} \overline{\mathbf{w}}$ or $\overline{\mathbf{w}} \preccurlyeq_{I} \mathbf{w}^{*}$ (i.e., $\mathbf{w}^{*}$ and $\overline{\mathbf{w}}$ are comparable with respect to $\preccurlyeq)$, then $\mathbf{w}^{*}=\overline{\mathbf{w}}$.

Definition 7.2 Let $(\mathcal{C}([0, T], \mathbb{R}), \preceq)$ be a quasi-ordered set.

- We say that the quasi-order ' $\leq$ ' is compatible with the addition if and only if, for any $a, b \in \mathcal{C}([0, T], \mathbb{R})$ with $a \preceq b$, we have $a+c \preceq b+c$ for any $c \in \mathcal{C}([0, T], \mathbb{R})$.

- We say that the quasi-order ' $\preceq$ ' is compatible with the nonnegative multiplication if and only if, for any $a, b \in \mathcal{C}([0, T], \mathbb{R})$ with $a \preceq b$, we have $a c \preceq b c$ for any nonnegative function $c \in \mathcal{C}([0, T], \mathbb{R})$.

Remark 7.3 Suppose that the quasi-order ' $\preceq$ ' is compatible with the addition. If $a_{1} \preceq b_{1}$ and $a_{2} \preceq b_{2}$, we want to claim $a_{1}+a_{2} \preceq b_{1}+b_{2}$. By definition, we immediately have

$$
a_{1}+a_{2} \preceq b_{1}+a_{2} \text { and } b_{1}+a_{2} \preceq b_{1}+b_{2} \text {. }
$$

The transitivity proves the desired claim.

Remark 7.4 Suppose that the quasi-order ' $\preceq$ ' in $\mathcal{C}([0, T], \mathbb{R})$ is compatible with the addition and nonnegative multiplication. Then we have the following observations.

- For $\mathbf{w}_{1}, \mathbf{w}_{2}, \overline{\mathbf{w}}_{1}, \overline{\mathbf{w}}_{2} \in \mathcal{C}^{m}([0, T], \mathbb{R})$. If $\mathbf{w}_{1} \preccurlyeq{ }_{I} \mathbf{w}_{2}$, and $\overline{\mathbf{w}}_{1} \preccurlyeq{ }_{I} \overline{\mathbf{w}}_{2}$, then, from Remark 7.3, we have $\mathbf{w}_{1}+\overline{\mathbf{w}}_{1} \preccurlyeq{ }_{I} \mathbf{w}_{2}+\overline{\mathbf{w}}_{2}$.

- If $\mathbf{w}_{1}, \mathbf{w}_{2} \in \mathcal{C}^{m}([0, T], \mathbb{R})$ with $\mathbf{w}_{1} \preccurlyeq \preccurlyeq_{I} \mathbf{w}_{2}$, then $c \cdot \mathbf{w}_{1} \preccurlyeq{ }_{I} c \cdot \mathbf{w}_{2}$ for any nonnegative function $c \in \mathcal{C}([0, T], \mathbb{R})$ by the compatibility for nonnegative function.

Consider a disjoint pair $I$ and $J$ of $\{1,2, \ldots, m\}$. We say that the function $\mathbf{g}:[0, T] \times \mathbb{R}^{m} \rightarrow$ $\mathbb{R}^{m}$ defined by

$$
\mathbf{g}=\left(g^{(1)}, \ldots, g^{(m)}\right)
$$

is $\left(\preccurlyeq_{I}, \preccurlyeq_{I}\right)$-increasing if and only if $\mathbf{w}_{1}, \mathbf{w}_{2} \in \mathcal{C}^{m}([0, T], \mathbb{R})$ with $\mathbf{w}_{1} \preccurlyeq_{I} \mathbf{w}_{2}$ implies $\mathbf{g}\left(\cdot, \mathbf{w}_{1}\right) \preccurlyeq_{I}$ $\mathbf{g}\left(\cdot, \mathbf{w}_{2}\right)$. By referring to Definition 4.4 , we can similarly define the other kinds of monotonic concepts. 
Definition 7.5 Let $(\mathcal{C}([0, T], \mathbb{R}), \preceq)$ be a quasi-ordered set. Consider the function $\mathcal{G}$ : $[0, T] \times[0, T] \rightarrow \mathbb{R}$ such that $\mathcal{G}(s, \cdot) \in \mathcal{C}([0, T], \mathbb{R})$ for any fixed $s \in[0, T]$ and $\mathcal{G}(\cdot, t) \in$ $\mathcal{C}([0, T], \mathbb{R})$ for any fixed $t \in[0, T]$. We say that the integral of function $\mathcal{G}$ is compatible with the quasi-order ' $\preceq$ ' if and only if, for any fixed $t \in[0, T], \mathcal{G}_{1}(\cdot, t) \preceq \mathcal{G}_{2}(\cdot, t)$ implies

$$
\int_{0}^{T} \mathcal{G}_{1}(s, t) d s \preceq \int_{0}^{T} \mathcal{G}_{2}(s, t) d s .
$$

Example 7.6 For any $a, b \in \mathcal{C}([0, T], \mathbb{R})$, we define

$$
a \preceq b \quad \text { if and only if } \quad a(t) \leq b(t) \text { for all } t \in[0, T]
$$

If the function $\mathcal{G}:[0, T] \times[0, T] \rightarrow \mathbb{R}$ satisfies $\mathcal{G}(s, \cdot) \in \mathcal{C}([0, T], \mathbb{R})$ for any fixed $s \in[0, T]$ and $\mathcal{G}(\cdot, t) \in \mathcal{C}([0, T], \mathbb{R})$ for any fixed $t \in[0, T]$, then the integral of function $\mathcal{G}$ is compatible with the quasi-order ' $\preceq$ '. Indeed, for any fixed $t \in[0, T], \mathcal{G}_{1}(\cdot, t) \preceq \mathcal{G}_{2}(\cdot, t)$ means that $\mathcal{G}_{1}(s, t) \leq \mathcal{G}_{2}(s, t)$ for all $s \in[0, T]$ by (65), which also says that, for any $t \in[0, T]$,

$$
\int_{0}^{T} \mathcal{G}_{1}(s, t) d s \leq \int_{0}^{T} \mathcal{G}_{2}(s, t) d s .
$$

This shows (64) by (65) again.

Theorem 7.7 Suppose that the quasi-ordered metric space $(\mathcal{C}([0, T], \mathbb{R}), d, \preceq)$ is monotonically complete and preserves the monotone convergence. Let $I$ and $J$ be a disjoint pair of $\{1,2, \ldots, m\}$, and let $\lambda>0$. Define the function $G:[0, T] \times[0, T] \rightarrow \mathbb{R}_{+}$by

$$
G(s, t)= \begin{cases}\frac{e^{\lambda(T+s-t)}}{e^{\lambda T}-1} & \text { if } 0 \leq s<t \leq T, \\ \frac{e^{\lambda(s-t)}}{e^{\lambda T}-1} & \text { if } 0 \leq t<s \leq T,\end{cases}
$$

and the function $\mathbf{F}:\left(\mathcal{C}^{m}([0, T], \mathbb{R}), \mathfrak{d}, \preccurlyeq_{I}\right) \rightarrow\left(\mathcal{C}^{m}([0, T], \mathbb{R}), \mathfrak{d}, \preccurlyeq_{I}\right)$ by

$$
\mathbf{F}(\mathbf{w})(t)=\int_{0}^{T} G(s, t)[\mathbf{g}(s, \mathbf{w}(s))+\lambda \mathbf{w}(s)] d s,
$$

where $\mathfrak{d}$ is defined in (5) or (6). Suppose that the following conditions are satisfied:

- the quasi-order ' $\leq$ ' in $\mathcal{C}([0, T], \mathbb{R})$ is compatible with the addition and nonnegative multiplication;

- $\mathbf{g}$ is $\left(\preccurlyeq_{I}, \preccurlyeq I\right)$-increasing;

- for any $\mathbf{w} \in \mathcal{C}^{m}([0, T], \mathbb{R})$, the integrals of the following functions

$$
\mathcal{G}_{k}(s, t)=G(s, t)\left[g^{(k)}(s, \mathbf{w}(s))+\lambda w_{k}(s)\right]
$$

are compatible with the quasi-order ' $\leq$ ' for $k=1, \ldots, m$.

- there exist a function $\rho: \mathcal{C}^{m}([0, T], \mathbb{R}) \times \mathcal{C}^{m}([0, T], \mathbb{R}) \rightarrow \mathbb{R}_{+}$and a function of contractive factor $\varphi:[0, \infty) \rightarrow[0,1)$ such that, for any $\mathbf{w}, \overline{\mathbf{w}} \in \mathcal{C}^{m}([0, T], \mathbb{R})$ with $\mathbf{w} \preccurlyeq_{I} \overline{\mathbf{w}}$ or $\overline{\mathbf{w}} \preccurlyeq_{I} \mathbf{w}$, the following inequalities

$$
\rho(\mathbf{w}, \overline{\mathbf{w}}) \leq \sum_{k=1}^{m} d\left(w^{(k)}, \bar{w}^{(k)}\right)
$$


and

$$
d\left(F_{k}(\mathbf{w}), F_{k}(\overline{\mathbf{w}})\right) \leq \frac{1}{m} \cdot \varphi(\rho(\mathbf{w}, \overline{\mathbf{w}})) \cdot \rho(\mathbf{w}, \overline{\mathbf{w}})
$$

are satisfied for all $k=1, \ldots, m$;

- there exists $\mathbf{w}_{0} \in \mathcal{C}^{m}([0, T], \mathbb{R})$ such that $\mathbf{w}_{0} \preccurlyeq{ }_{I} \mathbf{F}\left(\mathbf{w}_{0}\right)$ or $\mathbf{w}_{0} \succcurlyeq_{I} \mathbf{F}\left(\mathbf{w}_{0}\right)$.

Then the system of boundary value problem (63) has a $\preccurlyeq_{I}$-chain-unique solution.

Proof First of all, problem (63) can be written as follows:

$$
\left\{\begin{array}{l}
w_{k}^{\prime}(t)+\lambda w_{k}(t)=g^{(k)}(t, \mathbf{w}(t))+\lambda w_{k}(t) \quad \text { for } t \in[0, T], \\
w_{k}(0)=w_{k}(T)
\end{array} \text { for } k=1, \ldots, m .\right.
$$

We shall rewrite problem (68) as a system of integral equations. Multiplying $e^{\lambda t}$ on both sides, we have

$$
e^{\lambda t} \cdot w_{k}^{\prime}(t)+e^{\lambda t} \cdot \lambda w_{k}(t)=\left[g^{(k)}(t, \mathbf{w}(t))+\lambda w_{k}(t)\right] \cdot e^{\lambda t},
$$

which is equivalent to

$$
\left(e^{\lambda t} \cdot w_{k}(t)\right)^{\prime}=\left[g^{(k)}(t, \mathbf{w}(t))+\lambda w_{k}(t)\right] \cdot e^{\lambda t} \quad \text { for } t \in[0, T] .
$$

By taking integration on both sides, we have

$$
e^{\lambda t} \cdot w_{k}(t)=w_{k}(0)+\int_{0}^{t}\left[g^{(k)}(s, \mathbf{w}(s))+\lambda w_{k}(s)\right] \cdot e^{\lambda s} d s \quad \text { for } t \in[0, T] .
$$

Since $w_{k}(0)=w_{k}(T)$, we obtain

$$
e^{\lambda T} \cdot w_{k}(0)=e^{\lambda T} \cdot w_{k}(T)=w_{k}(0)+\int_{0}^{T}\left[g^{(k)}(s, \mathbf{w}(s))+\lambda w_{k}(s)\right] \cdot e^{\lambda s} d s,
$$

which implies

$$
w_{k}(0)=\int_{0}^{T} \frac{e^{\lambda s}}{e^{\lambda T}-1} \cdot\left[g^{(k)}(s, \mathbf{w}(s))+\lambda w_{k}(s)\right] d s .
$$

From (69) and (70), for $t \in[0, T]$, we have

$$
\begin{aligned}
e^{\lambda t} \cdot w_{k}(t)= & \int_{0}^{T} \frac{e^{\lambda s}}{e^{\lambda T}-1} \cdot\left[g^{(k)}(s, \mathbf{w}(s))+\lambda w_{k}(s)\right] d s \\
& +\int_{0}^{t}\left[g^{(k)}(s, \mathbf{w}(s))+\lambda w_{k}(s)\right] \cdot e^{\lambda s} d s \\
= & \int_{0}^{t} \frac{e^{\lambda(T+s)}}{e^{\lambda T}-1} \cdot\left[g^{(k)}(s, \mathbf{w}(s))+\lambda w_{k}(s)\right] d s \\
& +\int_{t}^{T} \frac{e^{\lambda s}}{e^{\lambda T}-1} \cdot\left[g^{(k)}(s, \mathbf{w}(s))+\lambda w_{k}(s)\right] d s .
\end{aligned}
$$


Therefore, we obtain the following integral equations:

$$
w_{k}(t)=\int_{0}^{T} G(s, t)\left[g^{(k)}(s, \mathbf{w}(s))+\lambda w_{k}(s)\right] d s \quad \text { for } k=1, \ldots, m,
$$

where $G(s, t)$ is given in (66). We see that if $\mathbf{w}^{*}$ is a solution of integral equation (71), then $\mathbf{w}^{*}$ is a solution of problem (68), which also says that $\mathbf{w}^{*}$ is a solution of the original boundary value problem (63).

Since $G(s, t) \geq 0$, the quasi-order ' $\preceq$ ' is compatible with the addition and nonnegative multiplication, and $\mathbf{g}$ is $\left(\preccurlyeq_{I}, \preccurlyeq_{I}\right)$-increasing, it follows that, for each fixed $t \in[0, T]$, the function $G(s, t) \cdot[\mathbf{g}(s, \mathbf{w}(s))+\lambda \mathbf{w}(s)]$ is $\left(\preccurlyeq_{I}, \preccurlyeq I\right)$-increasing by Remark 7.4. Since the integrals of the functions $\mathcal{G}_{k}$ defined in (67) are compatible with the quasi-order ' $\preceq$ ' for $k=1, \ldots, m$, it shows that the function $\mathbf{F}$ is $\left(\preccurlyeq_{I}, \preccurlyeq_{I}\right)$-increasing. Theorem 6.2 says that the system of

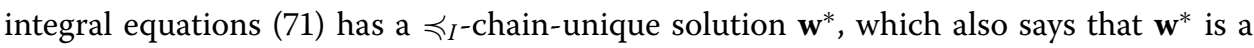
solution of the original boundary value problem (63). However, this $\preccurlyeq_{I}$-chain-uniqueness is about the system of integral equations (71), which is not the $\preccurlyeq{ }_{I}$-chain-uniqueness about the original boundary value problem (63). Now, let $\hat{\mathbf{w}}$ be another solution of the system of boundary value problem (63) such that $\hat{\mathbf{w}}$ and $\mathbf{w}^{*}$ are comparable with respect to ' $\preccurlyeq I_{I}$. By referring to the derivation of (71), we can show that $\hat{\mathbf{w}}$ is also a solution of the system of integral equations (71). By the $\preccurlyeq_{I}$-chain-uniqueness given in Theorem 6.2 , it follows that $\mathbf{w}^{*}=\hat{\mathbf{w}}$; that is, $\mathbf{w}^{*}$ is a $\preccurlyeq{ }_{I}$-chain-unique solution of the system of boundary value problem (63). This completes the proof.

Corollary 7.8 Suppose that the quasi-ordered metric space $(\mathcal{C}([0, T], \mathbb{R}), d, \preceq)$ is monotonically complete and preserves the monotone convergence. Let $I$ and $J$ be a disjoint pair of $\{1,2, \ldots, m\}$, and let $\lambda>0$. Define the function $G:[0, T] \times[0, T] \rightarrow \mathbb{R}_{+}$by

$$
G(s, t)= \begin{cases}\frac{e^{\lambda(T+s-t)}}{e^{\lambda T}-1} & \text { if } 0 \leq s<t \leq T, \\ \frac{e^{\lambda(s-t)}}{e^{\lambda T}-1} & \text { if } 0 \leq t<s \leq T,\end{cases}
$$

and the function $\mathbf{F}:\left(\mathcal{C}^{m}([0, T], \mathbb{R}), \mathfrak{d}, \preccurlyeq_{I}\right) \rightarrow\left(\mathcal{C}^{m}([0, T], \mathbb{R}), \mathfrak{d}, \preccurlyeq_{I}\right)$ by

$$
\mathbf{F}(\mathbf{w})(t)=\int_{0}^{T} G(s, t)[\mathbf{g}(s, \mathbf{w}(s))+\lambda \mathbf{w}(s)] d s,
$$

where $\mathfrak{d}$ is defined in (5) or (6). Suppose that the following conditions are satisfied:

- the quasi-order ' $\preceq$ ' in $\mathcal{C}([0, T], \mathbb{R})$ is compatible with the addition and nonnegative multiplication;

- $\mathbf{g}$ is $\left(\preccurlyeq_{I}, \preccurlyeq_{I}\right)$-increasing;

- for any $\mathbf{w} \in \mathcal{C}^{m}([0, T], \mathbb{R})$, the integrals of the following functions

$$
\mathcal{G}_{k}(s, t)=G(s, t)\left[g^{(k)}(s, \mathbf{w}(s))+\lambda w_{k}(s)\right]
$$

are compatible with the quasi-order '

- there exists a function of contractive factor $\varphi:[0, \infty) \rightarrow[0,1)$ such that, for any $\mathbf{w}, \overline{\mathbf{w}} \in \mathcal{C}^{m}([0, T], \mathbb{R})$ with $\mathbf{w} \preccurlyeq_{I} \overline{\mathbf{w}}$ or $\overline{\mathbf{w}} \preccurlyeq_{I} \mathbf{w}$, the following inequality

$$
d\left(F_{k}(\mathbf{w}), F_{k}(\overline{\mathbf{w}})\right) \leq \varphi\left(m \cdot\left(\min _{k=1, \ldots, m} d\left(w^{(k)}, \bar{w}^{(k)}\right)\right)\right) \cdot\left(\min _{k=1, \ldots, m} d\left(w^{(k)}, \bar{w}^{(k)}\right)\right)
$$

is satisfied for all $k=1, \ldots, m$; 
- there exists $\mathbf{w}_{0} \in \mathcal{C}^{m}([0, T], \mathbb{R})$ such that $\mathbf{w}_{0} \preccurlyeq{ }_{I} \mathbf{F}\left(\mathbf{w}_{0}\right)$ or $\mathbf{w}_{0} \succcurlyeq_{I} \mathbf{F}\left(\mathbf{w}_{0}\right)$.

Then the system of boundary value problem (63) has $a \preccurlyeq_{I}$-chain-unique solution.

Proof For $\mathbf{a}, \mathbf{b} \in \mathcal{C}^{m}([0, T], \mathbb{R})$, we define the function $\rho: \mathcal{C}^{m}([0, T], \mathbb{R}) \times \mathcal{C}^{m}([0, T], \mathbb{R}) \rightarrow$ $\mathbb{R}_{+}$by

$$
\rho(\mathbf{a}, \mathbf{b})=m \cdot\left(\min _{k=1, \ldots, m} d\left(a^{(k)}, b^{(k)}\right)\right) \leq \sum_{k=1}^{m} d\left(a^{(k)}, b^{(k)}\right)
$$

Then the desired result follows from Theorem 7.7 immediately.

Theorem 7.9 Let $\left(\mathcal{C}([0, T], \mathbb{R}), d^{*}, \preceq^{*}\right)$ be a quasi-ordered metric space with the metric $d^{*}$ and the quasi-order ${ }^{*}$ defined in (54) and (53), respectively. Let I and J be a disjoint pair of $\{1,2, \ldots, m\}$, and let $\preccurlyeq_{I}^{*}$ be defined in (1) according to $\preceq^{*}$. Let $\lambda>0$. Define the function $G:[0, T] \times[0, T] \rightarrow \mathbb{R}_{+}$by

$$
G(s, t)= \begin{cases}\frac{e^{\lambda(T+s-t)}}{e^{\lambda T}-1} & \text { if } 0 \leq s<t \leq T, \\ \frac{e^{\lambda(s-t)}}{e^{\lambda T}-1} & \text { if } 0 \leq t<s \leq T .\end{cases}
$$

Suppose that the following conditions are satisfied:

- there exists a function $\rho: \mathcal{C}([0, T], \mathbb{R}) \times \mathcal{C}([0, T], \mathbb{R}) \rightarrow \mathbb{R}_{+}$such that, for any $\mathbf{a}, \mathbf{b} \in \mathcal{C}^{m}([0, T], \mathbb{R})$ with $\mathbf{a} \preccurlyeq_{I}^{*} \mathbf{b}$ or $\mathbf{b} \preccurlyeq_{I}^{*} \mathbf{a}$, the following inequality

$$
\rho(\mathbf{a}, \mathbf{b}) \leq \sum_{k=1}^{m} d^{*}\left(a^{(k)}, b^{(k)}\right)
$$

is satisfied;

- there exists $\mu>0$ with $\mu<\lambda$ such that, for any $\mathbf{x}, \mathbf{y} \in \mathbb{R}^{m}$ with $\mathbf{x} \preccurlyeq_{I}^{(m)} \mathbf{y}$ or $\mathbf{y} \preccurlyeq_{I}^{(m)} \mathbf{x}$, the following inequality

$$
\left|g^{(k)}(s, \mathbf{x})+\lambda x^{(k)}-g^{(k)}(s, \mathbf{y})-\lambda y^{(k)}\right| \leq \frac{\mu}{m} \cdot \hat{\phi}(\mathbf{x}, \mathbf{y})
$$

is satisfied for $k=1, \ldots, m$, where the function $\hat{\phi}: \mathbb{R}^{2 m} \rightarrow \mathbb{R}_{+}$satisfies the following inequality: for $\mathbf{a}, \mathbf{b} \in \mathcal{C}^{m}([0, T], \mathbb{R})$,

$$
\hat{\phi}(\mathbf{a}(s), \mathbf{b}(s)) \leq \rho(\mathbf{a}, \mathbf{b}) \quad \text { for all } s \in[0, T]
$$

- there exists $\overline{\mathbf{w}} \in \mathcal{C}^{m}([0, T], \mathbb{R})$ such that

$$
\overline{\mathbf{w}} \preccurlyeq_{I}^{*} \int_{0}^{T} G(s, t)[\mathbf{g}(s, \mathbf{w}(s))+\lambda \mathbf{w}(s)] d s
$$

or

$$
\int_{0}^{T} G(s, t)[\mathbf{g}(s, \mathbf{w}(s))+\lambda \mathbf{w}(s)] d s \preccurlyeq_{I}^{*} \overline{\mathbf{w}} .
$$

Then the system of boundary value problem (63) has $a \preccurlyeq_{I}$-chain-unique solution. 
Proof It is easy to see that the quasi-order ' $\complement^{*}$ ' in $\mathcal{C}([0, T], \mathbb{R})$ is compatible with the addition and nonnegative multiplication. It follows that $\mathbf{g}$ is $\left(\preccurlyeq_{I}^{*}, \preccurlyeq_{I}^{*}\right)$-increasing. According to Example 7.6, for any $\mathbf{w} \in \mathcal{C}^{m}([0, T], \mathbb{R})$, we see that the integrals of the following functions

$$
\mathcal{G}_{k}(s, t)=G(s, t)\left[g^{(k)}(s, \mathbf{w}(s))+\lambda w_{k}(s)\right]
$$

are compatible with the quasi-order ' $\preceq$ ' for $k=1, \ldots, m$. Now, we define

$$
\mathbf{F}(\mathbf{w})(t)=\int_{0}^{T} G(s, t)[\mathbf{g}(s, \mathbf{w}(s))+\lambda \mathbf{w}(s)] d s
$$

In order to obtain

$$
d\left(F_{k}(\mathbf{w}), F_{k}(\overline{\mathbf{w}})\right) \leq \frac{1}{m} \cdot \varphi(\rho(\mathbf{w}, \overline{\mathbf{w}})) \cdot \rho(\mathbf{w}, \overline{\mathbf{w}}),
$$

from the proof of Theorem 6.7, we need to check inequalities (55) and (57). Now, we take $\bar{\phi}$ as a constant function with value $\mu / m$, and $\varphi$ as a constant function with value $\mu / \lambda<1$. Then $\varphi$ is a function of contractive factor. According to (74), we see that inequality (55) is satisfied. We also have

$$
\begin{aligned}
& \sup _{t \in[0, T]} \int_{0}^{T} G(s, t) \bar{\phi}(\mathbf{a}, \mathbf{b}) d s \\
& \quad=\frac{\mu}{m} \cdot \sup _{t \in[0, T]} \int_{0}^{T} G(s, t) d s \\
& \quad=\frac{\mu}{m} \cdot \sup _{t \in[0, T]}\left[\frac{1}{e^{\lambda T}-1} \cdot\left(\left.\frac{1}{\lambda} \cdot e^{\lambda(T+s-t)}\right|_{0} ^{t}+\left.\frac{1}{\lambda} \cdot e^{\lambda(s-t)}\right|_{t} ^{T}\right)\right] \quad(\text { by }(73)) \\
& \quad=\frac{\mu}{m} \cdot \frac{1}{e^{\lambda T}-1} \cdot \frac{1}{\lambda} \cdot\left(e^{\lambda T}-1\right) \\
& \quad=\frac{1}{m} \cdot \frac{\mu}{\lambda}=\frac{1}{m} \cdot \varphi(\rho(\mathbf{a}, \mathbf{b})),
\end{aligned}
$$

which shows that inequality (57) is satisfied. According to the proof of Theorem 6.7, we see that (75) is satisfied. Therefore, the desired result follows from Theorem 7.7, and the proof is complete.

Definition 7.10 For $\mathbf{w} \in \mathcal{C}^{m}([0, T], \mathbb{R})$, we define some solution concepts as follows.

- We say that $\mathbf{w}$ is a sub-solution of problem (63) if and only if

$$
\left\{\begin{array}{l}
w_{k}^{\prime}(t) \leq g^{(k)}(t, \mathbf{w}(t)), \\
w_{k}(0)=w_{k}(T)
\end{array} \quad \text { for } k=1, \ldots, m .\right.
$$

- We say that $\mathbf{w}$ is a sup-solution of problem (63) if and only if

$$
\left\{\begin{array}{l}
w_{k}^{\prime}(t) \geq g^{(k)}(t, \mathbf{w}(t)) \quad \text { for } t \in[0, T], \\
w_{k}(0)=w_{k}(T)
\end{array} \quad \text { for } k=1, \ldots, m .\right.
$$


- Let $I$ and $J$ be a disjoint pair of $\{1,2, \ldots, m\}$. We say that $\mathbf{w}$ is an $(I, J)$-mixed solution of problem (63) if and only if

$$
\left\{\begin{array}{l}
w_{k}^{\prime}(t) \leq g^{(k)}(t, \mathbf{w}(t)) \quad \text { for } k \in I \text { and } t \in[0, T] \\
w_{k}^{\prime}(t) \geq g^{(k)}(t, \mathbf{w}(t)) \quad \text { for } k \in J \text { and } t \in[0, T] \\
w_{k}(0)=w_{k}(T) \quad \text { for } k=1, \ldots, m,
\end{array}\right.
$$

where $I$ is allowed to be a nonempty set.

We have to emphasize that the $(I, J)$-mixed solution and $(J, I)$-mixed solution are essentially the same. It is obvious that if $I=\emptyset$, then the $(I, J)$-mixed solution is also a supsolution, and if $J=\emptyset$, then the $(I, J)$-mixed solution is also a sub-solution.

Theorem 7.11 Let $\left(\mathcal{C}([0, T], \mathbb{R}), d^{*}, \preceq^{*}\right)$ be a quasi-ordered metric space with the metric $d^{*}$ and the quasi-order $\preceq^{*}$ defined in (54) and (53), respectively. Let I and $J$ be a disjoint pair of $\{1,2, \ldots, m\}$, and let $\preccurlyeq_{I}^{*}$ be defined in (1) according to $\preceq^{*}$. Suppose that the following conditions are satisfied:

- there exists a function $\rho: \mathcal{C}([0, T], \mathbb{R}) \times \mathcal{C}([0, T], \mathbb{R}) \rightarrow \mathbb{R}_{+}$such that, for any $\mathbf{a}, \mathbf{b} \in \mathcal{C}^{m}([0, T], \mathbb{R})$ with $\mathbf{a} \preccurlyeq_{I}^{*} \mathbf{b}$ or $\mathbf{b} \preccurlyeq_{I}^{*} \mathbf{a}$, the following inequality

$$
\rho(\mathbf{a}, \mathbf{b}) \leq \sum_{k=1}^{m} d^{*}\left(a^{(k)}, b^{(k)}\right)
$$

is satisfied;

- there exists $\mu>0$ with $\mu<\lambda$ such that, for any $\mathbf{x}, \mathbf{y} \in \mathbb{R}^{m}$ with $\mathbf{x} \preccurlyeq_{I}^{(m)} \mathbf{y}$ or $\mathbf{y} \preccurlyeq_{I}^{(m)} \mathbf{x}$, the following inequality

$$
\left|g^{(k)}(s, \mathbf{x})+\lambda x^{(k)}-g^{(k)}(s, \mathbf{y})-\lambda y^{(k)}\right| \leq \frac{\mu}{m} \cdot \hat{\phi}(\mathbf{x}, \mathbf{y})
$$

is satisfied for $k=1, \ldots, m$, where the function $\hat{\phi}: \mathbb{R}^{2 m} \rightarrow \mathbb{R}_{+}$satisfies the following inequality: for $\mathbf{a}, \mathbf{b} \in \mathcal{C}^{m}([0, T], \mathbb{R})$,

$$
\hat{\phi}(\mathbf{a}(s), \mathbf{b}(s)) \leq \rho(\mathbf{a}, \mathbf{b}) \quad \text { for all } s \in[0, T]
$$

- there exists an (I,J)-mixed solution of problem (63).

Then the system of boundary value problem (63) has a $\preccurlyeq_{I}$-chain-unique solution.

Proof Let $\overline{\mathbf{w}}$ be an $(I, J)$-mixed solution of problem (63). Then, for $k \in I$, we have

$$
\left\{\begin{array}{l}
\bar{w}_{k}^{\prime}(t) \leq g^{(k)}(t, \overline{\mathbf{w}}(t)) \quad \text { for } t \in[0, T], \\
\bar{w}_{k}(0)=\bar{w}_{k}(T),
\end{array}\right.
$$

which implies

$$
\left\{\begin{array}{l}
\bar{w}_{k}^{\prime}(t)+\lambda \bar{w}_{k}(t) \leq g^{(k)}(t, \overline{\mathbf{w}}(t))+\lambda \bar{w}_{k}(t) \quad \text { for } t \in[0, T], \\
\bar{w}_{k}(0)=\bar{w}_{k}(T) .
\end{array}\right.
$$


Multiplying $e^{\lambda t}$ on both sides, we have

$$
e^{\lambda t} \cdot \bar{w}_{k}^{\prime}(t)+e^{\lambda t} \cdot \lambda \bar{w}_{k}(t) \leq\left[g^{(k)}(t, \overline{\mathbf{w}}(t))+\lambda \bar{w}_{k}(t)\right] \cdot e^{\lambda t},
$$

which is equivalent to

$$
\left(e^{\lambda t} \cdot \bar{w}_{k}(t)\right)^{\prime} \leq\left[g^{(k)}(t, \overline{\mathbf{w}}(t))+\lambda \bar{w}_{k}(t)\right] \cdot e^{\lambda t} \quad \text { for } t \in[0, T] .
$$

By taking integration on both sides, we have

$$
e^{\lambda t} \cdot \bar{w}_{k}(t) \leq \bar{w}_{k}(0)+\int_{0}^{t}\left[g^{(k)}(s, \overline{\mathbf{w}}(s))+\lambda \bar{w}_{k}(s)\right] \cdot e^{\lambda s} d s \quad \text { for } t \in[0, T] .
$$

Since $\bar{w}_{k}(0)=\bar{w}_{k}(T)$, we obtain

$$
e^{\lambda T} \cdot \bar{w}_{k}(0) \leq e^{\lambda T} \cdot \bar{w}_{k}(T)=\bar{w}_{k}(0)+\int_{0}^{T}\left[g^{(k)}(s, \overline{\mathbf{w}}(s))+\lambda \bar{w}_{k}(s)\right] \cdot e^{\lambda s} d s,
$$

which implies

$$
\bar{w}_{k}(0) \leq \int_{0}^{T} \frac{e^{\lambda s}}{e^{\lambda T}-1} \cdot\left[g^{(k)}(s, \overline{\mathbf{w}}(s))+\lambda \bar{w}_{k}(s)\right] d s .
$$

From (76) and (77), for $t \in[0, T]$, we have

$$
\begin{aligned}
e^{\lambda t} \cdot \bar{w}_{k}(t) \leq & \int_{0}^{T} \frac{e^{\lambda s}}{e^{\lambda T}-1} \cdot\left[g^{(k)}(s, \overline{\mathbf{w}}(s))+\lambda \bar{w}_{k}(s)\right] d s \\
& +\int_{0}^{t}\left[g^{(k)}(s, \overline{\mathbf{w}}(s))+\lambda \bar{w}_{k}(s)\right] \cdot e^{\lambda s} d s \\
= & \int_{0}^{t} \frac{e^{\lambda(T+s)}}{e^{\lambda T}-1} \cdot\left[g^{(k)}(s, \overline{\mathbf{w}}(s))+\lambda \bar{w}_{k}(s)\right] d s \\
& +\int_{t}^{T} \frac{e^{\lambda s}}{e^{\lambda T}-1} \cdot\left[g^{(k)}(s, \overline{\mathbf{w}}(s))+\lambda \bar{w}_{k}(s)\right] d s .
\end{aligned}
$$

Therefore, we obtain

$$
\bar{w}_{k}(t) \leq \int_{0}^{T} G(s, t)\left[g^{(k)}(s, \overline{\mathbf{w}}(s))+\lambda \bar{w}_{k}(s)\right] d s \quad \text { for } k \in I,
$$

where $G(s, t)$ is given in $(66)$. We can similarly show that

$$
\bar{w}_{k}(t) \geq \int_{0}^{T} G(s, t)\left[g^{(k)}(s, \overline{\mathbf{w}}(s))+\lambda \bar{w}_{k}(s)\right] d s \quad \text { for } k \in J,
$$

which says that

$$
\overline{\mathbf{w}} \preccurlyeq_{I}^{*} \int_{0}^{T} G(s, t)[\mathbf{g}(s, \overline{\mathbf{w}}(s))+\lambda \overline{\mathbf{w}}(s)] d s .
$$

Therefore, the desired result follows from Theorem 7.9 immediately. 
The assumption for the existence of $(I, J)$-mixed solution in Theorem 7.11 can be replaced by the assumption for the existence of sub-solution or sup-solution in which $J$ or $I$ is taken to be an empty set, respectively.

\section{Competing interests}

The author declares that he has no competing interests.

\section{Acknowledgements}

The author was supported by the Ministry of Science and Technology in Taiwan.

\section{Received: 4 October 2014 Accepted: 9 December 2014 Published: 23 Dec 2014}

\section{References}

1. Choudhury, BS, Kundu, A: A coupled coincidence point result in partially ordered metric spaces for compatible mappings. Nonlinear Anal. 73, 2524-2531 (2010)

2. Latif, A, Tweddle, I: Some results on coincidence points. Bull. Aust. Math. Soc. 59, 111-117 (1999)

3. Tarafdar, E, Watson, PJ: A coincidence point theorem and related results. Appl. Math. Lett. 11, 37-40 (1998)

4. Väth, M: Coincidence points of function pairs based on compactness properties. Glasg. Math. J. 44, $209-230$ (2002)

5. Abbas, M, Khan, AR, Nazir, T: Coupled common fixed point results in two generalized metric spaces. Appl. Math. Comput. 217, 6328-6336 (2011)

6. Ahmed, MA: Common fixed points of hybrid maps and an application. Comput. Math. Appl. 60, 1888-1894 (2010)

7. Bhatt, A, Chandra, H, Sahu, DR: Common fixed point theorems for occasionally weakly compatible mappings under relaxed conditions. Nonlinear Anal. 73, 176-182 (2010)

8. Chang, S-S, Lee, HWJ, Chan, CK: A new hybrid method for solving a generalized equilibrium problem, solving a variational inequality problem and obtaining common fixed points in Banach spaces, with applications. Nonlinear Anal. 73, 2260-2270 (2010)

9. Ćirić, L: Common fixed point theorems for a family of non-self mappings in convex metric spaces. Nonlinear Anal. 71 , 1662-1669 (2009)

10. Ćirić, L, Samet, B, Vetro, C: Common fixed point theorems for families of occasionally weakly compatible mappings. Math. Comput. Model. 53, 631-636 (2011)

11. Ćirić, L, Ume, JS: Some common fixed point theorems for weakly compatible mappings. J. Math. Anal. Appl. 314, 488-499 (2006)

12. Colao, V, Marino, G: Strong convergence for a minimization problem on points of equilibrium and common fixed points of an infinite family of nonexpansive mappings. Nonlinear Anal. 73, 3513-3524 (2010)

13. Liu, Q-Y, Liu, Z-B, Huang, N-J: Approximating the common fixed points of two sequences of uniformly quasi-Lipschitzian mappings in convex metric spaces. Appl. Math. Comput. 216, 883-889 (2010)

14. Moradi, S, Fathi, Z, Analouee, E: The common fixed point of single-valued generalized $\varphi_{f}$-weakly contractive mappings. Appl. Math. Lett. 24, 771-776 (2011)

15. Qin, X, Cho, SY, Zhou, H: Common fixed points of a pair of non-expansive mappings with applications to convex feasibility problems. Glasg. Math. J. 52, 241-252 (2010)

16. Mizoguchi, N, Takahashi, W: Fixed point theorems for multivalued mappings on complete metric spaces. J. Math. Anal. Appl. 141, 177-188 (1989)

17. Takahashi, W: Fixed point theorems for new nonlinear mappings in a Hilbert space. J. Nonlinear Convex Anal. 11, 79-88 (2010)

18. Nadler, SB Jr:: Multi-valued contractive mappings. Pac. J. Math. 30, 475-488 (1969)

19. Suzuki, T: Mizoguchi-Takahashi's fixed point theorem is a real generalization of Nadler's. J. Math. Anal. Appl. 340, $752-755(2008)$

10.1186/1029-242X-2014-518

Cite this article as: Wu: Coincidence point and common fixed point theorems in the product spaces of mixed-monotonically complete quasi-ordered metric spaces and their applications to the systems of integral equations and ordinary differential equations. Journal of Inequalities and Applications 2014, 2014:518 